\title{
EXISTENCE AND REGULARITY OF OPTIMAL SHAPES FOR ELLIPTIC OPERATORS WITH DRIFT
}

\author{
EMMANUEL RUSS, BAPTISTE TREY, BOZHIDAR VELICHKOV
}

\begin{abstract}
This paper is dedicated to the study of shape optimization problems for the first eigenvalue of the elliptic operator with drift $L=-\Delta+V(x) \cdot \nabla$ with Dirichlet boundary conditions, where $V$ is a bounded vector field. In the first instance, we prove the existence of a principal eigenvalue $\lambda_{1}(\Omega, V)$ for a bounded quasi-open set $\Omega$ which enjoys similar properties to the case of open sets. Then, given $m>0$ and $\tau \geq 0$, we show that the minimum of the following non-variational problem

$$
\min \left\{\lambda_{1}(\Omega, V): \Omega \subset D \text { quasi-open, }|\Omega| \leq m,\|V\|_{L^{\infty}} \leq \tau\right\} .
$$

is achieved, where the box $D \subset \mathbb{R}^{d}$ is a bounded open set. The existence when $V$ is fixed, as well as when $V$ varies among all the vector fields which are the gradient of a Lipschitz function, are also proved.

The second interest and main result of this paper is the regularity of the optimal shape $\Omega^{*}$ solving the minimization problem

$$
\min \left\{\lambda_{1}(\Omega, \nabla \Phi): \Omega \subset D \text { quasi-open, }|\Omega| \leq m\right\},
$$

where $\Phi$ is a given Lipschitz function on $D$. We prove that the optimal set $\Omega^{*}$ is open and that its topological boundary $\partial \Omega^{*}$ is composed of a regular part, which is locally the graph of a $C^{1, \alpha}$ function, and a singular part, which is empty if $d<d^{*}$, discrete if $d=d^{*}$ and of locally finite $\mathcal{H}^{d-d^{*}}$ Hausdorff measure if $d>d^{*}$, where $d^{*} \in\{5,6,7\}$ is the smallest dimension at which there exists a global solution to the one-phase free boundary problem with singularities. Moreover, if $D$ is smooth, we prove that, for each $x \in \partial \Omega^{*} \cap \partial D, \partial \Omega^{*}$ is $C^{1,1 / 2}$ in a neighborhood of $x$.
\end{abstract}

\section{Contents}

1. Introduction and main results

Optimal shapes for a fixed vector field

Outline of the proof and plan of the paper

2. Preliminaries

2.1. Capacity, quasi-open sets and quasi-continuous functions

2.2. PDEs on quasi-open sets

2.3. The $\gamma$-convergence and the weak- $\gamma$-convergence 9

3. The principal eigenvalue on quasi-open sets 11

4. Existence of optimal domains 15

4.1. Optimal drifts on a fixed domain 15

4.2. Shape optimization problem over domains and vector fields $\quad 16$

5. Regularity of the optimal sets $\quad 18$

5.1. Boundedness of the eigenfunctions $\quad 19$

5.2. Pointwise definition of the solutions 20

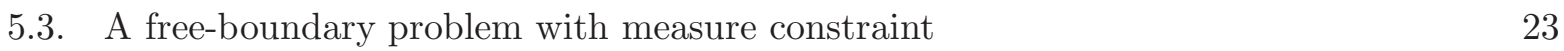

5.4. An internal variation optimality condition $\quad 24$

5.5. Almost optimality of $u$ at small scales $\quad 28$

5.6. Lipschitz continuity of the eigenfunctions on the optimal sets

Date: May 17, 2019.

1991 Mathematics Subject Classification. 49Q10, 35R35, 47A75.

Key words and phrases. shape optimization, operators with drift, principal eigenvalue, $\gamma$-convergence, quasiopen sets, regularity of the free boundaries. 
5.7. Non-degeneracy of the eigenfunctions and finiteness of the perimeter of $\Omega_{u}$

5.8. Blow-up sequences and blow-up limits $\quad 36$

5.9. Regularity of the free boundary 39

5.10. Monotonicity formula and some further estimates on the dimension of the singular set

Appendix A. Extremality conditions and Lebesgue density $\quad 44$

A.1. Reduction to the case $\lambda=0 \quad 45$

A.2. Proof of Proposition A.1 in the case $\lambda=0 \quad 46$

References 48

\section{INTRODUCTION AND MAIN RESULTS}

Let $D$ be a bounded connected open set in $\mathbb{R}^{d}, d \geq 2$. For any bounded vector field $V: D \rightarrow \mathbb{R}^{d}$ and any connected open set $\Omega \subset D$, we consider the elliptic operator with drift $L=-\Delta+V(x) \cdot \nabla$. In this paper we study variational optimization problems in which the variables are both the domain $\Omega$ and the drift $V$, and the cost functional is defined through the operator $L$. The aim of the present paper is twofold. From one side, we develop an existence theory for shape optimization problems for operators with drift. On the other hand, we study the regularity of the optimal shapes for vector fields $V$ that are gradients of potentials $\Phi: D \rightarrow \mathbb{R}$. We focus on the model problem

$$
\min \left\{\lambda_{1}(\Omega, V): \Omega \subset D,|\Omega| \leq m,\|V\|_{L^{\infty}} \leq \tau\right\},
$$

where $m>0$ and $\tau \geq 0$ are fixed constants, and $\lambda_{1}(\Omega, V)$ is the principal eigenvalue of the operator $L$. Our main results are the following.

Theorem 1.1. Let $D \subset \mathbb{R}^{d}$ be a bounded open set, and $0<m<|D|$ and $\tau \geq 0$ be fixed constants. Then, there exist a quasi-open set $\Omega \subset D$ and a vector field $V: D \rightarrow \mathbb{R}^{\bar{d}}$ such that the couple $(\Omega, V)$ is a solution to the shape optimization problem (1.1).

In particular, we prove in Theorem 3.3 below, that the principal eigenvalue $\lambda_{1}(\Omega, V)$ of the (nonself-adjoint) operator $L$ is well-defined on any quasi-open set $\Omega \subset D$. Preciesly, we will show that for any quasi-open set $\Omega$, there is a real eigenvalue $\lambda_{1}(\Omega, V)$ of the operator $L$ such that $\lambda_{1}(\Omega, V) \leq \operatorname{Re} \lambda$, for any other eigenvalue $\lambda \in \mathbb{C}$ of $L$.

Theorem 1.2. Let $D \subset \mathbb{R}^{d}$ be a bounded open set, and $0<m<|D|$ and $\tau \geq 0$ be fixed constants. Then the shape optimization problem

$$
\min \left\{\lambda_{1}(\Omega, \nabla \Phi): \Omega \subset D \text { quasi-open, }|\Omega| \leq m, \Phi \in W^{1, \infty}(D),\|\nabla \Phi\|_{L^{\infty}(D)} \leq \tau\right\}
$$

admits a solution $\left(\Omega^{*}, \nabla \Phi^{*}\right)$. Moreover, if $D$ is connected, then any optimal set $\Omega^{*}$ has the following properties:

(1) $\Omega^{*}$ is an open set;

(2) $\Omega^{*}$ has finite perimeter;

(3) $\Omega^{*}$ saturates the constraint, that is, $\left|\Omega^{*}\right|=m$;

The free boundary $\partial \Omega^{*} \cap D$ can be decomposed in the disjoint union of a regular part Reg $\left(\partial \Omega^{*} \cap D\right)$ and a singular part $\operatorname{Sing}\left(\partial \Omega^{*} \cap D\right)$, where:

(4) $\operatorname{Reg}\left(\partial \Omega^{*} \cap D\right)$ is locally the graph of a $C^{1, \alpha}$-regular function for any $\alpha<1$;

(5) for a universal constant $d^{*} \in\{5,6,7\}$ (see Definition [5.39), Sing $\left(\partial \Omega^{*} \cap D\right)$ is:

- empty if $d<d^{*}$;

- discrete if $d=d^{*}$;

- of Hausdorff dimension at most $\left(d-d^{*}\right)$ if $d>d^{*}$.

If the boundary $\partial D$ is $C^{1,1}$, then the boundary $\partial \Omega^{*}$ can be decomposed in the disjoint union of a regular part $\operatorname{Reg}\left(\partial \Omega^{*}\right)$ and a singular part $\operatorname{Sing}\left(\partial \Omega^{*}\right)$, where: 
(6) $\operatorname{Reg}\left(\partial \Omega^{*}\right)$ is an open subset of $\partial \Omega^{*}$ and locally the graph of a $C^{1,1 / 2}$ function; moreover, $\operatorname{Reg}\left(\partial \Omega^{*}\right)$ contains both $\operatorname{Reg}\left(\partial \Omega^{*} \cap D\right)$ and $\partial \Omega^{*} \cap \partial D$;

(7) $\operatorname{Sing}\left(\partial \Omega^{*}\right)=\operatorname{Sing}\left(\partial \Omega^{*} \cap D\right)$.

In fact, our result is more general. Precisely, we prove the regularity of the optimal sets for $\lambda_{1}(\cdot, \nabla \Phi)$ with fixed vector field $\nabla \Phi$ (see Theorem [1.5] and Remark [1.7).

For $m, \tau, \Omega$ and $V$ as in (1.1), Hamel, Nadirashvili and Russ [29], proved the lower bound

$$
\lambda_{1}\left(B, \tau \frac{x}{|x|}\right) \leq \lambda_{1}(\Omega, V),
$$

where $B$ is the ball of Lebesgue measure $m$ centered in zero; moreover, there is an equality in (1.3), if and only if, up to translation, $\Omega=B$ and $V(x)=\tau \frac{x}{|x|}$. In other words, the couple $\left(B, \tau \frac{x}{|x|}\right)$ is (up to translation) the unique solution of the shape optimization problem

$$
\min \left\{\lambda_{1}(\Omega, V): \Omega \subset \mathbb{R}^{d},|\Omega|=m,\|V\|_{L^{\infty}} \leq \tau\right\} .
$$

We notice that a symmetrization technique in the spirit of [29] cannot be applied to the problem (1.1). In fact, the presence of the constraint $D$ makes impossible to determine explicitly the shape of the optimal domains or the precise analytic expression of the optimal vector fields, except in the trivial case when a ball of measure $m$ fits into $D$. Thus, we first establish the existence of an optimal domain $\Omega$ in the larger (relaxed) class of quasi-open sets and we then study the regularity of the optimal shapes through variational free boundary techniques. We stress that, in the case of a generic vector field $V$, the principal eigenvalue $\lambda_{1}(\Omega, V)$ does not have a variational formulation but is only determined trough the solution of a certain PDE on $\Omega$. In particular, the shape cost functional in (1.1) cannot be written in terms of a variational minimization problem involving integral cost functionals on $\Omega$. This makes the extension of the functional $\lambda_{1}(\cdot, V)$ to a $(\gamma-)$ continuous functional on the class of quasi-open sets a non trivial problem.

In the case $\tau=0,(1.1)$ and $(1.2)$ are reduced to the classical shape optimization problem

$$
\min \left\{\lambda_{1}(\Omega): \Omega \subset D,|\Omega| \leq m\right\},
$$

where $\lambda_{1}(\Omega)$ is the first eigenvalue of the Dirichlet Laplacian on $\Omega$. For the problem (1.5), the existence of an optimal (quasi-open) set was proved by Buttazzo and Dal Maso in [13, the fact that the optimal sets are open (Theorem 1.2(1)) was proved by Briançon and Lamboley in [6], the estimate on the perimeter of the optimal set (Theorem [1.2 (2)) is due to Bucur (see [8]), the regularity of the free boundary $\operatorname{Reg}\left(\partial \Omega^{*} \cap D\right)$ was again proved in [6]; the estimate on the dimension of the singular set $\operatorname{Sing}\left(\partial \Omega^{*} \cap D\right)$ was obtained in 34. Even for the classical problem (1.5) the regularity up to the boundary of the box $D$ (Theorem 1.2 (6) and (7)) is new.

Remark 1.3 (On the regularity of the optimal shapes for spectral functionals). The regularity of the optimal shapes for the eigenvalues of the Laplacian was an object of an intense study in the last years. As mentioned above, a regularity result, for the optimal sets for the first eigenvalue of the Laplacian, was proved Briançon and Lamboley in [6]. The regularity of the optimal sets for more general spectral functionals was studied in [10, [34, [?] and [?]. An alternative approach in dimension two, based on the epiperimetric inequality from [38, was recently introduced in 37, where Theorem 1.2 (6) is proved in the case $\tau=0$ and $d=2$. We notice that the method from [37. can be applied to give an alternative proof of Theorem 1.2 (6) in the case $\tau>0$, but the restriction on the dimension is required by the epiperimetric inequality and for now cannot be removed.

Remark 1.4 (On the existence of optimal shapes). The existence of optimal shapes in a bounded open set (box) $D \subset \mathbb{R}^{d}$ is a consequence of the theory of Buttazzo and Dal Maso (see [13] and the books [9] and [30]) for general shape optimization problems of the form

$$
\min \{\mathcal{F}(\Omega): \Omega \subset D \text { quasi-open, }|\Omega| \leq m\},
$$

for shape cost functionals $\mathcal{F}$ with the following properties: 
- $\mathcal{F}$ is decreasing with respect to the set inclusion;

- $\mathcal{F}$ is lower semi-continuous with respect to the $(\gamma$-)convergence of sets.

We notice that in the case when $\mathcal{F}$ is a function of the spectrum of the Dirichlet Laplacian on $\Omega$, the existence of an optimal set can be obtained directly (see for instance [39]). In fact, if $\mathcal{F}(\Omega)=\lambda_{1}(\Omega)$, then given a minimization sequence of quasi-open sets $\Omega_{n}$ for (1.6), and setting $u_{n}$ to be the first eigenfunction of the Dirichlet Laplacian on $\Omega_{n}$, it is not hard to check that, up to a subsequence, $u_{n}$ converges weakly in $H_{0}^{1}(D)$ to a function $u \in H_{0}^{1}(D)$ and that the (quasi-open) set $\Omega:=\{u>0\}$ is a solution to (1.6). This elementary argument works not only for $\lambda_{1}$, but can also be reproduced for general spectral functionals of the form $\mathcal{F}(\Omega)=F\left(\lambda_{1}(\Omega), \ldots, \lambda_{k}(\Omega)\right)$, and also for most of the shape cost functionals present in the literature. We stress that this is not the case of the functional $\mathcal{F}(\Omega)=\lambda_{1}(\Omega, V)$. Even if $\lambda_{1}(\cdot, V)$ is still monotone and $\gamma$-continuous (as we will prove in Section 4), its non-variational nature does not allow to use the elementary argument described above; thus, the only way to obtain the existence of an optimal set is through the Buttazzo-Dal Maso theory.

Optimal shapes for a fixed vector field. In this paper, we also study the case in which only the shape $\Omega$ is variable, while the vector field $V$ is fixed. Precisely, we consider the shape optimization problem

$$
\min \left\{\lambda_{1}(\Omega, V): \Omega \subset D,|\Omega| \leq m\right\},
$$

where both the upper bound $m$ of the Lebesgue measure of the domain $\Omega$ and the vector field $V$ are fixed. In this case the geometry of the optimal sets is affected both by the geometric constraint $\Omega \subset D$ and the vector field $V$. We notice that in this case it is the inclusion constraint that provides the compactness necessary for the existence of an optimal set. We show that the shape functional $\Omega \mapsto \lambda_{1}(\Omega, V)$ is lower semi-continuous with respect to the so-called $\gamma$-convergence of sets and then we obtain the existence of optimal sets by the general result discussed in Remark 1.4. Furthermore, when the vector field is the gradient of a Lipschitz function, we prove a regularity result for the optimal sets. Our main result is the following.

Theorem 1.5 (Existence and regularity of optimal shapes for a fixed vector field). Let $D$ be a bounded open set in $\mathbb{R}^{d}$. Let $m \in(0,|D|)$ and let the vector field $V: D \rightarrow \mathbb{R}^{d}$ be such that $\|V\|_{L^{\infty}}=\tau<+\infty$. Then the shape optimization problem

$$
\min \left\{\lambda_{1}(\Omega, V): \Omega \subset D \text { quasi-open, }|\Omega| \leq m\right\}
$$

admits a solution $\Omega^{*} \subset D$. Moreover, if $D$ is connected and the vector field $V$ is of the form $V=\nabla \Phi$, where $\Phi: D \rightarrow \mathbb{R}$ is a given Lipschitz function, then any solution $\Omega^{*}$ of (1.8) has the following properties:

(1) $\Omega^{*}$ is an open set;

(2) $\Omega^{*}$ has finite perimeter;

(3) $\Omega^{*}$ saturates the constraint, that is, $\left|\Omega^{*}\right|=m$;

The free boundary $\partial \Omega^{*} \cap D$ can be decomposed in the disjoint union of a regular part $\operatorname{Reg}\left(\partial \Omega^{*} \cap D\right)$ and a singular part $\operatorname{Sing}\left(\partial \Omega^{*} \cap D\right)$, where:

(4) $\operatorname{Reg}\left(\partial \Omega^{*} \cap D\right)$ is locally the graph of a $C^{1, \alpha}$-regular function for any $\alpha<1$;

(5) for a universal constant $d^{*} \in\{5,6,7\}$ (see Definition [5.39), Sing $\left(\partial \Omega^{*} \cap D\right)$ is:

- empty if $d<d^{*}$;

- discrete if $d=d^{*}$;

- of Hausdorff dimension at most $\left(d-d^{*}\right)$ if $d>d^{*}$.

If the boundary $\partial D$ is $C^{1,1}$, then the boundary $\partial \Omega^{*}$ can be decomposed in the disjoint union of a regular part $\operatorname{Reg}\left(\partial \Omega^{*}\right)$ and a singular part $\operatorname{Sing}\left(\partial \Omega^{*}\right)$, where:

(6) $\operatorname{Reg}\left(\partial \Omega^{*}\right)$ is an open subset of $\partial \Omega^{*}$ and locally the graph of a $C^{1,1 / 2}$ function; moreover, $\operatorname{Reg}\left(\partial \Omega^{*}\right)$ contains both $\operatorname{Reg}\left(\partial \Omega^{*} \cap D\right)$ and $\partial \Omega^{*} \cap \partial D$;

(7) $\operatorname{Sing}\left(\partial \Omega^{*}\right)=\operatorname{Sing}\left(\partial \Omega^{*} \cap D\right)$. 
Remark 1.6 (On the optimal regularity of the free boundary). The regularity of the boundary of an optimal set $\Omega^{*}$ to the problem (1.8) at contact points of the free boundary with the box cannot exceed $C^{1,1 / 2}$ even if the vector field is smooth. Indeed, Chang-Lara and Savin proved in [18 that the boundary of $\Omega_{u}$, where $u$ is a solution of the free boundary problem (5.40) in $\Omega_{u}=\Omega^{*}$, is at most $C^{1,1 / 2}$ regular.

Remark 1.7 (Regularity of the optimal shapes for variable vector field). We notice that if the couple $(\Omega, V)$ is a solution to the shape optimization problem (1.1) or (1.2), then fixing $V$, we obtain that $\Omega$ is a solution to (1.8). In particular, the regularity part of Theorem 1.2 is a consequence of Theorem 1.5

Outline of the proof and plan of the paper. Throughout the paper the bounded open set $D \subset \mathbb{R}^{d}$ is fixed and is assumed to be (at least $C^{1,1}$ ) smooth.

In the sections 2, 3 and 4, we prove our main existence results (Theorem 1.1) and the existence of an optimal domain for a fixed vector field (Theorem 1.5), as well as the existence of an optimal domain in Theorem 1.2.

In Section 2 we recall several central definitions and results in the $\gamma$-convergence theory of quasi-open sets. In particular, we show that the (classical) $\gamma$-convergence of a sequence of quasiopen sets is equivalent to the strong convergence of the sequence of resolvent operators for $L=$ $-\Delta+V(x) \cdot \nabla$ on each of the sets.

In Section 3, Theorem 3.3 and Corollary [3.9, we prove that the principal eigenvalue is welldefined on every quasi-open set $\Omega \subset D$, that is, there exists a (real) eigenvalue $\lambda_{1}(\Omega, V) \in \mathbb{R}$ of the operator $L=-\Delta+V(x) \cdot \nabla$, such that for any other (complex) eigenvalue $\lambda \in \mathbb{C}$ we have $\lambda_{1}(\Omega, V) \leq \operatorname{Re} \lambda$. In the same section, we establish the continuity of the functional $\Omega \mapsto \lambda_{1}(\Omega, V)$ with respect to the $\gamma$-convergence (Proposition 3.7) and the fact that the principal eigenvalue is decreasing with respet to the set inclusion (Remark 3.2).

In Section 4 we prove our main existence results. The existence of the optimal set for a fixed vector field $V$ (Theorem 1.5) follows by the classical Buttazzo-Dal Maso theorem (Theorem 2.5). We give the precise statement in Theorem 4.1. The proof of Theorem 1.1 requires a more refined argument. The reason is the following: consider a (minimizing) sequence $\left(V_{n}, \Omega_{n}\right)$ of vector fields $V_{n}$ and quasi-open sets $\Omega_{n}$ with eigenfunctions $u_{n} \in H_{0}^{1}\left(\Omega_{n}\right)$ of $L_{n}=-\Delta+V_{n} \cdot \nabla$, solutions of

$$
-\Delta u_{n}+V_{n} \cdot \nabla u_{n}=\lambda_{1}\left(\Omega_{n}, V_{n}\right) u_{n} \quad \text { in } \quad \Omega_{n}, \quad \int_{D} u_{n}^{2} d x=1, \quad u_{n} \in H_{0}^{1}\left(\Omega_{n}\right) .
$$

Let us suppose for simplicity that: $\Omega_{n} \gamma$-converge to a quasi-open set $\Omega ; u_{n}$ converge to a function $u \in H_{0}^{1}(\Omega)$ both strongly $L^{2}(D)$ and weakly $H_{0}^{1}(D) ; V_{n}$ converge weakly (in $L^{2}(D)$ ) to some $V \in L^{\infty}\left(D ; \mathbb{R}^{d}\right)$. Now, the limit function $u$ solves a PDE in $\Omega$, which involves the (weak) limit of the term $V_{n} \cdot \nabla u_{n}$, but a priori this might be different from $V \cdot \nabla u$. In order to solve this issue, in Section 4, we first prove that, on any fixed quasi-open set $\Omega$, there exists an optimal vector field (see Theorem 4.2). We then replace the vector fields $V_{n}$ of the minimizing sequence $\left(V_{n}, \Omega_{n}\right)$ by the optimal vector field $V_{n}^{*}$ on each domain. Finally, we use the precise expression of $V_{n}^{*}$ to prove that the limit function $u$ is an eigenfunction of $-\Delta+V \cdot \nabla$ on $\Omega$ and we obtain that $\lambda_{1}\left(\Omega_{n}, V_{n}^{*}\right)$ converges to $\lambda_{1}\left(\Omega, V^{*}\right)$, which concludes the proof (see Theorem 4.3). We cannot apply the same argument for Theorem 1.2, since the optimal vector field might not be a gradient. On the other hand, for gradient vector fields the first eigenvalue is a variational functional, namely

$$
\lambda_{1}(\Omega, \nabla \Phi)=\min _{u \in H_{0}^{1}(\Omega) \backslash\{0\}} \frac{\int_{D} e^{-\Phi}|\nabla u|^{2} d x}{\int_{D} e^{-\Phi} u^{2} d x},
$$

and the existence of an optimal set can be obtained directly (see Theorem 4.5).

In Section 5, for a fixed drift $V=\nabla \Phi$, we prove the regularity of the optimal sets for $\lambda_{1}(\cdot, \nabla \Phi)$ (Theorem[1.5). In particular, this implies the regularity of the optimal sets in the case when both the set $\Omega$ and the vector field $\nabla \Phi$ may vary (see Theorem 1.2). Our argument relies in an essential way on the variational formulation of $\lambda_{1}(\Omega, V)$. More precisely, we show (see Lemma 5.1) that, 
if $V=\nabla \Phi$ is fixed and $\Omega \subset D$ is a solution of (1.8), then the corresponding eigenfunction solves the free boundary problem

$$
\min \left\{\int_{D} e^{-\Phi}|\nabla u|^{2} d x: u \in H_{0}^{1}(D), u \geq 0,|\{u \neq 0\}| \leq m, \int_{D} e^{-\Phi} u^{2} d x=1\right\} .
$$

This is a one-phase free boundary problem, similar to the one studied in the seminal paper of Alt and Caffarelli [2] on the local minimizers of the one-phase functional

$$
u \mapsto \int|\nabla u|^{2} d x+|\{u>0\}| .
$$

Nevertheless, there are four differences with repect to the classical one-phase problem [2].

(i) the presence of the variable coefficient $e^{-\Phi}$ in the functional;

(ii) the presence of the integral constraint $\int e^{-\Phi} u^{2} d x=1$;

(iii) the presence of the measure constraint $|\{u>0\}| \leq m$;

(iv) the presence of the inclusion constraint $\{u>0\} \subset D$ (equivalent to $u \in H_{0}^{1}(D)$ ).

The variable coefficient $e^{-\Phi}$ introduces several technical difficulties, but does not have an influence on the overall strategy. The issues with the integral constraint are of similar nature. In fact, we are able to deal with this term (see Subsection 5.3 and Remark 5.9) by reformulating the free boundary problem (1.9) in terms of the functional

$$
J(v):=\int_{D}|\nabla v|^{2} e^{-\Phi} d x-\lambda_{m} \int_{D} v^{2} e^{-\Phi} d x,
$$

where $\lambda_{m}$ is the value of the minimum in (1.9). In fact, one easily checks that, if $u$ is a solution of (1.9), then $u$ is also a solution to the free boundary problem

$$
J(u) \leq J(v) \quad \text { for every } \quad v \in H_{0}^{1}(D) \quad \text { such that } \quad\left|\Omega_{v}\right| \leq m,
$$

where, for any function $v$, we set $\Omega_{v}:=\{v>0\}=\{x \in \Omega: v(x)>0\}$.

The measure constraint in free boundary problems first appeared in the work of Aguilera, Alt and Caffarelli [1. In fact, it is not hard to check that, at least formally, the solution $u$ should satisfy the optimality condition

$$
|\nabla u|=\sqrt{\Lambda_{u} e^{\Phi}} \quad \text { on the free boundary } \partial \Omega_{u} \cap D,
$$

where $\Lambda_{u}$ is a Lagrange multiplier formally arising in the minimization of the functional $J(u)$ under the constraint $\left|\Omega_{u}\right|=m$ (see Subsection [5.4). Thus, at least formally, there is no difference between the classical one-phase free boundary problem and the problem with a measure constraint. In practice, dealing with the measure constraint is an hardeous task. In fact, the Lagrange multiplier $\Lambda_{u}$ arises by applying internal variation to the function $u$, which by itself cannot be used to deduce even the basic qualitative properties of the solution $u$ as, for instance, the Lipschitz continuity and the non-degeneracy (in other words, at the moment, the regularity of the stationary free boundaries is not known). Our approach is different from the one in [1] and is inspired by the works of Briançon-Lamboley [6] and Briançon [7]. In fact, we aim to tranform the problem (1.10) into

$$
J(u)+\Lambda_{u}\left|\Omega_{u}\right| \leq J(v)+\Lambda_{u}\left|\Omega_{v}\right| \quad \text { for every } \quad v \in H_{0}^{1}(D) .
$$

Now, it is not possible to re-write (1.10) precisely in this form. Instead, we prove that

$$
J(u)-J(v) \leq\left\{\begin{array}{lllll}
\left(\Lambda_{u}+\varepsilon\right)\left(\left|\Omega_{v}\right|-\left|\Omega_{u}\right|\right) & \text { for every } & v \in H_{0}^{1}(D) & \text { such that } & \left|\Omega_{v}\right| \geq m ; \\
\left(\Lambda_{u}-\varepsilon\right)\left(\left|\Omega_{v}\right|-\left|\Omega_{u}\right|\right) & \text { for every } & v \in H_{0}^{1}(D) & \text { such that } & \left|\Omega_{v}\right| \leq m ;
\end{array}\right.
$$

where the constant $\varepsilon$ improves at small scales, that is, if we consider competitors $v$ that differ from $u$ only in a small ball of radius $r$, then $\varepsilon$ can be chosen in a function of $r, \varepsilon=\varepsilon(r)$, which is such that $\varepsilon(r) \rightarrow 0$ as $r \rightarrow 0$. In this part of the proof (Subsection 5.5) we follow the analysis of [6], except in one fundamental point. In fact, the approach of Briançon and Lamboley requires that 
the Lagrange multiplier $\Lambda_{u}$ is not vanishing, which is not a priori known (see Proposition 5.12); in [6] the issue is solved by the method in [7]. In this paper, we give a different argument to prove that the Lagrange multiplier is non trivial. Our approach is based on the Almgren monotonicity formula, and the fact that it implies the non-degeneracy of the solution $u$. We give the proof of the appendix, since the argument is very general (based only on the stationarity condition) and might be of independent interest. We also notice that this simplifies the proof of (1.12) and reduces it to three fundamental steps (see Theorem 5.16).

Our proof of Theorem 1.5 is general and can be applied to the classical one-phase problem [2], to the one-phase problem with measure constraint [1] and to shape optimization problems as for instance the one of [6]. Our approach is different from (and alternative to) the one of [2], [1] and [6], as we do not use the regularity result of Alt and Caffarelli [2]. In fact, in order to prove the regularity of the flat free boundaries (Subsection 5.9), we prove that the optimality condition on the free boundary holds in viscosity sense (see Lemma 5.30) and then we apply the general results of De Silva [21] (for the regularity of the free boundary $\partial \Omega_{u} \cap D$ ) and the recent result of Chang-Lara and Savin [18] (for the regularity at the contact points $\partial \Omega_{u} \cap \partial D$ ). Finally, the estimate on the dimension of the singular set (Subsection 5.10) is a consequence of the Weiss' (quasi-)monotonicity formula (Lemma 5.37).

\section{Preliminaries}

In this section we recall the main definitions and the properties of the quasi-open sets, the $\gamma$-convergence and the weak- $\gamma$-convergence.

\subsection{Capacity, quasi-open sets and quasi-continuous functions.}

The capacity of a set $E \subset \mathbb{R}^{d}$ is defined as

$$
\operatorname{cap}(E):=\inf \left\{\|u\|_{H^{1}}^{2}: u \in H^{1}\left(\mathbb{R}^{d}\right), u \geq 1 \text { in a neighborhood of } E\right\},
$$

where $H^{1}\left(\mathbb{R}^{d}\right)$ is the Sobolev space equipped with the norm $\|u\|_{H^{1}}^{2}=\int_{\mathbb{R}^{d}}\left(|\nabla u|^{2}+u^{2}\right) d x$.

We say that a property holds quasi-everywhere (q.e.) if it holds on the complementary of a set of zero capacity.

A set $\Omega \subset \mathbb{R}^{d}$ is said to be quasi-open if there exists a decreasing sequence $\left(\omega_{n}\right)_{n \geq 1}$ of open sets such that, for every $n \geq 1, \Omega \cup \omega_{n}$ is an open set and $\lim _{n \rightarrow \infty} \operatorname{cap}\left(\omega_{n}\right)=0$.

A function $u: \mathbb{R}^{d} \rightarrow \mathbb{R}$ is said to be quasi-continuous if there exists a decreasing sequence $\left(\omega_{n}\right)_{n \geq 1}$ of open sets such that $\lim _{n \rightarrow \infty} \operatorname{cap}\left(\omega_{n}\right)=0$ and the restriction of $u$ to $\mathbb{R}^{d} \backslash \omega_{n}$ is continuous.

It is well-known (see for instance [25, Theorem 1, Section 4.8]) that, for every $u \in H^{1}\left(\mathbb{R}^{d}\right)$, there exists a quasi-continuous representative $\tilde{u}$ of $u$, which is unique up to a set of zero capacity. From now on we will identify a function $u \in H^{1}\left(\mathbb{R}^{d}\right)$ with its quasi-continuous representative. We note that, by definition of a quasi-open set and a quasi-continuous function, for every $u \in H^{1}\left(\mathbb{R}^{d}\right)$, the set $\Omega_{u}:=\{u>0\}=\left\{x \in \mathbb{R}^{d} \mid u(x)>0\right\}$ is a quasi-open set ([30, Proposition 3.3.41]). On the other hand, for every quasi-open set $\Omega$, there exists a function $u \in H^{1}\left(\mathbb{R}^{d}\right)$ such that $\Omega=\Omega_{u}$ up to a set of zero capacity that is, the quasi-open sets are superlevel sets of Sobolev functions.

For any set $E \subset \mathbb{R}^{d}$, the Sobolev space $H_{0}^{1}(E) \subset H^{1}\left(\mathbb{R}^{d}\right)$ is defined as

$$
H_{0}^{1}(E):=\left\{u \in H^{1}\left(\mathbb{R}^{d}\right): u=0 \text { q.e. in } \mathbb{R}^{d} \backslash E\right\} .
$$

Note that, whenever $E$ is open, this definition coincides with the usual definition of $H_{0}^{1}(E)$ as the closure of $C_{c}^{\infty}(E)$ with respect to the norm $\|\cdot\|_{H^{1}}, C_{c}^{\infty}(E)$ being the set of smooth functions compactly supported in $E$ (see for instance [30, Theorem 3.3.42]). For any set $E \subset \mathbb{R}^{d}$ there is a quasi-open set $\tilde{E} \subset \mathbb{R}^{d}$ such that $\operatorname{cap}(\tilde{E} \backslash E)=0$ and $H_{0}^{1}(\tilde{E})=H_{0}^{1}(E)$. Roughly speaking, the quasi-open sets are the natural domains for the Sobolev space $H_{0}^{1}$. We notice that, for every quasi-open set $E, H_{0}^{1}(E)$ is a closed subspace of $H^{1}\left(\mathbb{R}^{d}\right)$; if $E_{1} \subset E_{2}$ are two quasi-open sets, then $H_{0}^{1}\left(E_{1}\right) \subset H_{0}^{1}\left(E_{2}\right)$ and the two sets $E_{1}$ and $E_{2}$ coincide q.e. if and only if $H_{0}^{1}\left(E_{1}\right)=H_{0}^{1}\left(E_{2}\right)$. 
2.2. PDEs on quasi-open sets. Let $D \subset \mathbb{R}^{d}$ be a given open set and $\Omega \subset D$ be a quasi-open set of finite Lebesgue measure. For every quasi-open set $\Omega \subset D$ and every function $f \in L^{2}(\Omega)$, the Lax-Milgram theorem and the Poincaré inequality ensure that there is a unique solution $u \in H_{0}^{1}(\Omega)$ of the problem

$$
-\Delta u=f \quad \text { in } \quad \Omega, \quad u \in H_{0}^{1}(\Omega),
$$

where the PDE is intended in the weak sense

$$
\int_{\Omega} \nabla u \cdot \nabla \varphi d x=\int_{\Omega} f \varphi d x, \text { for every } \varphi \in H_{0}^{1}(\Omega) .
$$

In particular, taking $u=\varphi$, we notice that $\|\nabla u\|_{L^{2}(\Omega)} \leq\|f\|_{L^{2}(\Omega)}\|u\|_{L^{2}(\Omega)}$. Now since $\Omega$ has a finite Lebesgue measure, there is a constant $C_{\Omega}$ such that $\|u\|_{H^{1}} \leq C_{\Omega}\|\nabla u\|_{L^{2}}$ for every $u \in$ $H_{0}^{1}(\Omega)$. Thus, we get that $\|u\|_{H^{1}} \leq C_{\Omega}\|f\|_{L^{2}}$.

The resolvent operator $R_{\Omega}^{-\Delta}: L^{2}(D) \rightarrow L^{2}(D)$ is defined as $R_{\Omega}^{-\Delta}(f):=u$ and is a linear, continuous, self-adjoint, positive operator such that $R_{\Omega}^{-\Delta}\left(L^{2}(D)\right) \subset H_{0}^{1}(\Omega)$. Moreover, thanks to the compact embedding $H_{0}^{1}(\Omega) \hookrightarrow L^{2}(\Omega)$, the resolvent $R_{\Omega}^{-\Delta}$ is also compact.

The usual comparison and weak maximum principles hold in this setting. Precisely, we have:

- if $f \in L^{2}(D)$ is a positive function and $\Omega_{1} \subset \Omega_{2} \subset D$ are two quasi-open sets, then $w_{\Omega_{1}} \leq w_{\Omega_{2}}$.

- if $\Omega$ is a quasi-open set and $f, g \in L^{2}(\Omega)$ are such that $f \leq g$ in $\Omega$, then $R_{\Omega}^{-\Delta}(f) \leq R_{\Omega}^{-\Delta}(g)$.

In the sequel we denote by $w_{\Omega}$ (and sometimes also by $R_{\Omega}^{-\Delta}(1)$ ) the solution of

$$
-\Delta w_{\Omega}=1 \quad \text { in } \Omega, \quad w_{\Omega} \in H_{0}^{1}(\Omega) .
$$

This function is sometimes called torsion or energy function and is useful, in particular, to define the topology of the $\gamma$-convergence on the family of quasi-open sets, which is the purpose of the next section. In the following proposition we summarize the main properties of the function $w_{\Omega}$ (see for instance [39, Proposition 3.50, Remark 3.53, Lemma 3.125, Proposition 3.72]).

Proposition 2.1 (Properties of the torsion function $w_{\Omega}$ ).

(1) There is a dimensional constant $C_{d}>0$ such that

$$
\left\|\nabla w_{\Omega}\right\|_{L^{2}} \leq C_{d}|\Omega|^{\frac{d+2}{2 d}} \quad \text { and } \quad\left\|w_{\Omega}\right\|_{L^{\infty}} \leq C_{d}|\Omega|^{2 / d} .
$$

(2) Let $\Omega_{1}, \Omega_{2} \subset D$ be two quasi-open sets. Then we have the estimate

$$
\int_{D}\left(w_{\Omega_{1}}-w_{\Omega_{1} \backslash \Omega_{2}}\right) d x \leq \operatorname{cap}\left(\Omega_{2}\right)\left\|w_{\Omega_{1}}\right\|_{L^{\infty}\left(\Omega_{1}\right)}^{2} .
$$

(3) $H_{0}^{1}(\Omega)=H_{0}^{1}\left(\left\{w_{\Omega}>0\right\}\right)$. In particular, $\Omega=\left\{w_{\Omega}>0\right\}$ up to a set of zero capacity.

In the sequel we make the convention to extend to $D$ any vector field $V \in L^{\infty}\left(\Omega, \mathbb{R}^{d}\right)$ and any function $u \in H_{0}^{1}(\Omega)$ by letting it equal to 0 on $D \backslash \Omega$ so that $V \in L^{\infty}\left(D, \mathbb{R}^{d}\right)$ and $u \in H_{0}^{1}(D)$.

We notice that, given a drift $V \in L^{\infty}\left(\Omega, \mathbb{R}^{d}\right)$, the bilinear form associated to the operator $L=-\Delta+V \cdot \nabla$ may not be coercive on $H_{0}^{1}(\Omega)$. Thus, in order to define the resolvent of $L=-\Delta+V \cdot \nabla$, we consider a large enough constant $c>0$ (depending only on $\|V\|_{L^{\infty}(\Omega)}$ ), for which there exists a positive constant $\delta>0$ such that

$$
\delta \int_{D}\left(|\nabla u|^{2}+u^{2}\right) d x \leq \int_{D}\left(|\nabla u|^{2}+(V \cdot \nabla u) u+c u^{2}\right) d x, \quad \text { for every } \quad u \in H_{0}^{1}(\Omega) .
$$

The bilinear form associated to the operator $L^{\prime}=L+c$ is hence coercive on $H_{0}^{1}(\Omega)$. Note that

$$
\text { if }\|V\|_{L^{\infty}} \leq \tau, \quad \text { then we can take any } \quad 0<\delta<1 \quad \text { and } \quad c \geq \delta+\frac{\tau^{2}}{4(1-\delta)} .
$$

Therefore, thanks to Lax Milgram theorem, we define the resolvent $R_{\Omega}^{L^{\prime}}: L^{2}(D) \rightarrow L^{2}(D)$ as the compact (non self-adjoint) operator, which maps $f \in L^{2}(\Omega)$ to the unique solution of the problem

$$
L^{\prime} u=f \quad \text { in } \Omega, \quad u \in H_{0}^{1}(\Omega),
$$


which is intended in the weak sense

$$
\int_{\Omega}(\nabla u \cdot \nabla \varphi+(V \cdot \nabla u) \varphi+c u \varphi) d x=\int_{\Omega} f \varphi d x, \quad \text { for every } \varphi \in H_{0}^{1}(\Omega) .
$$

2.3. The $\gamma$-convergence and the weak- $\gamma$-convergence. In this subsection we briefly recall the definition and the main properties of the $\gamma$-convergence of (quasi-open) sets.

Definition 2.2 ( $\gamma$-convergence and weak- $\gamma$-convergence). Let $D \subset \mathbb{R}^{d}$ be a given open set of finite Lebesgue measure, $\left(\Omega_{n}\right)_{n \geq 1}$ be a sequence of quasi-open sets and let $\Omega$ be a quasi-open set, all included in $D$. We say that

- $\Omega_{n} \gamma$-converges to $\Omega$, if $w_{\Omega_{n}}$ converges to $w_{\Omega}$ strongly in $L^{2}(D)$;

- $\Omega_{n}$ weak- $\gamma$-converges to $\Omega$, if there exists $w \in H_{0}^{1}(D)$ such that $\Omega=\{w>0\}$ and $w_{\Omega_{n}}$ converges to $w$ in $L^{2}(D)$.

Though the $\gamma$-convergence is not compact on the family of quasi-open sets (see for instance 19 and [30, $\S 3.2 .6]$ for an example), it is easy to see that the weak- $\gamma$-convergence is: by (2.1), up to a subsequence, $w_{\Omega_{n}}$ weakly converges in $H_{0}^{1}(D)$ to some $w \in H_{0}^{1}(D)$ and hence $\Omega_{n}$ weak- $\gamma$-converges to the quasi-open set $\Omega:=\{w>0\}$. To deal with the non-compactness of the $\gamma$-convergence we will use the following Lemma (see for example [12] and [30, Lemma 4.7.11]).

Lemma 2.3. Let $\left(\Omega_{n}\right)_{n>1} \subset D$ be a sequence of quasi-open sets that weak- $\gamma$-converges to the quasi-open set $\Omega \subset D$. Then there exists a subsequence of $\left(\Omega_{n}\right)_{n>1}$, still denoted by $\left(\Omega_{n}\right)_{n>1}$, and a sequence $\left(\tilde{\Omega}_{n}\right)_{n \geq 1} \subset D$ of quasi-open sets satisfying $\Omega_{n} \subset \tilde{\Omega}_{n}$, such that $\tilde{\Omega}_{n} \gamma$-converges to $\Omega$.

The following lemma is a direct consequence of the definition of the weak- $\gamma$-convergence and the fact that for every quasi-open set $\Omega=\left\{w_{\Omega}>0\right\}$ (the detailed proof can be found for example in [12] and [39, Lemma 2.2.21]).

Lemma 2.4 (Lower semi-continuity of the Lebesgue measure). Let $\left(\Omega_{n}\right)_{n \geq 1}$ be a sequence of quasi-open sets in $D$ weak- $\gamma$-converging to $\Omega \subset D$, then $|\Omega| \leq \liminf _{n \rightarrow+\infty}\left|\Omega_{n}\right|$.

As was shown in [9] and [12, the following theorem, first proved in [13, is an immediate consequence of Lemma 2.3 and Lemma 2.4.

Theorem 2.5 (Buttazzo-Dal Maso [13]). Let $\mathcal{F}$ be a functional on the quasi-open sets, which is:

- decreasing with respect to the inclusion of sets;

- lower semi-continuous with respect to the $\gamma$-convergence.

Then, for every bounded open set $D \subset \mathbb{R}^{d}$ and every $0<m \leq|D|$, the shape optimization problem

$$
\min \{\mathcal{F}(\Omega): \Omega \text { quasi-open, } \Omega \subset D,|\Omega| \leq m\}
$$

has a solution.

We will not be able to apply directly Theorem 2.5 to establish the existence of optimal sets for both the problems (1.7) and (1.1) in the class of quasi-open sets. Instead, in Section 4, we will use an argument based only on Lemma 2.3 and Lemma 2.4, but before that we will need to extend the definition of $\lambda_{1}(\Omega, V)$ to the class of quasi-open sets. We do this in Section 3 , where we will use several times the following approximation result.

Lemma 2.6 (Approximation with open and smooth sets). Let $\Omega \subset D$ be a quasi-open set. Then: (1) there is a sequence of open sets $\left(\Omega_{n}\right)_{n \geq 1}$ that $\gamma$-converges to $\Omega$ and is such that $\Omega \subset \Omega_{n} \subset D$ and $\lim _{n \rightarrow+\infty}\left|\Omega_{n}\right|=|\Omega|$;

(2) there is a sequence $\left(\Omega_{n}\right)_{n \geq 1}$ of smooth $\left(C^{\infty}\right)$ open sets contained in $D$, that $\gamma$-converges to $\Omega$.

Proof. The result is well-known; here we give the proof for the readers' convenience.

(1) Let $\left(\omega_{n}\right)_{n>1}$ be a sequence of open sets such that $\lim _{n \rightarrow \infty} \operatorname{cap}\left(\omega_{n}\right)=0$ and $\Omega_{n}=\left(\Omega \cup \omega_{n}\right) \cap D$ is an open set. Then, (2.2) applied to the sets $\Omega_{n}$ and $\omega_{n} \backslash \Omega$ together with the second estimate in (2.1) show that $w_{\Omega_{n}}$ converges to $w_{\Omega}$ in $L^{1}(D)$. Moreover, up to a subsequence, $w_{\Omega_{n}}$ weakly 
converges in $H^{1}(D)$ thanks to the first estimate in (2.1). Since the embedding $H_{0}^{1}(D) \hookrightarrow L^{2}(D)$ is compact, there is a subsequence which converges strongly in $L^{2}(D)$. By uniqueness of the limit in $L^{1}(D)$, it has to be $w_{\Omega}$. Thus, $w_{\Omega_{n}}$ converges in $L^{2}(D)$ to $w_{\Omega}$ and so, $\Omega_{n} \gamma$-converges to $\Omega$. Observe also that one has $\lim _{n \rightarrow+\infty}\left|\Omega_{n}\right|=|\Omega|$ since $\lim _{n \rightarrow+\infty}\left|\omega_{n}\right|=0$.

(2) Firstly, assume that $\Omega$ is an open set. Let $\left(\Omega_{n}\right)_{n>1}$ be an increasing sequence of smooth open sets included in $\Omega$ which Hausdorff converges to $\Omega$. Then, up to a subsequence, $w_{n}:=w_{\Omega_{n}}$ weakly converges in $H_{0}^{1}(D)$ to some $w \in H_{0}^{1}(D)$. But $\Omega_{n}, \Omega$ are open sets such that $\Omega_{n} \subset \Omega$, and since the convergence of $\Omega_{n}$ to $\Omega$ is Hausdorff, we can pass to the limit in the equation

$$
-\Delta w_{n}=1 \quad \text { in } \quad \Omega_{n}
$$

to see that $w$ satisfies

$$
-\Delta w=1 \text { in } \Omega .
$$

This also shows that the sequence of norms $\left\|w_{n}\right\|_{H^{1}(D)}$ converges to $\|w\|_{H^{1}(D)}$, so that the convergence of $w_{n}$ to $w$ is strong in $H^{1}(D)$. Finally, since $\Omega_{n} \subset \Omega$, we get that $w \in H_{0}^{1}(\Omega)$ and hence that $w=w_{\Omega}$. Therefore, the sequence of smooth open sets $\Omega_{n} \gamma$-converges to $\Omega$.

If now $\Omega$ is merely a quasi-open set, we can approximate $\Omega$ by a sequence of open sets which $\gamma$-converges to $\Omega$ thanks to (1). Hence, by approximating these open sets by open smooth sets as above, we get a sequence of smooth open sets which $\gamma$-converges to $\Omega$. Recall that the topology of the $\gamma$-convergence is metrizable (see for example [9]).

Remark 2.7 (The quasi-open sets cannot be $\gamma$-approximated with bigger smooth open sets). In general, we cannot approximate a quasi-open set (or even an open set) $\Omega \subset D$ by a sequence of smooth (say of class $C^{1}$ ) open sets $\left(\Omega_{n}\right)_{n \geq 1}$ which $\gamma$-converges to $\Omega$ and such that $\Omega_{n} \supset \Omega$. Indeed, let $\left(\xi_{n}\right)_{n \geq 1}$ be a dense sequence in $D=(0,1)^{2} \subset \mathbb{R}^{2}$ and pick a sequence $\left(r_{n}\right)_{n \geq 1}$ of positive numbers such that $\sum_{n \geq 1} \pi r_{n}^{2}<1$. Set $\Omega:=\cup_{n \geq 1} B_{r_{n}}\left(\xi_{n}\right) \subset D$. We now claim that if $\Omega_{n} \supset \Omega$ is a smooth open set, then necessarily $\Omega_{n} \supset D$. To see this, let $x_{0} \in D \subset \bar{\Omega} \subset \bar{\Omega}_{n}$. Then if $x_{0} \in \partial \Omega_{n}$, there exist $r>0$ and a smooth, say of class $C^{1}$, function $f: \mathbb{R}^{d} \rightarrow \mathbb{R}$ such that, up to reorienting the axis, we have $\Omega_{n} \cap B_{r}\left(x_{0}\right)=\left\{x \in B_{r}\left(x_{0}\right): x_{d}>f\left(x_{1}, \cdots, x_{d-1}\right)\right\}$. It follows that $B_{r}\left(x_{0}\right) \backslash \bar{\Omega}_{n} \subset D$ is a nonempty open set which does not intersect $\Omega_{n}$. This is in contradiction with $\Omega_{n} \supset \Omega$ since $\Omega$ is a dense open set in $D$. Hence $x \in \Omega_{n}$ and this shows that $D \subset \Omega_{n}$. Now, suppose that $\left(\Omega_{n}\right)_{n>1}$ is a sequence of smooth sets such that $D \supset \Omega_{n} \supset \Omega$. Then $\Omega_{n}=D$ for every $n \geq 1$. Furthermore, the weak maximum principle implies $w_{\Omega}<w_{D}=w_{\Omega_{n}}$ in $D$, where the first inequality is strict since $|\Omega|<|D|=1$. Therefore, $w_{\Omega_{n}}$ cannot strongly converge to $w_{\Omega}$ in $L^{2}(D)$.

We now give a characterization of the $\gamma$-convergence in terms of convergence of resolvent operators. The following theorem is a generalization of [30, Lemma 4.7.3] for the operator $L$.

Theorem 2.8 ( $\gamma$-convergence and operator convergence). Let $D \subset \mathbb{R}^{d}$ be a bounded open set, $\left(\Omega_{n}\right)_{n \geq 1} \subset D$ be a sequence of quasi-open sets and $\Omega \subset D$ be a quasi-open set. The following assertions are equivalent :

(1) the sequence $\left(\Omega_{n}\right)_{n>1} \gamma$-converges to $\Omega$;

(2) for every sequence $\left(f_{n}\right)_{n>1} \in L^{2}(D)$ weakly converging in $L^{2}(D)$ to $f \in L^{2}(D)$, the sequence $\left(R_{\Omega_{n}}^{L}\left(f_{n}\right)\right)_{n \geq 1}$ converges to $R_{\Omega}^{L}(f)$ strongly in $L^{2}(D)$;

(3) the sequence of operators $\left(R_{\Omega_{n}}^{L}\right)_{n \geq 1} \in \mathcal{L}\left(L^{2}(D)\right)$ converges to $R_{\Omega}^{L}$ in the operator norm $\|$. $\|_{\mathcal{L}\left(L^{2}(D)\right)} \cdot$

Proof. It is plain to see that the equivalence between (2) and (3) holds for all sequence of compact operators defined on Hilbert spaces. It then remains to prove that (1) and (2) are equivalent.

$(1) \Rightarrow(2)$. Let $f_{n} \in L^{2}(D)$ be a sequence $L^{2}(D)$-weakly converging to $f \in L^{2}(D)$. Then $\left\|f_{n}\right\|_{L^{2}}$ is uniformly bounded. Moreover, writing $u_{n}=R_{\Omega_{n}}^{L}\left(f_{n}\right)$ we have

$$
\int_{D} f_{n} u_{n} d x=\int_{D}\left(\left|\nabla u_{n}\right|^{2}+\left(V \cdot \nabla u_{n}\right) u_{n}+c u_{n}^{2}\right) d x
$$


Thanks to (2.3) this gives

$$
\frac{1}{2} \int_{D}\left(f_{n}^{2}+u_{n}^{2}\right) d x \geq \delta \int_{D}\left(\left|\nabla u_{n}\right|^{2}+u_{n}^{2}\right) d x,
$$

and therefore

$$
\int_{D} f_{n}^{2} d x \geq(2 \delta-1) \int_{D}\left(\left|\nabla u_{n}\right|^{2}+u_{n}^{2}\right) d x
$$

Taking $\delta \in(1 / 2,1)$, this shows that the sequence $\left\|u_{n}\right\|_{H^{1}(D)}$ is bounded.

Assume now that the conclusion of (2) does not hold. Then there exists $\varepsilon>0$ such that, up to a subsequence, $\left\|R_{\Omega_{n}}^{L}\left(f_{n}\right)-R_{\Omega}^{L}(f)\right\|_{L^{2}(D)} \geq \varepsilon$. Moreover, up to a subsequence, $u_{n}$ weakly converges in $H^{1}(D)$ to some $u \in H_{0}^{1}(D)$, and therefore $g_{n}=f_{n}-V \cdot \nabla u_{n}-c u_{n}$ weakly converges in $L^{2}(D)$ to $g=f-V \cdot \nabla u-c u$. Theorem 2.8 being true for the Laplacian (see [14, Proposition 3.4]), we conclude that $R_{\Omega_{n}}^{-\Delta}\left(g_{n}\right)$ strongly converges in $L^{2}(D)$ to $R_{\Omega}^{-\Delta}(g)$. Thus $R_{\Omega_{n}}^{L}\left(f_{n}\right)=R_{\Omega_{n}}\left(g_{n}\right)$ and $R_{\Omega}^{L}(f)=R_{\Omega}(g)$ imply that $R_{\Omega_{n}}^{L}\left(f_{n}\right)$ strongly converges in $L^{2}(D)$ to $R_{\Omega}^{L}(f)$, which yields a contradiction and therefore proves (2).

(2) $\Rightarrow(1)$. Let $\left(f_{n}\right)_{n>1} \in L^{2}(D)$ be a sequence weakly converging in $L^{2}(D)$ to $f \in L^{2}(D)$. Set $w_{n}:=R_{\Omega_{n}}^{-\Delta}\left(f_{n}\right)$ and $w:=R_{\Omega}^{-\Delta}(f)$. We claim that $w_{n} \rightarrow w$ strongly in $L^{2}(D)$, which, according to [14] and [30, Lemma 4.7.3], implies that $\Omega_{n} \gamma$-converges to $\Omega$. Assume by contradiction that it is not the case, and pick up $\varepsilon>0$ and an increasing function $\varphi: \mathbb{N}^{*} \rightarrow \mathbb{N}^{*}$ such that

$$
\left\|w_{\varphi(n)}-w\right\|_{L^{2}(D)} \geq \varepsilon \quad \text { for every } \quad n \geq 1 \text {. }
$$

Since the sequence $\left(w_{n}\right)_{n \geq 1}$ is bounded in $H_{0}^{1}(D)$, up to a subsequence, there exists a function $z \in H_{0}^{1}(D)$ such that $w_{\varphi(n)}$ converges to $z$ weakly in $H_{0}^{1}(D)$ and strongly in $L^{2}(D)$. Now, since

$$
L w_{n}=f_{n}+V \cdot \nabla w_{n}+c w_{n}:=g_{n} \quad \text { in } \quad \Omega_{n},
$$

and $w_{n} \in H_{0}^{1}\left(\Omega_{n}\right), w_{n}=R_{\Omega_{n}}^{L}\left(g_{n}\right)$. But $g_{\varphi(n)} \rightarrow g:=f+V \cdot \nabla z+c z$ weakly in $L^{2}(D)$, so that, by assumption $(2), w_{\varphi(n)} \rightarrow R_{\Omega}^{L}(g)$ strongly in $L^{2}(D)$. Then the convergence of $w_{\varphi(n)}$ to $z$ yields that $z=R_{\Omega}^{L}(g)$, is a solution of

$$
L z=f+V \cdot \nabla z+c z \quad \text { in } \quad \Omega, \quad z \in H_{0}^{1}(\Omega),
$$

or, in other words, $z=R_{\Omega}^{-\Delta}(f)=w$. Thus, (2.4) provides a contradiction, therefore showing that $w_{n} \rightarrow w$ strongly in $L^{2}(D)$, which means that (1) holds.

\section{The PRInCipal Eigenvalue on Quasi-OPEN SETS}

For a bounded domain $\Omega \subset \mathbb{R}^{d}$ and $V \in L^{\infty}\left(\Omega, \mathbb{R}^{d}\right)$, the principal eigenvalue $\lambda_{1}(\Omega, V)$, of the (non self-adjoint) elliptic operator $L=-\Delta+V \cdot \nabla$ on $\Omega$ with Dirichlet boundary condition on $\partial \Omega$, was defined in [4] by

$$
\lambda_{1}(\Omega, V)=\sup \left\{\lambda \in \mathbb{R}: \exists \phi \in W^{2, d}(\Omega) \quad \text { such that } \phi>0 \quad \text { and } \quad-L \phi+\lambda \phi \leq 0 \text { in } \Omega\right\},
$$

where it was proved that $\lambda_{1}(\Omega, V) \in \mathbb{R}$ has the following properties:

(i) There is a positive eigenfunction $u: \Omega \rightarrow \mathbb{R}$ such that $u \in W_{l o c}^{2, p}(\Omega)$, for all $p \in[1,+\infty)$, and

$$
L u=\lambda_{1}(\Omega, V) u \quad \text { in } \quad \Omega, \quad u \in H_{0}^{1}(\Omega), \quad \int_{\Omega} u^{2} d x=1,
$$

(see [4, Theorem 2.1]).

(ii) $\lambda_{1}(\Omega, V)<\operatorname{Re}(\lambda)$ for every eigenvalue $\lambda \neq \lambda_{1}(\Omega, V)$ of $L$ in $\Omega$ (see [4, Theorem 2.3]).

(iii) The functional $\Omega \mapsto \lambda_{1}(\Omega, V)$ is decreasing with respect to the domain inclusion.

In the sequel we extend the definition of $\lambda_{1}(\Omega, V)$ to quasi-open sets. We first recall that the definition can be extended to an arbitrary open set $\Omega \subset D$ by

$$
\lambda_{1}(\Omega, V)=\inf \lambda_{1}\left(\Omega_{n}, V\right),
$$


where the infimum is taken over all the connected component $\Omega_{n}$ of $\Omega$. Now, in view of property (iii) above, for any quasi-open set $\Omega \subset D$, we define

$$
\lambda_{1}(\Omega, V):=\sup \left\{\lambda_{1}(\tilde{\Omega}, V): \tilde{\Omega} \text { open, } \Omega \subset \tilde{\Omega} \subset D\right\} .
$$

Remark 3.1. Notice that, these two definitions coincide for open sets.

Remark 3.2. The functional $\Omega \mapsto \lambda_{1}(\Omega, V)$, defined on the family of quasi-open sets, is still nonincreasing with respect to the set inclusion, that is $\lambda_{1}\left(\Omega_{2}, V\right) \leq \lambda_{1}\left(\Omega_{1}, V\right)$, whenever $\Omega_{1} \subset \Omega_{2}$.

We will show that $\lambda_{1}(\Omega, V)$ is finite and is an eigenvalue of $L$ in $\Omega$ satisfying the minimality property $(\mathrm{ii}$ ). Recall that, for a quasi-open set of finite Lebesgue measure $\Omega \subset D$, we say that $\lambda \in \mathbb{C}$ is an eigenvalue of the operator $L=-\Delta+V \cdot \nabla$ in $\Omega$ if there is an eigenfunction $u: \mathbb{R}^{d} \rightarrow \mathbb{C}$, (weak) solution to the problem

$$
-\Delta u+V \cdot \nabla u=\lambda u \quad \text { in } \quad \Omega, \quad u \in H_{0}^{1}(\Omega ; \mathbb{C}), \quad \int_{\Omega}|u|^{2} d x=1 .
$$

Let now $c>0$ be the constant from Subsection 2.2 and $L^{\prime}=L+c$. Note that $\lambda \in \mathbb{C}$ is an eigenvalue of $L$ in $\Omega$, if and only if, $\lambda+c$ is an eigenvalue of $L^{\prime}$ in $\Omega$. By the argument from Subsection 2.2. we have that the bilinear form associated to the operator $L^{\prime}$ is coercive and so, $R_{\Omega}^{L^{\prime}}$ is a compact operator on $L^{2}(D)$. In particular, the spectrum is a discrete set of eigenvalues with no accumulation points except zero and $\lambda \in \mathbb{C}$ is an eigenvalue of $L$ in the sense of (3.2) if and only if $(\lambda+c)^{-1}$ is an eigenvalue of $R_{\Omega}^{L^{\prime}}$.

The following theorem shows that most of the properties of the principal eigenvalue on an open set still hold for $\lambda_{1}(\Omega, V)$ if $\Omega \subset D$ is merely a quasi-open set.

Theorem 3.3 (Definition of the principal eigenvalue on quasi-open sets). Let $D$ be a bounded open set, $V \in L^{\infty}\left(D, \mathbb{R}^{d}\right)$ and $\Omega \subset D$ be a non-empty quasi-open set. Then

(1) $\lambda_{1}(\Omega, V)$ is well-defined that is, $\lambda_{1}(\Omega, V)<+\infty$.

(2) $\lambda_{1}(\Omega, V)$ is an eigenvalue of $L$ in $\Omega$; there is a (non-trivial) real-valued eigenfunction $u$ such that

$$
L u=\lambda_{1}(\Omega, V) u \quad \text { in } \quad \Omega, \quad u \in H_{0}^{1}(\Omega), \quad \int_{\Omega} u^{2} d x=1 .
$$

(3) If $\lambda \in \mathbb{C}$ is an eigenvalue of $L$ in $\Omega$, then $\lambda_{1}(\Omega, V) \leq \operatorname{Re}(\lambda)$.

In order to prove Theorem 3.3 we will need the following two lemmas. The key estimate for the proof of Theorem 3.3 (1) is contained in the following lemma inspired by [4. Proposition 5.1].

Lemma 3.4. Let $V \in L^{\infty}\left(D, \mathbb{R}^{d}\right)$ and $\Omega \subset D$ be an open set. Suppose that there is $\tau>0$ such that $\|V\|_{L^{\infty}(\Omega)} \leq \tau<2 \sqrt{\lambda_{1}(\Omega, V)}$. Then

$$
\lambda_{1}(\Omega, 0) \geq \lambda_{1}(\Omega, V)-\tau \sqrt{\lambda_{1}(\Omega, V)} .
$$

Proof. Let us first suppose that $\Omega$ is connected. For convenience, set $\lambda:=\lambda_{1}(\Omega, V)$. By the definition of the first eigenvalue of $-\Delta$ on domains, it is enough to find some $\phi>0$ in $\Omega$ such that $-\Delta \phi \geq(\lambda-\tau \sqrt{\lambda}) \phi$ in $\Omega$. Since $\Omega$ is an open set, from [4, Theorem 2.1], there exists a positive eigenfunction $\phi_{V}$ for the first eigenvalue of $L$ in $\Omega$, that is, $\phi_{V}>0$ in $\Omega$ and $L \phi_{V}=\lambda \phi_{V}$. Set $\phi:=\phi_{V}^{\alpha}$ for some $\alpha \in(0,1)$ to be chosen later. Then, in $\Omega$, we have

$$
\begin{aligned}
-\Delta \phi-\lambda \phi & =-\alpha\left(\Delta \phi_{V}\right) \phi_{V}^{\alpha-1}-\alpha(\alpha-1)\left|\nabla \phi_{V}\right|^{2} \phi_{V}^{\alpha-2}-\lambda \phi_{V}^{\alpha} \\
& =\left[\lambda(\alpha-1)-\alpha V \cdot \frac{\nabla \phi_{V}}{\phi_{V}}+\alpha(1-\alpha) \frac{\left|\nabla \phi_{V}\right|^{2}}{\phi_{V}^{2}}\right] \phi_{V}^{\alpha} \\
& \geq\left[\lambda(\alpha-1)-\alpha \tau \frac{\left|\nabla \phi_{V}\right|}{\phi_{V}}+\alpha(1-\alpha) \frac{\left|\nabla \phi_{V}\right|^{2}}{\phi_{V}^{2}}\right] \phi_{V}^{\alpha} .
\end{aligned}
$$


The function $x \mapsto-\alpha \tau x+\alpha(1-\alpha) x^{2}$ reaches its minimum at $x=\tau /(2(1-\alpha))$. Therefore, we get

$$
-\Delta \phi-\lambda \phi \geq\left[\lambda(\alpha-1)-\alpha \frac{\tau^{2}}{4(1-\alpha)}\right] \phi_{V}^{\alpha}=\left[\lambda(\alpha-1)-\alpha \frac{\tau^{2}}{4(1-\alpha)}\right] \phi .
$$

Since $\alpha \in(0,1)$ is arbitrary, we can choose it so that it maximizes the term in the brackets of the above estimate, that is, such that $1-\alpha=\tau /(2 \sqrt{\lambda})$. Note that, by hypothesis on $\tau$, we have $\alpha \in(0,1)$. It follows

$$
-\Delta \phi-\lambda \phi \geq\left[-\tau \sqrt{\lambda}+\frac{\tau^{2}}{4}\right] \phi \geq-\tau \sqrt{\lambda} \phi,
$$

which proves the claim in the case when $\Omega$ is connected.

In the general case, let $\left(\Omega_{n}\right)_{n \geq 1}$ be the connected components of $\Omega$. Then, for every $V$, we have

$$
\lambda_{1}(\Omega, V)=\inf _{n} \lambda_{1}\left(\Omega_{n}, V\right) .
$$

Then, we have, for all $n$,

$$
\lambda_{1}\left(\Omega_{n}, 0\right) \geq \lambda_{1}\left(\Omega_{n}, V\right)-\tau \sqrt{\lambda_{1}\left(\Omega_{n}, V\right)} \geq \lambda_{1}(\Omega, V)-\tau \sqrt{\lambda_{1}(\Omega, V)},
$$

where the last inequality is due to the fact that $x \mapsto x-\tau \sqrt{x}$ is a non-increasing function on the interval $\left[\lambda_{1}(\Omega, V),+\infty\right)$.

The next lemma is a direct consequence of the classical result [32, Theorem 3.16] on the convergence of a spectrum of closed operators with suitable properties. We will use it in the proof of Theorem 3.3 (3).

Lemma 3.5 (Convergence of the spectra). Let $H$ be a separable Hilbert space and $\left(T_{n}\right)_{n \geq 1} \in \mathcal{L}(H)$ a sequence of compact operators converging to the compact operator $T \in \mathcal{L}(H)$ in the operator norm $\|\cdot\|_{\mathcal{L}(H)}$. Suppose that $\lambda \in \mathbb{C} \backslash\{0\}$ is an (isolated) eigenvalue of $T$ and let $r>0$ be such that $B_{r}(\lambda) \cap \sigma(T)=\{\lambda\}$. Then, there is $n_{0} \geq 1$ such that for every $n \geq n_{0}$ there is an eigenvalue $\lambda_{n} \in \sigma\left(T_{n}\right) \cap B_{r / 2}(\lambda)$.

We are now in position to prove Theorem 3.3 .

Proof of Theorem 3.3. Consider a maximizing sequence $\left(\Omega_{n}\right)_{n \geq 1}$ for (3.1), that is, a sequence of open sets $\left(\Omega_{n}\right)_{n \geq 1}$ such that

$$
\lambda_{1}(\Omega, V)=\lim _{n \rightarrow \infty} \lambda_{1}\left(\Omega_{n}, V\right) \quad \text { and } \quad \Omega \subset \Omega_{n} \subset D \text { for every } n \geq 1 .
$$

We first show that we can assume that $\Omega_{n} \gamma$-converges to $\Omega$. Let $\omega_{n}$ be a sequence of open sets such that $\Omega \cup \omega_{n}$ is open and $\operatorname{cap}\left(\omega_{n}\right) \rightarrow 0$. We set $\tilde{\Omega}_{n}:=\Omega_{n} \cap\left(\Omega \cup \omega_{n}\right)=\Omega \cup\left(\omega_{n} \cap \Omega_{n}\right)$. By (3.1) and the inclusion $\Omega \subset \tilde{\Omega}_{n} \subset \Omega_{n}$ we have $\lambda_{1}\left(\Omega_{n}, V\right) \leq \lambda_{1}\left(\tilde{\Omega}_{n}, V\right) \leq \lambda_{1}(\Omega, V)$, so we get

$$
\lambda_{1}(\Omega, V)=\lim _{n \rightarrow \infty} \lambda_{1}\left(\tilde{\Omega}_{n}, V\right) \quad \text { and } \quad \Omega \subset \tilde{\Omega}_{n} \subset D \text { for every } n \geq 1 .
$$

Thus, we may consider $\tilde{\Omega}_{n}$ in place of $\Omega_{n}$ as a maximizing sequence for (3.1). Finally, as in Lemma 2.6, $\tilde{\Omega}_{n} \gamma$-converges to $\Omega$ thanks to the estimate (2.2) applied to the sets $\tilde{\Omega}_{n}$ and $\Omega_{n} \cap \omega_{n}$.

We now prove claim (1). Indeed, suppose by contradiction that

$$
\lambda_{1}(\Omega, V)=\lim _{n \rightarrow \infty} \lambda_{1}\left(\Omega_{n}, V\right)=+\infty .
$$

Then, by Lemma 3.4 we have that

$$
\lim _{n \rightarrow \infty} \lambda_{1}\left(\Omega_{n}, 0\right)=+\infty
$$

Now, since $\Omega \mapsto \lambda_{1}(\Omega, 0)$ is decreasing and $\Omega \subset \Omega_{n}$, we get that $\lambda_{1}(\Omega, 0)=+\infty$. By the variational characterization

$$
\lambda_{1}(\Omega, 0)=\min _{u \in H_{0}^{1}(\Omega) \backslash\{0\}} \frac{\int_{\Omega}|\nabla u|^{2} d x}{\int_{\Omega} u^{2} d x},
$$


we get that $H_{0}^{1}(\Omega)=\{0\}$, which implies that $\Omega=\emptyset$ (or, equivalently, cap $\Omega=0$ ), which is absurd.

We now prove (2). Let $u_{n} \in H_{0}^{1}\left(\Omega_{n}\right) \subset H_{0}^{1}(D)$ be the (normalized) eigenfunction associated to $\lambda_{1}\left(\Omega_{n}, V\right)$. Then we have

$$
L^{\prime} u_{n}=\left(\lambda_{1}\left(\Omega_{n}, V\right)+c\right) u_{n} \quad \text { in } \quad \Omega_{n}, \quad u_{n} \in H_{0}^{1}\left(\Omega_{n}\right), \quad \int_{\Omega_{n}} u_{n}^{2} d x=1 .
$$

Multiplying the above equation by $u_{n}$, integrating over $\Omega_{n}$ and using the estimate (2.3) we get

$$
\delta\left\|u_{n}\right\|_{H^{1}}^{2} \leq \lambda_{1}\left(\Omega_{n}, V\right)+c \text { for every } n \geq 1 .
$$

In particular, since $\lambda_{1}(\Omega, V)<\infty$, we get that $\left(u_{n}\right)_{n \geq 1}$ is uniformly bounded in $H_{0}^{1}(D)$ and so, up to a subsequence, we may assume that $u_{n}$ converges, weakly in $H_{0}^{1}(D)$ and strongly in $L^{2}(D)$, to a function $u \in H_{0}^{1}(D)$. Moreover, $\Omega_{n} \gamma$-converges to $\Omega$ and so, $R_{\Omega_{n}}^{L^{\prime}}$ converges in norm to $R_{\Omega}^{L^{\prime}}$. Thus,

$$
u=\lim _{n \rightarrow \infty} u_{n}=\lim _{n \rightarrow \infty}\left(\lambda_{1}\left(\Omega_{n}, V\right)+c\right) R_{\Omega_{n}}^{L^{\prime}}\left(u_{n}\right)=\left(\lambda_{1}(\Omega, V)+c\right) R_{\Omega}^{L^{\prime}}(u),
$$

which concludes the proof of (2).

Proof of (3). Suppose that $\lambda \in \mathbb{C}$ is an eigenvalue of $L$ on $\Omega$ such that $\operatorname{Re}(\lambda)<\lambda_{1}(\Omega, V)$. Then, $(\lambda+c)^{-1} \in \mathbb{C}$ is a (non-zero) eigenvalue of the compact operator $R_{\Omega}^{L^{\prime}}$. Applying Lemma 3.5. we can assume that for $n$ large enough, there is an eigenvalue $\lambda_{n}$ of $L$ on $\Omega_{n}$ such that $\operatorname{Re}\left(\lambda_{n}\right)<\lambda_{1}\left(\Omega_{n}, V\right)$, which is a contradiction with [4, Theorem 2.3] .

Remark 3.6 (On the sign of the first eigenfunction). In particular, as a consequence of the proof of Theorem 3.3 (2), there is an eigenfunction $u$ of $L$ on the quasi-open set $\Omega$, which is non-negative, being the limit of non-negative functions. We notice that $u$ does not need to be strictly positive as $\Omega$ might be disconnected.

We conclude this section with a proposition on the continuity of $\lambda_{1}(\cdot, V)$ with respect to the $\gamma$-convergence.

Proposition $3.7\left(\gamma\right.$-continuity of $\left.\lambda_{1}(\cdot, V)\right)$. Let $D \subset \mathbb{R}^{d}$ be a bounded open set, $V \in L^{\infty}\left(D ; \mathbb{R}^{d}\right)$ be a fixed vector field, and $\left(\Omega_{n}\right)_{n \geq 1} \subset D$ be a sequence of quasi-open sets that $\gamma$-converges to the quasi-open set $\Omega \subset D$. Then

$$
\lambda_{1}(\Omega, V)=\left\{\begin{array}{l}
\lim _{n \rightarrow \infty} \lambda_{1}\left(\Omega_{n}, V\right), \quad \text { if } \Omega \neq \emptyset, \\
+\infty, \quad \text { if } \Omega=\emptyset .
\end{array}\right.
$$

Proof. Let $\tau=\|V\|_{L^{\infty}(D)}$ and $\delta$ and $c$ be as in (2.3). Set $L^{\prime}=L+c$.

Suppose first that the sequence $\left(\lambda_{1}\left(\Omega_{n}, V\right)\right)_{n \geq 1}$ is bounded. Reasoning as in the proof of Theorem 3.3 (2) we get that, up to a subsequence, $\lambda_{1}\left(\Omega_{n}, V\right)$ converges to an eigenvalue $\lambda \in \mathbb{R}$ of $L$ on $\Omega$. Now, by the argument of Theorem 3.3 (3) and Lemma 3.5. we have that $\lambda$ satisfies the property (3) of Theorem 3.3, so $\lambda=\lambda_{1}(\Omega, V)$, which concludes the proof since the sequence $\left(\lambda_{1}\left(\Omega_{n}, V\right)\right)_{n \geq 1}$ is bounded.

Next, suppose that the sequence $\left(\lambda_{1}\left(\Omega_{n}, V\right)\right)_{n \geq 1}$ is unbounded. Applying Lemma 3.4, we get that, up to a subsequence, $\lim _{n \rightarrow \infty} \lambda_{1}\left(\Omega_{n}, 0\right)=+\infty$. Since $R_{\Omega_{n}}^{-\Delta}$ are self-adjoint compact operators, we get that

$$
\lim _{n \rightarrow \infty}\left\|R_{\Omega_{n}}^{-\Delta}\right\|_{\mathcal{L}\left(L^{2}(D)\right)}=\lim _{n \rightarrow \infty} \frac{1}{\lambda_{1}\left(\Omega_{n}, 0\right)}=0 .
$$

Finally, the $\gamma$-convergence gives that $R_{\Omega}^{-\Delta}(\Omega) \equiv 0$ and so, $H_{0}^{1}(\Omega)=\{0\}$ and cap $(\Omega)=0$.

Remark 3.8. In view of Proposition 3.7 we set $\lambda_{1}(\emptyset, V)=+\infty$.

Putting together Theorem 3.3 and Proposition 3.7 we obtain the following result. 
Corollary 3.9 (Equivalent definition of the principal eigenvalue on quasi-open sets). Let $\Omega$ be a bounded quasi-open set and $V \in L^{\infty}\left(\Omega ; \mathbb{R}^{d}\right)$. Then, there is an eigenvalue $\lambda_{1}(\Omega, V) \in \mathbb{R}$ of $L=-\Delta+V \cdot \nabla$ in $\Omega$ such that:

$$
\begin{aligned}
\lambda_{1}(\Omega, V) & =\min \{\operatorname{Re} \lambda: \lambda \in \mathbb{C} \text { is an eigenvalue of } L \text { on } \Omega\} \\
& =\sup \left\{\lambda_{1}(\tilde{\Omega}): \tilde{\Omega} \text { is an open set containing } \Omega\right\} \\
& =\lim _{n \rightarrow \infty} \lambda_{1}\left(\Omega_{n}, V\right), \text { where }\left(\Omega_{n}\right)_{n \geq 1} \text { is any sequence } \\
& \text { of (smooth) open sets } \gamma \text {-converging to } \Omega .
\end{aligned}
$$

Proof. The first two inequalities are due to Theorem 3.3. For the third one it is sufficient to note that for every quasi-open set $\Omega$ there is a sequence of smooth open sets $\gamma$-converging to $\Omega$ and to apply Proposition 3.7.

Remark 3.10 (Faber-Krahn with drift for quasi-open sets). As further consequence of Corollary 3.9 we can extend the Hamel-Nadirashvili-Russ inequality to the class of (bounded) quasi-open sets. Precisely, for every bounded quasi-open set $\Omega \subset \mathbb{R}^{d}$ with $|\Omega|>0$ and every $\tau>0$, we have

$$
\lambda_{1}\left(B, \tau \frac{x}{|x|}\right) \leq \lambda_{1}(\Omega, V) \quad \text { for every } \quad V \in L^{\infty}\left(\Omega ; \mathbb{R}^{d}\right) \quad \text { with } \quad\|V\|_{L^{\infty}} \leq \tau,
$$

where $B$ is the ball centered in zero of the same Lebesgue measure as $\Omega$. Indeed, let $\Omega \subset \mathbb{R}^{d}$ be a bounded quasi-open set and $V \in L^{\infty}\left(\Omega, \mathbb{R}^{d}\right)$ be such that $\|V\|_{L^{\infty}} \leq \tau$ (in what follows we assume that $V$ is extended by zero outside $\Omega$ ). Let $\left(\Omega_{n}\right)_{n \geq 1}$ be a sequence of bounded open sets which $\gamma$-converges to $\Omega$ and such that $\left|\Omega_{n}\right|$ converges to $|\Omega|$ (see Lemmalem approx qo). Denote by $B_{r_{n}}$ (resp. $B$ ) the ball centred at 0 whose Lebesgue measure is $\left|B_{r_{n}}\right|=\left|\Omega_{n}\right|$ (resp. $|B|=|\Omega|)$. Then, since $\Omega_{n}$ is an open set, we have $\lambda_{1}\left(B_{r_{n}}, \tau e_{r}\right) \leq \lambda_{1}\left(\Omega_{n}, v\right)$ thanks to [28, Remark 6.10]. Moreover, $B_{r_{n}} \gamma$-converges to $B$ (since $\left|B_{r_{n}}\right| \rightarrow|B|$ and hence $B_{r_{n}}$ converges to $B$ in the sense of Hausdorff; see [30, Proposition 3.4.2]). Therefore, Corollary [3.9] implies that $\lambda_{1}\left(B_{r_{n}}, \tau e_{r}\right)$ converges to $\lambda_{1}\left(B, \tau e_{r}\right)$ and similarly, $\lambda_{1}\left(\Omega_{n}, V\right) \rightarrow \lambda_{1}(\Omega, V)$. Passing to the limit we get (3.4).

\section{Existence of optimal domains}

In this section we prove the existence of optimal domains for the cost functional $\lambda_{1}(\Omega, V)$. We first consider the case when the drift $V$ is fixed, for which the existence follows by the result of the previous section and a classical theorem in shape optimization. The case when both the domain $\Omega$ and the drift $V$ may vary requires more careful analysis and the rest of the section is dedicated to the proof of Theorem 4.3. In the end of the section (Theorem 4.5) we also prove that a solution $(\Omega, V)$ exists also in the class of vector fields $V$ obtained as gradients of Lipschitz continuous functions.

Theorem 4.1 (Existence of optimal sets for a fixed vector field). Let $D \subset \mathbb{R}^{d}$ be a bounded open set and $V \in L^{\infty}\left(D ; \mathbb{R}^{d}\right)$. Then, for every $0<m \leq|D|$, there is an optimal domain, solution of the problem (1.8).

Proof. By Remark 3.2 and Proposition 3.7 we get that $\Omega \mapsto \lambda_{1}(\Omega, V)$ is $\gamma$-continuous and decreasing with respect to the set inclusion. The claim follows by Theorem 2.5 ,

4.1. Optimal drifts on a fixed domain. Let $\Omega \subset \mathbb{R}^{d}$ be a fixed bounded quasi-open set and $\tau>0$ be given. We consider the following variational minimization problem

$$
\min \left\{\lambda_{1}(\Omega, V): V \in L^{\infty}\left(\Omega, \mathbb{R}^{d}\right),\|V\|_{L^{\infty}} \leq \tau\right\} .
$$

Theorem 4.2 (Optimal vector field on a fixed quasi-open set). The problem (4.1) has a solution, which satisfies

$$
V_{*}(x)=-\tau \frac{\nabla u(x)}{|\nabla u(x)|} \text { if }|\nabla u(x)| \neq 0 ; \quad V_{*}(x)=0 \quad \text { if }|\nabla u(x)|=0,
$$

where $u$ is the eigenfunction of $L=-\Delta+V_{*} \cdot \nabla$ in $\Omega$, associated to the eigenvalue $\lambda_{1}\left(\Omega, V_{*}\right)$. 
Proof. Let $\left(\Omega_{n}\right)_{n \geq 1}$ be a sequence of smooth, say of class $C^{2, \alpha}$ for some $0<\alpha<1$, open sets which $\gamma$-converges to $\Omega$ (see Remark 2.6). Since $\Omega_{n}$ is smooth, we already know (see [29, theorem 1.5]) that the problem (4.1) for the fixed domain $\Omega_{n}$ has a solution $V_{n}$. Moreover, if $u_{n}$ is the associated eigenfunction of $-\Delta+V_{n} \cdot \nabla$ in $\Omega_{n}$, that is, $u_{n}$ is defined by

$$
-\Delta u_{n}+V_{n} \cdot \nabla u_{n}=\lambda_{1}\left(\Omega_{n}, V_{n}\right) u_{n} \quad \text { in } \quad \Omega_{n}, \quad u_{n} \in H_{0}^{1}\left(\Omega_{n}\right), \quad \int_{\Omega_{n}} u_{n}^{2} d x=1,
$$

then the optimal vector field $V_{n}$ is unique and is given by

$$
V_{n}(x)= \begin{cases}-\tau \frac{\nabla u_{n}(x)}{\left|\nabla u_{n}(x)\right|} & \text { if }\left|\nabla u_{n}(x)\right| \neq 0, \\ 0 & \text { if }\left|\nabla u_{n}(x)\right|=0 .\end{cases}
$$

In particular, $u_{n}$ is a solution of

$$
-\Delta u_{n}-\tau\left|\nabla u_{n}\right|=\lambda_{1}\left(\Omega_{n}, V_{n}\right) u_{n} \quad \text { in } \quad \Omega_{n}, \quad u_{n} \in H_{0}^{1}\left(\Omega_{n}\right), \quad \int_{\Omega_{n}} u_{n}^{2} d x=1 .
$$

We first claim that the sequence $\left(\lambda_{1}\left(\Omega_{n}, V_{n}\right)\right)_{n>1}$ is bounded. Indeed, by optimality of $V_{n}$, one has $\lambda_{1}\left(\Omega_{n}, V_{n}\right) \leq \lambda_{1}\left(\Omega_{n}, 0\right)$, which is nothing but the principal eigenvalue of $-\Delta$ on $\Omega_{n}$ with Dirichlet boundary condition. But since $\Omega_{n} \gamma$-converges to $\Omega$, Proposition 3.7 yields that $\lambda_{1}\left(\Omega_{n}, 0\right) \rightarrow$ $\lambda_{1}(\Omega, 0)$ so that the sequence $\left(\lambda_{1}\left(\Omega_{n}, 0\right)\right)_{n>1}$ is bounded, proving our claim.

Therefore, up to a subsequence, $\lambda_{1}\left(\Omega_{n}, V_{n}\right)$ converges to some $\lambda \in \mathbb{R}$ and $u_{n}$ has a uniformly bounded norm in $H_{0}^{1}(D)$, which yields a function $u \in H_{0}^{1}(D)$ such that, up to a subsequence,

$$
u_{n} \rightarrow u \text { weakly in } H_{0}^{1}(D) \text { and } u_{n} \rightarrow u \text { strongly in } L^{2}(D) \text {. }
$$

Since the sequence $\left|\nabla u_{n}\right|$ is bounded in $L^{2}(D)$, up to a subsequence, $-\tau\left|\nabla u_{n}\right| \rightarrow z$ weakly in $L^{2}(D)$ for some function $z \in L^{2}(D)$. Therefore, $f_{n}:=\lambda_{1}\left(\Omega_{n}, v_{n}\right) u_{n}+\tau\left|\nabla u_{n}\right|$ weakly converges in $L^{2}(D)$ to $f:=\lambda u-z$. Thanks to theorem 2.8 (applied to $-\Delta$ ), $u_{n}=R_{\Omega_{n}}^{-\Delta}\left(f_{n}\right)$ strongly converges in $L^{2}(D)$ to $R_{\Omega}^{-\Delta}(f)$. By (4.3), we have $u=R_{\Omega}^{-\Delta}(f)$ and hence $u \in H_{0}^{1}(\Omega)$. Furthermore

$$
\begin{aligned}
\int_{D}|\nabla u|^{2} d x & =\int_{D}\left(-z u+\lambda u^{2}\right) d x \\
& =\lim _{n \rightarrow+\infty} \int_{D}\left(\tau\left|\nabla u_{n}\right| u_{n}+\lambda_{1}\left(\Omega_{n}, V_{n}\right) u_{n}^{2}\right) d x=\lim _{n \rightarrow+\infty} \int_{D}\left|\nabla u_{n}\right|^{2} d x,
\end{aligned}
$$

where the first line is due to the fact that $u \in H_{0}^{1}(\Omega)$ and $-\Delta u=\lambda u-z$ in $\Omega$. This proves that $u_{n}$ converges strongly in $H^{1}(D)$ to $u$, that $\left|\nabla u_{n}\right|$ strongly converges in $L^{2}(D)$ to $|\nabla u|$, and hence that $z=-\tau|\nabla u|$. Therefore $u$ satisfies

$$
-\Delta u+V_{*} \cdot \nabla u=-\Delta u-\tau|\nabla u|=\lambda u \quad \text { in } \quad \Omega, \quad u \in H_{0}^{1}(\Omega), \quad \int_{\Omega} u^{2} d x=1,
$$

where $V_{*} \in L^{\infty}\left(D, \mathbb{R}^{d}\right)$ is given by (4.2). This shows that $\lambda$ is an eigenvalue of the operator $L=-\Delta+V_{*} \cdot \nabla$ in $\Omega$. In particular, we have $\left\|V_{*}\right\|_{\infty} \leq \tau$ and $\lambda_{1}\left(\Omega, V_{*}\right) \leq \lambda$. On the other hand, by the minimality of $V_{n}$, we have $\lambda_{1}\left(\Omega_{n}, V_{n}\right) \leq \lambda_{1}\left(\Omega_{n}, V_{*}\right)$. Hence, letting $n \rightarrow \infty$, we get that $\lambda \leq \lambda_{1}\left(\Omega, V_{*}\right)$, which yields $\lambda=\lambda_{1}\left(\Omega, V_{*}\right)$ and concludes the proof of the theorem.

4.2. Shape optimization problem over domains and vector fields. Let $D \subset \mathbb{R}^{d}$ be a bounded open set, $0<m \leq|D|$ and $\tau>0$. We consider the shape optimization problem

$$
\min \left\{\lambda_{1}(\Omega, V): \Omega \subset D \text { quasi-open, }|\Omega| \leq m,\|V\|_{L^{\infty}} \leq \tau\right\} .
$$

Theorem 4.3 (Existence of optimal sets and optimal vector fields). Let $\tau \geq 0$ and $m \in(0,|D|)$. Then the problem (4.4) has a solution $\left(\Omega^{*}, V^{*}\right)$, where $V^{*}$ is given by (4.2).

Proof. Let $\left(\Omega_{n}, V_{n}\right)$ be a minimizing sequence for (4.4) and let

$$
\underline{\lambda}:=\inf \left\{\lambda_{1}(\Omega, V): \Omega \subset D \text { quasi-open, }|\Omega| \leq m,\|V\|_{L^{\infty}} \leq \tau\right\}=\lim _{n \rightarrow \infty} \lambda_{1}\left(\Omega_{n}, V_{n}\right),
$$


Since the topology of the weak $\gamma$-convergence is compact, we can assume that, up to a subsequence, $\Omega_{n}$ weakly $\gamma$-converges to a quasi-open set $\Omega \subset D$. Then, let $\tilde{\Omega}_{n}$ be a sequence of quasi-open sets as in Lemma 2.3. Denote by $\tilde{V}_{n}$ the optimal vector field given by Theorem 4.2 on $\tilde{\Omega}_{n}$, and let $u_{n} \in H_{0}^{1}\left(\tilde{\Omega}_{n}\right)$ be a solution of

$$
-\Delta u_{n}+\tilde{V}_{n} \cdot \nabla u_{n}=\lambda_{1}\left(\tilde{\Omega}_{n}, \tilde{V}_{n}\right) u_{n} \quad \text { in } \quad \tilde{\Omega}_{n}, \quad u_{n} \in H_{0}^{1}\left(\tilde{\Omega}_{n}\right), \quad \int_{D} u_{n}^{2} d x=1 .
$$

By the minimality of $\tilde{V}_{n}$ and the inclusion $\Omega_{n} \subset \tilde{\Omega}_{n}$, we have

$$
0<\lambda_{1}\left(\tilde{\Omega}_{n}, \tilde{V}_{n}\right) \leq \lambda_{1}\left(\tilde{\Omega}_{n}, V_{n}\right) \leq \lambda_{1}\left(\Omega_{n}, V_{n}\right) \quad \text { for every } n \geq 1 .
$$

Therefore, up to a subsequence, $\lambda_{1}\left(\tilde{\Omega}_{n}, \tilde{V}_{n}\right)$ converges to some $\tilde{\lambda}$ such that $\tilde{\lambda} \leq \underline{\lambda}$. In particular, $\left(u_{n}\right)_{n \geq 1}$ is uniformly bounded in $H_{0}^{1}(D)$ and so, up to a subsequence, $u_{n}$ weakly converges in $H_{0}^{1}(D)$ to some $u \in H_{0}^{1}(D)$. Now, since $\tilde{\Omega}_{n} \gamma$-converges to $\Omega$, we can argue as in the end of the proof of Theorem 4.2 to conclude that the convergence of $u_{n}$ to $u$ is strong in $H^{1}(D)$. This yields that $u$ is not identically zero and satisfies

$$
-\Delta u+V \cdot \nabla u=\Delta u-\tau|\nabla u|=\tilde{\lambda} u \quad \text { in } \quad \Omega, \quad u \in H_{0}^{1}(\Omega), \quad \int_{D} u^{2} d x=1,
$$

where $V \in L^{\infty}\left(D, \mathbb{R}^{d}\right)$ is given by (4.2). Furthermore, thanks to Lemma 2.4, we have that $|\Omega| \leq m$. Hence, $\underline{\lambda} \leq \tilde{\lambda}$. Thus, we get that $\tilde{\lambda}=\underline{\lambda}$ and hence that $\underline{\lambda}=\lambda_{1}(\Omega, V)$, which proves that the couple $(\Omega, V)$ is a solution of (4.4).

Remark 4.4. If the box $D$ contains a ball $B \subset D$ such that $|B|=m$, then by Remark [3.10 a solution of (4.4) is given by $\lambda_{1}\left(B, \frac{\tau x}{|x|}\right)$.

We now consider a shape optimization problem in the more restrictive class of couples $(\Omega, V)$, in which the vector field $V$ is a gradient of a Lipschitz function. Precisely, given a bounded open set $D \subset \mathbb{R}^{d}, \tau \geq 0$ and $m \in(0,|D|)$, we consider the shape optimization problem

$$
\min \left\{\lambda_{1}(\Omega, \nabla \Phi): \Omega \subset D \text { quasi-open, } \Phi \in W^{1, \infty}(D),|\Omega| \leq m,\|\nabla \Phi\|_{L^{\infty}} \leq \tau\right\} .
$$

In this case the argument from Theorem 4.3 does not apply since the optimal vector field from Theorem 4.2 may not be the gradient of a Lipschitz function. On the other hand, the functional $\lambda_{1}(\Omega, \nabla \Phi)$ is variational so we can use a more direct approach. Indeed, for every $\lambda \in \mathbb{R}$ and $u \in H_{0}^{1}(\Omega)$ we have

$$
-\Delta u+\nabla \Phi \cdot \nabla u=\lambda u \quad \text { in } \quad \Omega \quad \Leftrightarrow \quad-\operatorname{div}\left(e^{-\Phi} \nabla u\right)=\lambda e^{-\Phi} u \quad \text { in } \quad \Omega,
$$

and since the operator $A=-\operatorname{div}\left(e^{-\Phi} \nabla \cdot\right)$ is self-adjoint, we get that

$$
\lambda_{1}(\Omega, \nabla \Phi)=\min _{u \in H_{0}^{1}(\Omega) \backslash\{0\}} \frac{\int_{D} e^{-\Phi}|\nabla u|^{2} d x}{\int_{D} e^{-\Phi} u^{2} d x} .
$$

Theorem 4.5 (Existence of optimal sets and optimal potentials). Let $D \subset \mathbb{R}^{d}$ be a bounded open set, $\tau \geq 0$ and $m \in(0,|D|)$. Then the problem (4.5) has a solution.

Proof. Suppose that $\left(\Omega_{n}, \Phi_{n}\right)$ is a minimizing sequence for (4.5) and let $\lambda_{n}=\lambda_{1}\left(\Omega_{n}, \nabla \Phi_{n}\right)$. Given $x_{0} \in D$, we may suppose that $\Phi_{n}\left(x_{0}\right)=0$ for every $n \geq 1$. Thus, up to a subsequence, $\Phi_{n}$ converges uniformly in $\bar{D}$ to a function $\Phi \in W^{1, \infty}(D)$ such that $\Phi\left(x_{0}\right)=0$ and $\|\nabla \Phi\|_{L^{\infty}} \leq \tau$. Let $u_{n}$ be the solution of

$$
-\Delta u_{n}+\nabla \Phi_{n} \cdot \nabla u_{n}=\lambda_{n} u_{n} \quad \text { in } \quad \Omega_{n}, \quad u_{n} \in H_{0}^{1}\left(\Omega_{n}\right), \quad \int_{D} u_{n}^{2} d x=1 .
$$

Then, $u_{n}$ is uniformly bounded in $H_{0}^{1}(D)$ an so, up to a subsequence, $u_{n}$ converges weakly in $H_{0}^{1}(D)$ and strongly in $L^{2}(D)$ to a function $u \in H_{0}^{1}(D)$. Thus, we have

$$
\int_{D} e^{-\Phi} u^{2} d x=\lim _{n \rightarrow \infty} \int_{D} e^{-\Phi_{n}} u_{n}^{2} d x \quad \text { and } \quad \int_{D} e^{-\Phi}|\nabla u|^{2} d x \leq \liminf _{n \rightarrow \infty} \int_{D} e^{-\Phi_{n}}\left|\nabla u_{n}\right|^{2} d x
$$


Now, choosing $\Omega:=\{u>0\}$ and applying (4.6), we get

$$
\lambda_{1}(\Omega, \Phi) \leq \frac{\int_{D} e^{-\Phi}|\nabla u|^{2} d x}{\int_{D} e^{-\Phi} u^{2} d x} \leq \liminf _{n \rightarrow \infty} \frac{\int_{D} e^{-\Phi_{n}}\left|\nabla u_{n}\right|^{2} d x}{\int_{D} e^{-\Phi_{n}} u_{n}^{2} d x}=\liminf _{n \rightarrow \infty} \lambda_{1}\left(\Omega_{n}, \Phi_{n}\right) .
$$

Now, in order to conclude, it is sufficient to notice that by choosing a subsequence, we may assume that $u_{n}$ converges to $u$ pointwise a.e., so we get

$$
|\Omega|=|\{u>0\}| \leq \liminf _{n \rightarrow \infty}\left|\left\{u_{n}>0\right\}\right| \leq \liminf _{n \rightarrow \infty}\left|\Omega_{n}\right| \leq m,
$$

which proves that $(\Omega, \Phi)$ is a solution of (4.5).

\section{Regularity of the optimal Sets}

In this section we prove Theorem 1.5. We prove the regularity of the boundary $\partial \Omega$ of the optimal sets $\Omega$ from Theorem 1.5. We only consider the case $V=\nabla \Phi$, with $\Phi \in W^{1, \infty}(D)$, since in this case the optimization problem (1.8) is equivalent to a free boundary problem for the first eigenfunction $u$ on the optimal set $\Omega$. The regularity for a generic vector field $V \in L^{\infty}(D)$ remains an open problem essentially due to the lack of variational characterization of the eigenvalue $\lambda_{1}(\Omega, V)$. We start with the following lemma.

Lemma 5.1 (Reduction to a free boundary problem). Let $D \subset \mathbb{R}^{d}$ be a bounded open set, $0<m<|D|, \tau>0, \Phi \in W^{1, \infty}(D)$, with $\|\nabla \Phi\|_{L^{\infty}} \leq \tau$, and $V=\nabla \Phi$. Suppose that the quasiopen set $\Omega \subset D$ is a solution of (1.8). Then every corresponding first eigenfunction $u_{\Omega}$ of the operator $-\Delta+V \cdot \nabla$ on $\Omega$ is a solution to the variational problem

$$
\lambda_{m}:=\min \left\{\int_{D}|\nabla u|^{2} e^{-\Phi} d x: u \in H_{0}^{1}(D),|\{u \neq 0\}| \leq m, \int_{D} e^{-\Phi} u^{2} d x=1\right\} .
$$

Conversely, if $u$ is a solution of (5.1), then the quasi-open set $\{u \neq 0\}$ is a solution of (1.8).

Proof. The proof is a straightforward consequence of the variational formula (4.6).

Remark 5.2. It turns out that if $u$ is a solution of (5.1), then $u \geq 0$ in $D$ (see Lemma 5.8 below).

The rest of this section is dedicated to the regularity of the free boundary $\partial \Omega_{u} \cap D$ and of the whole boundary $\partial \Omega_{u}$ if $D$ is smooth, of a solution $u$ of (5.1), where we recall that, for any function $v \in H_{0}^{1}(D)$ we denote by $\Omega_{v}$ the (quasi-open) set $\{v>0\}$.

This section is organized as follows.

In Subsection 5.1 we prove that the solutions of (5.1) are bounded. This is important due to the fact that $u$ solves the equation

$$
-\operatorname{div}\left(e^{-\Phi} \nabla u\right)=\lambda_{1}\left(\Omega_{u}, \nabla \Phi\right) e^{-\Phi} u \quad \text { in } \quad \Omega_{u},
$$

and in the rest of the section we will often use the fact that the right-hand side is bounded.

In Subsection 5.2, we prove that the solution $u$ is essentially a subharmonic function on $D$ with respect to the operator $\operatorname{div}\left(e^{-\Phi} \nabla\right)$ (see Lemma 5.5). In particular, this implies that $u$ and the set $\Omega_{u}$ are well-defined everywhere (not just up to a set of measure zero). The free boundary is thus defined as the topological boundary of the set $\Omega_{u}$. In the same subsection, in Lemma 5.8, we prove that the measure constraint $|\{u>0\}| \leq m$ is saturated, that is, $\left|\Omega_{u}\right|=m$. This proves Theorem 1.5 (3).

In Subsection 5.3 we get rid of the integral constraint $\int e^{-\Phi} u^{2} d x=1$ and we rewrite the problem (5.1) in terms of the functional

$$
J(u)=\int_{D}\left(|\nabla u|^{2}-\lambda_{m} u^{2}\right) e^{-\Phi} d x .
$$

In Subsection 5.4, we write the Euler-Lagrange equation that arises in the minimization of the functional $J$ under the measure constraint $\left|\Omega_{u}\right| \leq m$. We consdider only internal variations, that is, test functions of the form $\tilde{u}(x)=u(x+t \xi(x))$ for smooth vector fields $\xi$. In Subsection 5.5. 
we prove that at small scales $u$ is a solution (in the sense of (1.12) ) to the minimization problem for the functional $v \mapsto J(v)+\Lambda_{u}\left|\Omega_{v}\right|$, where $\Lambda_{u}$ is the Lagrange multiplier from Subsection 5.4.

In Subsection 5.6 and Subsection [5.7, we use the result from Subsection 5.5 to prove that the solutions of (5.1) are Lipschitz continuous and non-degenerate at the free boundary; we also prove that the set $\Omega_{u}$ has finite perimeter. This proves Theorem 1.5 (1) and (2).

Subsection 5.8 is dedicated to the compactness of the blow-up sequences and the optimality of the blow-up limits. In Subsection [5.10 we prove a (quasi-)monotoncity formula for (a variant of) the Weiss' boundary adjusted energy. As a consequence, we obtain that the blow-up limits are one-homogeneaous.

In Subsection 5.9, we prove that the solution $u$ satisfies the optimality condition $|\nabla u|=\sqrt{\Lambda_{u} e^{\Phi}}$ on the free boundary $\partial \Omega_{u} \cap D$ in viscosity sense, we deduce the regularity of the regular part $\operatorname{Reg}\left(\partial \Omega_{u}\right)$ and we show that the remaining singular set has zero $(d-1)$-dimensional Hausdorff measure. In this subsection, we complete the proof of Theorem 1.5 (4), (6) and (7). Finally, in Subsection 5.10, we give some further estimates on the Hausdorff dimension of the singular set, which complete the proof of Theorem 1.5 (5).

5.1. Boundedness of the eigenfunctions. In this subsection we give a bound on the $L^{\infty}$ norm of the eigenfunctions on generic bounded quasi-open sets. We first prove that if $u$ is a solution of a PDE with sufficiently integrable right-hand side, then $u$ is bounded. Then we use and iterate an interpolation argument to improve the integrability of the eigenfunctions.

Lemma 5.3 (Boundedness of the solutions of PDEs on quasi-open sets). Let $D \subset \mathbb{R}^{d}$ be a bounded open set, $\Omega \subset D$ be a quasi-open set and $\Phi \in W^{1, \infty}(D)$. Let $f \in L^{p}(D)$ for some $p \in(d / 2,+\infty]$ and let $u \in H_{0}^{1}(\Omega)$ be the solution of

$$
-\operatorname{div}\left(e^{-\Phi} \nabla u\right)=f \quad \text { in } \quad \Omega, \quad u \in H_{0}^{1}(\Omega) .
$$

Then, there is a dimensional constant $C_{d}$ such that

$$
\|u\|_{L^{\infty}} \leq \frac{C_{d} e^{\max \Phi}}{2 / d-1 / p}|\Omega|^{2 / d-1 / p}\|f\|_{L^{p}}
$$

where $\max \Phi=\|\Phi\|_{L^{\infty}(D)}$.

Proof. We first assume that $f$ is a non-negative function. We notice that $u \geq 0$ on $\Omega$ and that $u$ is a minimum in $H_{0}^{1}(\Omega)$ of the functional

$$
J(u):=\frac{1}{2} \int_{\Omega} e^{-\Phi}|\nabla u|^{2} d x-\int_{\Omega} f u d x
$$

The rest of the proof follows precisely as in [39, Lemma 3.51]. For every $0<t<\|u\|_{L^{\infty}}$ and $\varepsilon>0$, we consider the test function $u_{t, \varepsilon}=u \wedge t+(u-t-\varepsilon)_{+}$. The inequality $J(u) \leq J\left(u_{t, \varepsilon}\right)$ gives that

$$
\frac{1}{2} \int_{\{t<u \leq t+\varepsilon\}} e^{-\Phi}|\nabla u|^{2} d x \leq \int_{\mathbb{R}^{d}} f\left(u-u_{t, \varepsilon}\right) d x \leq \varepsilon \int_{\{u>t\}} f d x \leq \varepsilon\|f\|_{L^{p}}|\{u>t\}|^{\frac{p-1}{p}},
$$

and, using the co-area formula and passing to the limit as $\varepsilon \rightarrow 0$, we get

$$
\int_{\{u=t\}}|\nabla u| d \mathcal{H}^{d-1} \leq 2 e^{\max \Phi}\|f\|_{L^{p}}|\{u>t\}|^{\frac{p-1}{p}} .
$$

Now, setting $\varphi(t):=|\{u>t\}|$ and using the co-area formula again as well as the Cauchy-Schwarz inequality, we obtain

$$
\varphi^{\prime}(t)=-\int_{\{u=t\}} \frac{1}{|\nabla u|} d \mathcal{H}^{d-1} \leq-\left(\int_{\{u=t\}}|\nabla u| d \mathcal{H}^{d-1}\right)^{-1} \operatorname{Per}(\{u>t\})^{2},
$$


which, together with the isoperimetric inequality $|\{u>t\}|^{\frac{d-1}{d}} \leq C_{d} \operatorname{Per}(\{u>t\})$ and (5.3), gives

$$
\varphi^{\prime}(t) \leq-\frac{C_{d}}{e^{\max \Phi}\|f\|_{L^{p}}} \varphi(t)^{\frac{d-2}{d}+\frac{1}{p}},
$$

where we recall that the dimensional constant $C_{d}$ may change from line to line.

Setting $\alpha=\frac{d-2}{d}+\frac{1}{p}<1$ and $C=C_{d}\|f\|_{L^{p}}^{-1} e^{-\max \Phi}$, we have $\varphi^{\prime} \leq-C \varphi^{\alpha}$. If

$$
t_{\max }:=\sup \{t>0 ; \varphi(s)>0 \text { for all } s \in[0, t)\} \leq+\infty,
$$

then $\varphi^{\prime}(t) \varphi(t)^{-\alpha} \leq-C$ for all $t \in\left[0, t_{\max }\right)$, so that

$$
0 \leq \varphi(t) \leq\left(|\Omega|^{1-\alpha}-(1-\alpha) C t\right)^{\frac{1}{1-\alpha}} \quad \text { for all } \quad t \in\left[0, t_{\max }\right) .
$$

This shows that $t_{\max }<+\infty$ and that

$$
\|u\|_{L^{\infty}} \leq t_{\max } \leq \frac{1}{C} \frac{|\Omega|^{2 / d-1 / p}}{2 / d-1 / p},
$$

which concludes the proof when $f$ is non-negative. For a general function $f$, the proof now follows by applying the estimate in Lemma 5.3 to both the positive and the negative parts of $f$.

Lemma 5.4 (Boundedness of the eigenfunctions). Let $D \subset \mathbb{R}^{d}$ be a bounded open set, $\Omega \subset D$ be a quasi-open set, $\Phi \in W^{1, \infty}(D)$ and $V=\nabla \Phi$. Let $R: L^{2}(\Omega) \rightarrow L^{2}(\Omega)$ be the resolvent operator of $-\Delta+V \cdot \nabla$ on $\Omega$. Then, there are constants $n \in \mathbb{N}$, depending only on $d$, and $C \in \mathbb{R}$, depending on $d,|\Omega|$ and $\|\Phi\|_{L^{\infty}}$, such that

$$
R^{n}\left(L^{2}(\Omega)\right) \subset L^{\infty}(\Omega) \quad \text { and } \quad\left\|R^{n}\right\|_{\mathcal{L}\left(L^{2}(\Omega) ; L^{\infty}(\Omega)\right)} \leq C .
$$

In particular, if $u$ is a first eigenfunction of $-\Delta+V \cdot \nabla$ on $\Omega$ normalized by $\|u\|_{L^{2}}=1$, then $u \in L^{\infty}(\Omega)$ and

$$
\|u\|_{L^{\infty}} \leq C \lambda_{1}^{n}(\Omega, V) .
$$

Proof. Let us first notice that if $d \leq 3$, then $d / 2<2$ and so, taking $n=1$, the claim follows directly by Lemma 5.3. If $d>3$, then setting $2^{*}=\frac{2 d}{d-2}$, we have

$$
R: L^{2}(\Omega) \rightarrow L^{2^{*}}(\Omega) \quad \text { and } \quad R: L^{d}(\Omega) \rightarrow L^{\infty}(\Omega) .
$$

Thus, interpolating between 2 and $d$, we get

$$
\|R\|_{\mathcal{L}\left(L^{p} ; L^{q}\right)} \leq C, \quad \text { where } \quad p \in[2, d] \quad \text { and } \quad q=\frac{p d}{d-p} \geq \frac{p d}{d-2},
$$

where $C$ depends only on $d,|\Omega|$ and $\|\Phi\|_{L^{\infty}}$. Now, it is sufficient to notice that $R^{k} \in \mathcal{L}\left(L^{2} ; L^{q_{k}}\right)$, where $q_{k}=2\left(\frac{d}{d-2}\right)^{k}$. For $k$ big enough we have that $q_{k}>d / 2$ and so, $R^{k+1} \in \mathcal{L}\left(L^{2} ; L^{\infty}\right)$, which proves the first part of the claim. Finally, in order to get the estimate on $u$, it is sufficient to notice that $R(u)=\lambda_{1}^{-1}(\Omega, V) u$ and $R^{n}(u)=\lambda_{1}^{-n}(\Omega, V) u$.

5.2. Pointwise definition of the solutions. When we deal with Sobolev functions we usually reason up to a choice of certain representative of the function. Even if this representative is defined quasi-everywhere, there still might be a set of zero capacity where the function is not defined. Of course, this interferes with the notion of a free boundary in the sense that we cannot just consider the topological boundary of $\Omega_{u}$ without specifying the representative of $u$ that we work with. Fortunately, the eigenfunctions of the quasi-open sets are defined pointwise everywhere, that is every point is a Lebesgue point.

Lemma 5.5 (Subharmonicity and a mean-value formula for positive solutions of PDEs). Let $D \subset \mathbb{R}^{d}$ be a bounded open set, $\Omega \subset D$ a quasi-open set and $\Phi \in W^{1, \infty}(D)$ a given Lipschitz function. Let $f \in L^{\infty}(D)$ and $u \geq 0$ be a solution to the problem (5.2) in $\Omega$.

(1) Then, $\operatorname{div}\left(e^{-\Phi} \nabla u\right)+f \geq 0$ in $\mathbb{R}^{d}$, in the sense of distributions. In particular, $\operatorname{div}\left(e^{-\Phi} \nabla u\right)$ is a (signed) Radon measure on $\mathbb{R}^{d}$. 
(2) For any $x_{0} \in \mathbb{R}^{d}$, we can define the value of $u$ at $x_{0}$ by

$$
u\left(x_{0}\right)=\lim _{r \rightarrow 0} f_{\partial B_{r}\left(x_{0}\right)} u(x) d \mathcal{H}^{d-1}(x)=\lim _{r \rightarrow 0} f_{B_{r}\left(x_{0}\right)} u(x) d x .
$$

Moreover, we have the identity

$$
\begin{aligned}
f_{\partial B_{r}\left(x_{0}\right)} u e^{-\Phi} d \mathcal{H}^{d-1}-u\left(x_{0}\right) e^{-\Phi\left(x_{0}\right)}=\frac{1}{d \omega_{d}} & \int_{0}^{r} s^{1-d} \operatorname{div}\left(e^{-\Phi} \nabla u\right)\left(B_{s}\left(x_{0}\right)\right) d s \\
& -\frac{1}{d \omega_{d}} \int_{0}^{r} s^{1-d} d s \int_{\partial B_{s}}(\nabla \Phi \cdot \nu) u e^{-\Phi} d \mathcal{H}^{d-1},
\end{aligned}
$$

where $\nu$ denotes the normal to $\partial B_{\text {s }}$ pointing outwards.

(3) Let $x_{0} \in \mathbb{R}^{d}$ and $R>0$. Suppose that there is a constant $C>0$ such that

$$
\left|\operatorname{div}\left(e^{-\Phi} \nabla u\right)\left(B_{r}\left(x_{0}\right)\right)\right| \leq C r^{d-1} \quad \text { for every } \quad 0<r \leq R .
$$

Then we have the estimate

$$
\begin{gathered}
\left|u\left(x_{0}\right)-f_{\partial B_{r}\left(x_{0}\right)} u d \mathcal{H}^{d-1}\right| \leq e^{M_{\Phi}}\left(\frac{C}{d \omega_{d}}+2 L_{\Phi} M_{u} e^{M_{\Phi}}\right) r \quad \text { for every } \quad 0<r \leq R, \\
\text { where } L_{\Phi}:=\|\nabla \Phi\|_{L^{\infty}(D)}, M_{u}:=\|u\|_{L^{\infty}(D)} \text { and } M_{\Phi}:=\|\Phi\|_{L^{\infty}(D)} .
\end{gathered}
$$

Proof. (1) For $n \in \mathbb{N}$ define $p_{n}: \mathbb{R} \rightarrow \mathbb{R}$ by

$$
p_{n}(s)=0, \text { for } s \leq 0 ; \quad p_{n}(s)=n s, \text { for } s \in[0,1 / n] ; \quad p_{n}(s)=1, \text { for } s \geq 1 / n .
$$

Since $p_{n}$ is Lipschitz continuous, we have $p_{n}(u) \in H_{0}^{1}(\Omega)$ and $\nabla p_{n}(u)=p_{n}^{\prime}(u) \nabla u$. Let $\varphi \in$ $C_{0}^{\infty}(D), \varphi \geq 0$ in $D$. Using $\varphi p_{n}(u)$ as a test function in (5.2), we get

$$
\int_{D} p_{n}(u) \nabla u \cdot \nabla \varphi e^{-\Phi} d x \leq \int_{D}\left(p_{n}(u) \nabla u \cdot \nabla \varphi+\varphi p_{n}^{\prime}(u)|\nabla u|^{2}\right) e^{-\Phi} d x=\int_{D} f \varphi p_{n}(u) d x .
$$

which, letting $n \rightarrow \infty$, gives the first claim.

In order to prove (2), we suppose that $x_{0}=0$ and we calculate

$$
\begin{aligned}
\frac{d}{d s} f_{\partial B_{s}} u e^{-\Phi} d \mathcal{H}^{d-1} & =\frac{d}{d s} f_{\partial B_{1}} u(s \xi) e^{-\Phi(s \xi)} d \mathcal{H}^{d-1} \\
& =f_{\partial B_{1}}[\xi \cdot \nabla u(s \xi)-u(s \xi) \xi \cdot \nabla \Phi(s \xi)] e^{-\Phi(s \xi)} d \mathcal{H}^{d-1} \\
& =\frac{s^{1-d}}{d \omega_{d}} \operatorname{div}\left(e^{-\Phi} \nabla u\right)\left(B_{s}\right)-\frac{s^{1-d}}{d \omega_{d}} \int_{\partial B_{s}}(\nabla \Phi \cdot \nu) u e^{-\Phi} d \mathcal{H}^{d-1} .
\end{aligned}
$$

Then, integrating from $\rho$ to $r(\rho<r)$, using the inequality from (1) and the fact that $u \in L^{\infty}(D)$ by Lemma 5.4, we get

$$
\begin{aligned}
f_{\partial B_{r}} u e^{-\Phi} d \mathcal{H}^{d-1}-f_{\partial B_{\rho}} u e^{-\Phi} d \mathcal{H}^{d-1} & =\frac{1}{d \omega_{d}} \int_{\rho}^{r} s^{1-d} \operatorname{div}\left(e^{-\Phi} \nabla u\right)\left(B_{s}\left(x_{0}\right)\right) d s \\
& -\frac{1}{d \omega_{d}} \int_{\rho}^{r} s^{1-d} d s \int_{\partial B_{s}}(\nabla \Phi \cdot \nu) u e^{-\Phi} d \mathcal{H}^{d-1} \\
& \geq-\frac{1}{2 d}\|f\|_{L^{\infty}}\left(r^{2}-\rho^{2}\right)-e^{-\min \Phi}\|u\|_{L^{\infty}}\|\nabla \Phi\|_{L^{\infty}}(r-\rho) \\
& :=-A\left(r^{2}-\rho^{2}\right)-B(r-\rho),
\end{aligned}
$$

where $A, B>0$. This shows that the function $r \mapsto f_{\partial B_{r}} u e^{-\Phi} d \mathcal{H}^{d-1}+A r^{2}+B r$ is non-decreasing. In particular, the limit $\ell\left(x_{0}\right)=\lim _{r \rightarrow 0} f_{\partial B_{r}\left(x_{0}\right)} u e^{-\Phi} d \mathcal{H}^{d-1}$ exists and we set $u\left(x_{0}\right):=e^{\Phi\left(x_{0}\right)} \ell\left(x_{0}\right)$. 
Now, (5.5) follows by letting $\rho \rightarrow 0$ in (5.8). Finally, in order to prove the claim (3), we notice that (5.5) implies

$$
\left|f_{\partial B_{r}\left(x_{0}\right)} u e^{-\Phi} d \mathcal{H}^{d-1}-u\left(x_{0}\right) e^{-\Phi\left(x_{0}\right)}\right| \leq\left(\frac{C}{d \omega_{d}}+L_{\Phi} M_{u} e^{M_{\Phi}}\right) r \quad \text { for every } \quad 0<r \leq R,
$$

Now, by the triangular inequality we have

$$
\begin{aligned}
\left|f_{\partial B_{r}\left(x_{0}\right)} u d \mathcal{H}^{d-1}-u\left(x_{0}\right)\right|= & e^{\Phi\left(x_{0}\right)}\left|f_{\partial B_{r}\left(x_{0}\right)} u e^{-\Phi\left(x_{0}\right)} d \mathcal{H}^{d-1}-u\left(x_{0}\right) e^{-\Phi\left(x_{0}\right)}\right| \\
\leq & e^{\Phi\left(x_{0}\right)}\left|f_{\partial B_{r}\left(x_{0}\right)} u e^{-\Phi} d \mathcal{H}^{d-1}-u\left(x_{0}\right) e^{-\Phi\left(x_{0}\right)}\right| \\
& \quad+e^{\Phi\left(x_{0}\right)} f_{\partial B_{r}\left(x_{0}\right)} u\left|e^{-\Phi\left(x_{0}\right)}-e^{-\Phi(x)}\right| d \mathcal{H}^{d-1}
\end{aligned}
$$

Thus, the claim follows since, by the Lipschitz continuity of $\Phi$, we have that for every $x \in \partial B_{r}\left(x_{0}\right)$,

$$
\left|e^{-\Phi\left(x_{0}\right)}-e^{-\Phi(x)}\right| \leq e^{\|\Phi\|_{L^{\infty}(D)}}\|\nabla \Phi\|_{L^{\infty}(D)}\left|x-x_{0}\right| .
$$

(5.7) is a direct consequence of (5.5).

As a direct consequence of Lemma 5.5 and (5.5) we get the following strong maximum principle.

Lemma 5.6 (Strong maximum principle). Let $D \subset \mathbb{R}^{d}$ be an open connected set and $u \in H_{0}^{1}(D)$ satisfy $u \geq 0$. Assume that $\operatorname{div}\left(e^{-\Phi} \nabla u\right) \in L^{\infty}(D)$ satisfies $\operatorname{div}\left(e^{-\Phi} \nabla u\right) \leq 0$. Then, if $u$ is not identically vanishing in $D$, then $u$ is strictly positive in $D$.

Proof. Set $A:=\left\{x_{0} \in D ; u\left(x_{0}\right)=0\right\}$. If $x_{0} \in A$, then (5.5) implies that $u(x)=0$ for almost every $x \in B_{r}\left(x_{0}\right)$ whenever $B_{r}\left(x_{0}\right) \subset D$. Therefore, for all $x \in B_{r}\left(x_{0}\right)$, since $x$ is a Lebesgue point for $u, u(x)=0$. Thus, $A$ is open.

Consider now a sequence $\left(x_{n}\right)_{n \geq 1} \in A$ converging to $x_{0} \in D$. For some $n$ large enough, there exists a ball $B_{r}\left(x_{n}\right) \subset D$ containing $x_{0}$. Since $u$ vanishes everywhere in $B_{r}\left(x_{n}\right), u\left(x_{0}\right)=0$, which proves that $A$ is closed in $D$. We conclude by the connectedness of $D$.

A consequence of Lemma 5.5 is the fact that the set $\Omega_{u}=\{u>0\}$ and the (topological) free boundary $\partial \Omega_{u} \cap D$ are well defined. Below we prove that the topological boundary coincides with the measure theoretic one.

Lemma 5.7 (The topological boundary coincides with the measure-theoretic one). Let $u \in$ $H_{0}^{1}(D), u \geq 0$ in $D$, be a solution of (5.1), $x_{0} \in \partial \Omega_{u}$ and let $r>0$ be such that $D_{r}\left(x_{0}\right):=$ $B_{r}\left(x_{0}\right) \cap D$ is connected. Then we have $0<\left|\Omega_{u} \cap B_{r}\left(x_{0}\right)\right|$. Moreover, if $x_{0} \in \partial \Omega_{u} \cap D$, we have $\left|\Omega_{u} \cap B_{r}\left(x_{0}\right)\right|<\left|D_{r}\left(x_{0}\right)\right|$.

Proof. In order to prove the first inequality, suppose that $x_{0} \in \partial \Omega_{u}$ and $\left|B_{r}\left(x_{0}\right) \cap \Omega_{u}\right|=0$ for some $r>0$. Since every point $x \in B_{r}\left(x_{0}\right)$ is a Lebesgue point for $u$ and $u=0$ almost everywhere in $B_{r}\left(x_{0}\right)$ we have that $u \equiv 0$ in $B_{r}\left(x_{0}\right)$, but this contradicts the fact that $x_{0} \in \partial \Omega_{u}$.

In order to show the second inequality, we assume by contradiction that $\left|\Omega_{u} \cap B_{r}\left(x_{0}\right)\right|=\left|D_{r}\left(x_{0}\right)\right|$ for some $r>0$. We claim that $u$ is a solution of

$$
-\operatorname{div}\left(e^{-\Phi} \nabla u\right)=\lambda_{m} u e^{-\Phi} \quad \text { in } \quad D_{r}\left(x_{0}\right), \quad \text { where } \quad \lambda_{m}:=\int_{D}|\nabla u|^{2} e^{-\Phi} d x .
$$

Indeed, let $v$ be the solution of

$$
-\operatorname{div}\left(e^{-\Phi} \nabla v\right)=\lambda_{m} u e^{-\Phi} \quad \text { in } \quad D_{r}\left(x_{0}\right), \quad v=u \quad \text { in } \quad D \backslash B_{r}\left(x_{0}\right) .
$$

Then Lemma 5.6 implies that $v>0$ in $D_{r}\left(x_{0}\right)$. Since $\left|\Omega_{v}\right|=\left|\Omega_{u}\right|$, the optimality of $u$ gives

$$
\frac{\int_{D}|\nabla v|^{2} e^{-\Phi} d x}{\int_{D} v^{2} e^{-\Phi} d x} \geq \int_{D}|\nabla u|^{2} e^{-\Phi} d x=\frac{\int_{D}|\nabla u|^{2} e^{-\Phi} d x}{\int_{D} v^{2} e^{-\Phi} d x}+\lambda_{m}\left(1-\frac{\int_{D} u^{2} e^{-\Phi} d x}{\int_{D} v^{2} e^{-\Phi} d x}\right),
$$


which implies

$$
0 \geq \int_{D}\left(|\nabla u|^{2}-|\nabla v|^{2}\right) e^{-\Phi} d x+\lambda_{m} \int_{D}\left(v^{2}-u^{2}\right) e^{-\Phi} d x=\int_{D}\left(|\nabla(u-v)|^{2}+\lambda_{m}(u-v)^{2}\right) e^{-\Phi} d x
$$

where the last equality follows by the definition of $v$ and the fact that $v-u \in H_{0}^{1}\left(D_{r}\left(x_{0}\right)\right)$. This implies that $u=v$ almost everywhere and hence, by Lemma 5.5, that $u=v$ everywhere. Therefore, we have $u>0$ in $B_{r}\left(x_{0}\right)$, which is in contradiction with $x_{0} \in \partial \Omega_{u} \cap D$.

Lemma 5.8 (Saturation of the constraint). Let $D \subset \mathbb{R}^{d}$ be an open connected set, $\Phi \in W^{1, \infty}(D)$, $m$ and $\tau$ be as in Lemma 5.1. Then every solution $u$ of (5.1) is such that $u \geq 0$ on $D$ and $\left|\Omega_{u}\right|=m$ (up to a change of sign). In particular, every solution $\Omega$ of (1.8) is such that $|\Omega|=m$.

Proof. Let $u$ be a solution of (5.1) and set

$$
u_{1}=\frac{u_{+}}{\left(\int_{D} u_{+}^{2} e^{-\Phi}\right)^{1 / 2}} \text { and } u_{2}=\frac{u_{-}}{\left(\int_{D} u_{-}^{2} e^{-\Phi}\right)^{1 / 2}} \text {. }
$$

We first prove that either $u_{1}$ or $u_{2}$ is a solution of (5.1). It is obvious if $u=u_{+}$or $u=u_{-}$. Otherwise, we have $u_{+} \neq 0$ and $u_{-} \neq 0$, and the claim follows from the estimate

$$
\inf \left(\frac{\int_{D}\left|\nabla u_{+}\right|^{2} e^{-\Phi} d x}{\int_{D} u_{+}^{2} e^{-\Phi} d x}, \frac{\int_{D}\left|\nabla u_{-}\right|^{2} e^{-\Phi} d x}{\int_{D} u_{-}^{2} e^{-\Phi} d x}\right) \leq \frac{\int_{D}\left(\left|\nabla u_{+}\right|^{2}+\left|\nabla u_{-}\right|^{2}\right) e^{-\Phi} d x}{\int_{D}\left(u_{+}^{2}+u_{-}^{2}\right) e^{-\Phi} d x}=\frac{\int_{D}|\nabla u|^{2} e^{-\Phi} d x}{\int_{D} u^{2} e^{-\Phi} d x} .
$$

Up to changing $u$ into $-u$, we assume that $u_{1}$ is a solution of (5.1). Now, suppose by contradiction that $\left|\Omega_{u}\right|<m$. Then, for every ball $B_{r}\left(x_{0}\right) \subset D$ such that $\left|\Omega_{u}\right|+\left|B_{r}\right| \leq m$, writing that

$$
\int_{D}\left|\nabla u_{1}\right|^{2} e^{-\Phi} d x \leq \int_{D}\left|\nabla\left(u_{1}+t \varphi\right)\right|^{2} e^{-\Phi} d x
$$

for all functions $\varphi \in H_{0}^{1}\left(B_{r}\left(x_{0}\right)\right)$ and all $t \in \mathbb{R}$, we easily get that $u_{1}$ is a solution of

$$
-\operatorname{div}\left(e^{-\Phi} \nabla u_{1}\right)=\lambda_{m} e^{-\Phi} u_{1} \quad \text { in } \quad B_{r}\left(x_{0}\right) .
$$

By the strong maximum principle, we get $u>0$ in $B_{r}\left(x_{0}\right)$, which is a contradiction. This proves both the saturation of the constraint and the positivity of $u$.

5.3. A free-boundary problem with measure constraint. We now follow the strategy adopted in [6, 7]. In particular, the proof of Theorem 5.16 below is very close to the one of Theorem 1.5 in [6]. Note that the approach is local and that a result analogous to Theorem 5.16 with perturbations in $D$ is vain (see Remark 1.6 in [6]).

Let $u \in H_{0}^{1}(D)$ be a solution of (5.1). For any $v \in H_{0}^{1}(D)$ we set

$$
J(v):=\int_{D}|\nabla v|^{2} e^{-\Phi} d x-\lambda_{m} \int_{D} v^{2} e^{-\Phi} d x,
$$

where it is recalled that $\lambda_{m}=\int_{D}|\nabla u|^{2} e^{-\Phi} d x$.

Remark 5.9 (Removal of the integral constraint). It is plain to see that, when $u \in H_{0}^{1}(D)$ is a solution of (5.1),

$$
J(u)=\min \left\{J(v): v \in H_{0}^{1}(D),\left|\Omega_{v}\right| \leq m\right\} .
$$

For a ball $B_{r}\left(x_{0}\right) \subset \mathbb{R}^{d}$ we define the admissible set

$$
\mathcal{A}\left(u, x_{0}, r\right):=\left\{v \in H_{0}^{1}(D): u-v \in H_{0}^{1}\left(B_{r}\left(x_{0}\right)\right)\right\} .
$$

Remark 5.10 (Coercivity of $J$ ). We notice that the set $\left\{v \in \mathcal{A}\left(u, x_{0}, r\right): J(v)<C\right\}$ is weakly compact in $H_{0}^{1}(D)$. Precisely, if $u \in H_{0}^{1}(D), \Phi \in W^{1, \infty}(D)$ and $J$ be given by (5.10), then there is a constant $r_{0}>0$, depending on $d, \Phi, \lambda_{m}$ and $D$ such that for all $r \leq r_{0}$,

$$
\int_{B_{r}\left(x_{0}\right)}|\nabla v|^{2} d x \leq 2 e^{\max \Phi} J(v)+\left(1+4 \lambda_{m} e^{\max \Phi-\min \Phi}\right)\|u\|_{H^{1}(D)}^{2}, \quad \forall v \in \mathcal{A}\left(u, x_{0}, r\right) .
$$


Indeed, let $v \in \mathcal{A}\left(u, x_{0}, r\right)$ with $r \leq r_{0}$. We have

$$
\begin{aligned}
\int_{D} v^{2} d x & \leq 2 \int_{D}(v-u)^{2} d x+2 \int_{D} u^{2} d x \leq \frac{2}{\lambda_{1}\left(B_{r}\left(x_{0}\right)\right)} \int|\nabla(v-u)|^{2} d x+2 \int_{D} u^{2} d x \\
& \leq \frac{4 r_{0}^{2}}{\lambda_{1}\left(B_{1}\right)} \int_{D}\left(|\nabla v|^{2}+|\nabla u|^{2}\right) d x+2 \int_{D} u^{2} d x,
\end{aligned}
$$

where the last inequality is due to the (-2)-homogeneity of $\lambda_{1}\left(B_{r}\right)$ and the fact that $r \leq r_{0}$. Choosing $r_{0}$ small enough (depending only on $d, \lambda_{m},\|\nabla \Phi\|_{L^{\infty}}$ and the diameter of $D$ ) we get

$$
\begin{aligned}
\int_{B_{r}\left(x_{0}\right)}|\nabla v|^{2} d x & \leq e^{\max \Phi} J(v)+\lambda_{m} e^{\max \Phi-\min \Phi} \int_{D} v^{2} d x \\
& \leq e^{\max \Phi} J(v)+\frac{1}{2} \int_{B_{r}\left(x_{0}\right)}\left(|\nabla v|^{2}+|\nabla u|^{2}\right) d x+2 \lambda_{m} e^{\max \Phi-\min \Phi} \int_{D} u^{2} d x
\end{aligned}
$$

which concludes the proof of (5.12).

As a consequence, we obtain the following result, which gives us the existence of a solution to a local version of the minimization problem (5.11) with some different measure constraint.

Lemma 5.11 (Existence of local minimizers). Let $u \in H_{0}^{1}(D)$ be a solution of the problem (5.11). Let $B_{r}\left(x_{0}\right) \subset \mathbb{R}^{d}$ be a fixed ball and let $\widetilde{m}$ be a real constant such that $\widetilde{m}>\left|\Omega_{u} \backslash B_{r}\left(x_{0}\right)\right|$. Then:

(1) the problem

$$
\min \left\{J(v): v \in \mathcal{A}\left(u, x_{0}, r\right),\left|\Omega_{v}\right| \leq \widetilde{m}\right\}
$$

has a solution provided that $r \leq r_{0}$ with $r_{0}$ given by Remark 5.10 ,

(2) if $D_{r}\left(x_{0}\right):=B_{r}\left(x_{0}\right) \cap D$ is connected and $\left|\Omega_{u} \cup D_{r}\left(x_{0}\right)\right|>\tilde{m}$, then $\left|\Omega_{v}\right|=\widetilde{m}$;

(3) there exists $r_{0}>0$ such that, for every $r<r_{0}$, every solution $v$ of (5.13) is non-negative.

Proof. For 1, it is enough to notice that, by Remark 5.10, $J$ is bounded from below in $\mathcal{A}\left(u, x_{0}, r\right)$. Then, if $\left(v_{n}\right)_{n \geq 1}$ is a minimizing sequence for (5.13), by (5.12) $v_{n}$ is bounded in $H^{1}$ For 2, if $D_{r}\left(x_{0}\right)$ is connected and $\left|\Omega_{u} \cup D_{r}\left(x_{0}\right)\right|>\tilde{m}$, we argue as in the proof of Lemma 5.8 to conclude that $\left|\Omega_{v}\right|=\tilde{m}$. For 3, let $v$ be a solution of (5.13). Then, by the optimality of $v$ and the fact that $v^{+} \in \mathcal{A}\left(u, x_{0}, r\right)$ and $\Omega_{v^{+}} \subset \Omega_{v}$, one has

$$
J\left(v^{+}\right)+J\left(v^{-}\right)=J(v) \leq J\left(v^{+}\right),
$$

which means that $J\left(v^{-}\right) \leq 0$. Therefore,

$$
\begin{aligned}
\int_{B_{r}\left(x_{0}\right)}\left|\nabla v^{-}\right|^{2} e^{-\Phi} d x & \leq \lambda_{m} \int_{B_{r}\left(x_{0}\right)}\left|v^{-}\right|^{2} e^{-\Phi} d x \\
& \leq \lambda_{m} e^{2 r \tau} C_{d} r^{2} \int_{B_{r}\left(x_{0}\right)}\left|\nabla v^{-}\right|^{2} e^{-\Phi} d x
\end{aligned}
$$

where the second inequality is due to the fact that $\max _{B_{r}\left(x_{0}\right)} \Phi-\min _{B_{r}\left(x_{0}\right)} \Phi \leq 2 r \tau$ and the variational characterization and the scaling of $\lambda_{1}\left(B_{r}, 0\right)=C_{d} r^{-2}$. Thus, for $r$ small enough $\left(r \leq r_{0}\right.$ with $r_{0}$ depending only on $\tau, \lambda_{m}$ and $\left.d\right), v^{-}=0$.

5.4. An internal variation optimality condition. Let $D \subset \mathbb{R}^{d}$ be a bounded open set, $u \in$ $H_{0}^{1}(D)$ and $\xi \in C_{c}^{\infty}\left(D ; \mathbb{R}^{d}\right)$. The first variation $\delta J(u)[\xi]$, of $J$ at $u$ in the direction $\xi$, is given by

$$
\delta J(u)[\xi]:=\lim _{t \rightarrow 0} \frac{J\left(u_{t}\right)-J(u)}{t}, \quad \text { where } \quad u_{t}(x):=u(x+t \xi(x)) .
$$

A straightforward computation gives that

$$
\delta J(u)[\xi]:=\int_{D}\left[2 D \xi(\nabla u) \cdot \nabla u+\left(|\nabla u|^{2}-\lambda_{m} u^{2}\right)(\nabla \Phi \cdot \xi-\operatorname{div} \xi)\right] e^{-\Phi} d x .
$$


We prove in Proposition 5.12 the existence of an Euler-Lagrange multiplier for every solution $u$ of (5.11). This, using a local internal variation of the boundary of the optimal set $\Omega_{u}$, we derive an optimal boundary condition for $u$ (see Lemma 5.30).

Proposition 5.12 (Euler-Lagrange equation). Let $u$ be a solution of (5.11). Then, there exists $\Lambda_{u}>0$ such that

$$
\delta J(u)[\xi]=\Lambda_{u} \int_{\Omega_{u}} \operatorname{div} \xi d x \quad \text { for every } \quad \xi \in C_{c}^{\infty}\left(D ; \mathbb{R}^{d}\right) .
$$

Moreover, for every $x_{0} \in \partial \Omega_{u} \cap \partial D$ and every $r>0$, we have

$$
\delta J(u)[\xi] \geq \Lambda_{u} \int_{\Omega_{u}} \operatorname{div} \xi d x,
$$

for every $\xi \in C_{c}^{\infty}\left(B_{r}\left(x_{0}\right), \mathbb{R}^{d}\right)$ such that $(I d+\xi)^{-1}\left(D_{r}\left(x_{0}\right)\right) \subset D_{r}\left(x_{0}\right)$.

Proof. Let $\xi \in C_{c}^{\infty}\left(D ; \mathbb{R}^{d}\right)$ and $u_{t}(x)=u(x+t \xi(x))$. Then we have

$$
\left|\Omega_{u_{t}}\right|=\left|\Omega_{u}\right|-t \int_{\Omega_{u}} \operatorname{div} \xi d x+o(t)
$$

Step 1. We first notice that if $B_{r}\left(x_{0}\right) \subset \mathbb{R}^{d}$ is a ball such that

$$
D_{r}\left(x_{0}\right):=B_{r}\left(x_{0}\right) \cap D \quad \text { is connected } \quad \text { and } \quad 0<\left|D_{r}\left(x_{0}\right) \cap \Omega_{u}\right|<\left|D_{r}\left(x_{0}\right)\right| \text {, }
$$

then there is a vector field $\xi_{0} \in C_{c}^{\infty}\left(D_{r}\left(x_{0}\right) ; \mathbb{R}^{d}\right)$ such that $\int_{\Omega_{u}} \operatorname{div} \xi_{0} d x=1$. Indeed, if this is not the case, then we have

$$
\int_{\Omega_{u}} \operatorname{div} \xi d x=0 \quad \text { for every } \quad \xi \in C_{c}^{\infty}\left(D_{r}\left(x_{0}\right) ; \mathbb{R}^{d}\right) .
$$

For every ball $B_{\rho}\left(x_{1}\right) \subset D_{r}\left(x_{0}\right)$, take a vector field of the form $\xi(x)=\left(x-x_{1}\right) \phi_{\varepsilon}(x)$ with $0 \leq \phi_{\varepsilon} \leq 1$ on $B_{\rho}\left(x_{1}\right), \phi$ radially decreasing in $B_{\rho}\left(x_{1}\right)$ with $\left|\nabla \phi_{\varepsilon}\right| \leq C(\rho \varepsilon)^{-1}, \phi_{\varepsilon}=1$ on $B_{\rho(1-\varepsilon)}\left(x_{1}\right)$ and $\phi_{\varepsilon}=0$ on $\partial B_{\rho}\left(x_{1}\right)$. Then we have $\int_{\Omega_{u}}\left(d \phi_{\varepsilon}(x)+\left(x-x_{1}\right) \cdot \nabla \phi_{\varepsilon}(x)\right) d x=0$ and, passing to the limit as $\varepsilon \rightarrow 0$, we get

$$
d\left|\Omega_{u} \cap B_{\rho}\left(x_{1}\right)\right|-\rho \mathcal{H}^{d-1}\left(\Omega_{u} \cap \partial B_{\rho}\left(x_{1}\right)\right)=0 .
$$

In particular, we get that the map $\rho \mapsto \rho^{-d}\left|\Omega_{u} \cap B_{\rho}\left(x_{1}\right)\right|$ is constant. Since the above identity holds for all balls $B_{\rho}\left(x_{1}\right) \subset D_{r}\left(x_{0}\right)$, we get that $\left|\Omega_{u} \cap D_{r}\left(x_{0}\right)\right|=0$ or $\left|\Omega_{u} \cap D_{r}\left(x_{0}\right)\right|=\left|D_{r}\left(x_{0}\right)\right|$, which concludes the proof of the claim.

Step 2. We now prove the first statement of the proposition. Let $\xi_{0} \in C_{c}^{\infty}\left(D ; \mathbb{R}^{d}\right)$ be as in Step 1 and $\xi \in C_{c}^{\infty}\left(D ; \mathbb{R}^{d}\right)$. There are two cases:

If $\int_{\Omega_{u}} \operatorname{div} \xi d x=0$, define $\xi_{1}=\xi+\eta \xi_{0}$ with $\eta>0$ so that $\int_{\Omega_{u}} \operatorname{div} \xi_{1} d x=\eta$.

Set $u_{t}(x)=u\left(x+t \xi_{1}(x)\right)$. Then, for $t$ small enough, $u_{t} \in H_{0}^{1}(D),\left|\Omega_{u_{t}}\right| \leq\left|\Omega_{u}\right|=m$ and

$$
J\left(u_{t}\right)=J(u)+t \delta J(u)\left[\xi_{1}\right]+o(t) .
$$

By the minimality of $u$ we have $J(u) \leq J\left(u_{t}\right)$ and so, $\delta J(u)\left[\xi_{1}\right] \geq 0$. Therefore,

$$
\delta J(u)[\xi] \geq-\eta \delta J(u)\left[\xi_{0}\right] \text { for every } \eta>0,
$$

and hence, we get $\delta J(u)[\xi] \geq 0$. Taking $-\xi$ instead of $\xi$ we have that $\delta J(u)[\xi]=0$, and hence (5.15) holds for any $\Lambda_{u} \geq 0$.

If $\int_{\Omega_{u}} \operatorname{div} \xi d x \neq 0$, define $\xi_{2}:=\xi-\xi_{0} \int_{\Omega_{u}} \operatorname{div} \xi d x$. Then $\int_{\Omega_{u}} \operatorname{div} \xi_{2} d x=0$ and, by the preceding case, we have $\delta J(u)\left[\xi_{2}\right]=0$. On the other hand,

$$
\delta J(u)\left[\xi_{2}\right]=\delta J(u)[\xi]-\delta J(u)\left[\xi_{0}\right] \int_{\Omega_{u}} \operatorname{div} \xi d x
$$


which proves (5.15) with $\Lambda_{u}:=\delta J(u)\left[\xi_{0}\right]$. Moreover, for $t$ small enough, $u_{t}(x)=u(x+t \xi(x)) \in$ $H_{0}^{1}(D)$ and, by the minimality of $u$, we have

$$
J(u) \leq J\left(u_{t}\right)=J(u)+t \Lambda_{u}+o(t),
$$

which proves that $\Lambda_{u} \geq 0$. The strict inequality follows by a general result (Proposition A.1) for minimizers of $J$ with respect to internal perturbations.

Step 3. Let $x_{0} \in \partial \Omega_{u} \cap \partial D, r>0$ and $\xi_{0} \in C_{c}^{\infty}\left(D ; \mathbb{R}^{d}\right)$ be as in Step 1 so that we have $\delta J(u)\left[\xi_{0}\right]=\Lambda_{u}$. For any $\xi \in C_{c}^{\infty}\left(B_{r}\left(x_{0}\right), \mathbb{R}^{d}\right)$ such that $(I d+\xi)^{-1}\left(D_{r}\left(x_{0}\right)\right) \subset D_{r}\left(x_{0}\right)$, we set $\xi_{1}=\xi-(1-\eta) \xi_{0} \int_{\Omega_{u}} \operatorname{div} \xi d x$ where $\eta$ is some positive constant. Note that the vector field $\xi_{1}$ is such that $u_{t}(x)=u\left(x+t \xi_{1}(x)\right) \in H_{0}^{1}(D)$ for small $t>0$ and $\int_{\Omega_{u}} \operatorname{div} \xi_{1} d x=\eta>0$. Therefore, using the minimality of $u$, we have for every $t>0$ small enough

$$
J(u) \leq J\left(u_{t}\right)=J(u)+t \delta J(u)\left[\xi_{1}\right]+o(t),
$$

so that we get $\delta J(u)\left[\xi_{1}\right] \geq 0$. It follows that $\delta J(u)[\xi] \geq(1-\eta) \Lambda_{u}$ for every $\eta>0$, which concludes the proof.

In the following lemma we show that the Lagrange multipliers, associated to the solutions of variational problems with measure constraint in a fixed ball $B_{r}\left(x_{0}\right)$, are continuous with respect to variations of the measure constraint around $m$. This lemma will be used several times in the proof of the optimality of the blow-up limits.

Lemma 5.13 (Convergence of the Lagrange multipliers). Let $D \subset \mathbb{R}^{d}$ be a bounded open set, $u \in H_{0}^{1}(D)$ be a solution of (5.11) and $\Lambda_{u}$ be the constant from (5.15). Let $B_{r}\left(x_{0}\right) \subset \mathbb{R}^{d}$ be a ball such that

$$
D_{r}\left(x_{0}\right):=B_{r}\left(x_{0}\right) \cap D \quad \text { is connected and } \quad 0<\left|D_{r}\left(x_{0}\right) \cap \Omega_{u}\right|<\left|D_{r}\left(x_{0}\right)\right| .
$$

Let the sequence $\left(m_{n}\right)_{n \geq 1}$ be such that $\lim _{n \rightarrow \infty} m_{n}=m$. Then, for $n$ big enough, there is a solution $u_{n} \in \mathcal{A}\left(u, x_{0}, r\right)$ of the problem

$$
\min \left\{J(v): v \in \mathcal{A}\left(u, x_{0}, r\right),\left|\Omega_{v}\right| \leq m_{n}\right\} .
$$

Moreover, up to a subsequence, we have:

(a) for every $n$ there is a Lagrange multiplier $\Lambda_{u_{n}}>0$ for which (5.15) holds for $u_{n}$ in $D_{r}\left(x_{0}\right)$;

(b) for every $n$ there is a vector field $\xi_{n} \in C_{c}^{\infty}\left(D_{r}\left(x_{0}\right) ; \mathbb{R}^{d}\right)$ such that

$$
\left.\frac{d}{d t}\right|_{t=0} J\left(u_{n}^{t}\right)=\Lambda_{u_{n}} \quad \text { and }\left.\quad \frac{d}{d t}\right|_{t=0}\left|\Omega_{u_{n}^{t}}\right|=-1 \quad \text { where } \quad u_{n}^{t}(x):=u_{n}\left(x+t \xi_{n}(x)\right) \text {; }
$$

(c) $u_{n}$ converges strongly in $H_{0}^{1}(D)$ and pointwise almost everywhere to a function $u_{\infty} \in \mathcal{A}\left(u, x_{0}, r\right)$ which is a solution of (5.13);

(d) the sequence of characteristic functions $\mathbb{1}_{\Omega_{u_{n}}}$ converges to $\mathbb{1}_{\Omega_{u_{\infty}}}$ pointwise almost everywhere and strongly in $L^{2}(D)$;

(e) if we have $0<\left|\Omega_{u} \backslash B_{r}\left(x_{0}\right)\right|<\left|D \backslash B_{r}\left(x_{0}\right)\right|$, then $\lim _{n \rightarrow \infty} \Lambda_{u_{n}}=\Lambda_{u}$.

Furthermore, if $D$ is of class $C^{1,1}$ and $m_{n}<m$ for every $n$ large enough, then all these properties still hold even if the assumption $\left|\Omega_{u} \cap D_{r}\left(x_{0}\right)\right|<\left|D_{r}\left(x_{0}\right)\right|$ is not satisfied.

Proof. First of all, we notice that since $\left|\Omega_{u} \backslash D_{r}\left(x_{0}\right)\right|<m<\left|\Omega_{u} \cup D_{r}\left(x_{0}\right)\right|$, we may assume that the same holds for every $m_{n}$, for $n$ large enough. Thus, by Lemma 5.11, the problem (5.17) has a solution $u_{n}$ such that $\left|\Omega_{u_{n}}\right|=m_{n}$. Then, it follows that $u_{n}$ satisfies

$$
0<\left|\Omega_{u_{n}} \cap D_{r}\left(x_{0}\right)\right|<\left|D_{r}\left(x_{0}\right)\right| .
$$

Therefore, by step 1 in the proof of Proposition 5.12 , there exists a vector field $\xi_{n} \in C_{c}^{\infty}\left(D_{r}\left(x_{0}\right) ; \mathbb{R}^{d}\right)$ such that $\int_{\Omega_{u_{n}}} \operatorname{div} \xi_{n} d x=1$, and, reasoning as in Proposition 5.12, there exists $\Lambda_{u_{n}}>0$ such that

$$
\delta J\left(u_{n}\right)[\xi]=\Lambda_{u_{n}} \int_{\Omega_{u_{n}}} \operatorname{div} \xi d x \quad \text { for every } \quad \xi \in C_{0}^{\infty}\left(D_{r}\left(x_{0}\right), \mathbb{R}^{d}\right) .
$$


Moreover, taking $u_{n}^{t}(x)=u_{n}\left(x+t \xi_{n}(x)\right)$, we obtain (5.18). This proves (a) and (b). We notice that the only difference with Proposition 5.12 is that in the present case, $u_{n}$ is only a solution of a variational problem in $B_{r}\left(x_{0}\right)$.

Let now $n$ be fixed and $\xi_{0} \in C_{c}^{\infty}\left(B_{r}\left(x_{0}\right) ; \mathbb{R}^{d}\right)$ be the vector field, from the proof of Proposition 5.12, associated to $u$. Then, taking $u_{t}(x):=u\left(x+t \xi_{0}(x)\right)$, we have that

$$
\left.\frac{d}{d t}\right|_{t=0}\left|\Omega_{u_{t}}\right|=-\int_{\Omega_{u}} \operatorname{div} \xi_{0} d x=-1
$$

and so, for $n$ large enough, there is a unique $t_{n} \in \mathbb{R}$ such that $\left|\Omega_{u_{n}}\right|=m_{n}=\left|\Omega_{u_{t_{n}}}\right|$. In particular, there are constants $C$ and $n_{0}$, depending on $u$ and $\xi_{0}$, but not on $n$, such that

$$
J\left(u_{n}\right) \leq J\left(u_{t_{n}}\right) \leq C \text { for every } n \geq n_{0} .
$$

Then, by Remark 5.10, $\left(u_{n}\right)_{n>1}$ is uniformly bounded in $H_{0}^{1}(D)$, so up to a subsequence, $u_{n}$ converges weakly in $H^{1}$, strongly in $L^{2}$ and pointwise a.e. to a function $u_{\infty} \in \mathcal{A}\left(u, x_{0}, r\right)$. Now, since the pointwise convergence implies $\mathbb{1}_{\Omega_{u_{\infty}}} \leq \liminf \mathbb{1}_{\Omega_{u_{n}}}$, we get that $\left|\Omega_{u_{\infty}}\right| \leq \liminf m_{n}=m$. In particular, $J(u) \leq J\left(u_{\infty}\right)$. On the other hand, the weak $H^{1}$ convergence of $u_{n}$ gives that

$$
J\left(u_{\infty}\right) \leq \liminf _{n \rightarrow \infty} J\left(u_{n}\right) \leq \liminf _{n \rightarrow \infty} J\left(u_{t_{n}}\right)=J(u),
$$

so, we get $J\left(u_{\infty}\right)=J(u), u_{\infty}$ is a solution of (5.13), $\left|\Omega_{u_{\infty}}\right|=m$ (by the saturation of the constraint). Moreover, $J\left(u_{n}\right) \rightarrow J\left(u_{\infty}\right)$ since we have

$$
\limsup _{n \rightarrow \infty} J\left(u_{n}\right) \leq \limsup _{n \rightarrow \infty} J\left(u_{t_{n}}\right)=J(u) \leq J\left(u_{\infty}\right) \leq \liminf _{n \rightarrow \infty} J\left(u_{n}\right) .
$$

But $u_{n}$ strongly converges in $L^{2}(D)$ to $u_{\infty}$ so that it gives $\int_{D} e^{-\Phi}\left|\nabla u_{n}\right|^{2} d x \rightarrow \int_{D} e^{-\Phi}\left|\nabla u_{\infty}\right|^{2} d x$, which means that the convergence of $u_{n}$ to $u$ is strong in $H_{0}^{1}(D)$.

We now check that the convergence of $\mathbb{1}_{\Omega_{u_{n}}}$ to $\mathbb{1}_{\Omega_{u_{\infty}}}$ is strong in $L^{2}$. Indeed, for all non-negative function $\varphi \in L^{2}(D)$, the Fatou lemma shows that

$$
\int_{D} \mathbb{1}_{\Omega_{u_{\infty}}} \varphi \leq \int_{D} \underline{\lim } \mathbb{1}_{\Omega_{u_{n}}} \varphi \leq \underline{\lim } \int_{D} \mathbb{1}_{\Omega_{u_{n}}} \varphi
$$

Up to a subsequence, there exists $h \in L^{2}(D)$ such that $\mathbb{1}_{\Omega_{u_{n}}} \rightarrow h$ weakly in $L^{2}(D)$. Thus, (5.21) yields $\mathbb{1}_{\Omega_{u_{\infty}}} \leq h$. Moreover, $\|h\|_{2} \leq \underline{\lim }\left\|\mathbb{1}_{\Omega_{u_{\infty}}}\right\|_{2}$. As a consequence, $\|h\|_{2}=m^{1 / 2}$, which entails that $\mathbb{1}_{\Omega_{u_{n}}} \rightarrow h$ strongly in $L^{2}(D)$. Since $\mathbb{1}_{\Omega_{u_{\infty}}} \leq h$, we conclude that $\mathbb{1}_{\Omega_{u_{n}}} \rightarrow \mathbb{1}_{\Omega_{u_{\infty}}}$ strongly in $L^{2}(D)$, and so, up to a subsequence $\mathbb{1}_{\Omega_{u_{n}}}$ converges to $\mathbb{1}_{\Omega_{u_{\infty}}}$ pointwise almost everywhere. This proves (c) and (d).

In order to prove (e), we first notice that $u$ and $u_{\infty}$ are both solutions of (5.11) since $J\left(u_{\infty}\right)=$ $J(u)$. Therefore, there is a Lagrange multiplier $\Lambda_{\infty}$ such that

$$
\delta J\left(u_{\infty}\right)[\xi]=\Lambda_{\infty} \int_{\Omega_{u_{\infty}}} \operatorname{div} \xi d x \quad \text { for every } \quad \xi \in C_{c}^{\infty}\left(D ; \mathbb{R}^{d}\right),
$$

Moreover, by (c) and (d), we get that

$$
\delta J\left(u_{\infty}\right)[\xi]=\lim _{n \rightarrow \infty} \delta J\left(u_{n}\right)[\xi] \quad \text { and } \quad \int_{\Omega_{u_{\infty}}} \operatorname{div} \xi d x=\lim _{n \rightarrow \infty} \int_{\Omega_{u_{n}}} \operatorname{div} \xi d x,
$$

for every $\xi \in C_{c}^{\infty}\left(D_{r}\left(x_{0}\right) ; \mathbb{R}^{d}\right)$. Now, choosing $\xi \in C_{c}^{\infty}\left(D_{r}\left(x_{0}\right) ; \mathbb{R}^{d}\right)$ such that $\int_{\Omega_{u_{\infty}}} \operatorname{div} \xi d x \neq 0$ and using (5.22) and (5.20) we get that $\Lambda_{u_{n}}$ converges to $\Lambda_{\infty}$. Finally, if we have $0<\left|\Omega_{u} \backslash B_{r}\left(x_{0}\right)\right|<$ $\left|D \backslash B_{r}\left(x_{0}\right)\right|$, there exists $\xi \in C_{c}^{\infty}\left(D \backslash B_{r}\left(x_{0}\right) ; \mathbb{R}^{d}\right)$ such that $\int_{\Omega_{u_{\infty}}} \operatorname{div} \xi d x \neq 0$, so that $\Lambda_{\infty}=\Lambda_{u}$ since $u=u_{\infty}$ outside the ball $B_{r}\left(x_{0}\right)$.

The proof of the last statement of the Proposition is very similar. We have $\left|\Omega_{u} \backslash D_{r}\left(x_{0}\right)\right|<m=$ $\left|\Omega_{u} \cup D_{r}\left(x_{0}\right)\right|$ so that, since $m_{n}<m$, we have $\left|\Omega_{u} \backslash D_{r}\left(x_{0}\right)\right|<m_{n}<\left|\Omega_{u} \cup D_{r}\left(x_{0}\right)\right|$ for every $n$ large enough. It follows from Lemma 5.11 that the problem (5.17) has a solution $u_{n}$ with $\left|\Omega_{u_{n}}\right|=m_{n}$ and such that (5.19) holds. Note also that there exists a vector field $\xi_{0} \in C_{0}^{\infty}\left(B_{r}\left(x_{0}\right), \mathbb{R}^{d}\right)$ such 
that $\left(I d+t \xi_{0}\right)^{-1}\left(D_{r}\left(x_{0}\right)\right) \subset D_{r}\left(x_{0}\right)$ for every small $t>0$ and $\int_{\Omega_{u}} \operatorname{div} \xi_{0} d x=1$ (consider a smooth extension of the normal to the boundary of $D$ on $\partial D \cap B_{r / 2}\left(x_{0}\right)$ ). Moreover, we have $t_{n}>0$ (since $\left.m_{n}<m\right)$ and hence $u_{t_{n}} \in H_{0}^{1}(D)$. The rest of the proof is unchanged.

5.5. Almost optimality of $u$ at small scales. Let $u$ be a solution of (5.1) in $D \subset \mathbb{R}^{d}$. For $x_{0} \in \mathbb{R}^{d}$ and $h>0$, we define the upper and the lower Lagrange multipliers, $\mu_{-}\left(h, x_{0}, r\right) \geq 0$ and $\mu_{+}\left(h, x_{0}, r\right) \geq 0$, by

$\mu_{+}\left(h, x_{0}, r\right)=\inf \left\{\mu \geq 0: J(u)+\mu\left|\Omega_{u}\right| \leq J(v)+\mu\left|\Omega_{v}\right|, \forall v \in \mathcal{A}\left(u, x_{0}, r\right), m \leq\left|\Omega_{v}\right| \leq m+h\right\}$,

$\mu_{-}\left(h, x_{0}, r\right)=\sup \left\{\mu \geq 0: J(u)+\mu\left|\Omega_{u}\right| \leq J(v)+\mu\left|\Omega_{v}\right|, \forall v \in \mathcal{A}\left(u, x_{0}, r\right), m-h \leq\left|\Omega_{v}\right| \leq m\right\}$.

Remark 5.14 $\left(\mu_{-} \leq \Lambda_{u} \leq \mu_{+}\right)$. We notice that if $B_{r}\left(x_{0}\right) \subset \mathbb{R}^{d}$ is a ball such that $D_{r}\left(x_{0}\right):=$ $D \cap B_{r}\left(x_{0}\right)$ is connected and $0<\left|D_{r}\left(x_{0}\right) \cap \Omega_{u}\right|<\left|D_{r}\left(x_{0}\right)\right|$, then

$$
\mu_{-}\left(h, x_{0}, r\right) \leq \Lambda_{u} \leq \mu_{+}\left(h, x_{0}, r\right) \quad \text { for every } \quad h>0 .
$$

Indeed, by Step 1 of the proof of Proposition 5.12, there is a vector field $\xi \in C_{c}^{\infty}\left(D_{r}\left(x_{0}\right) ; \mathbb{R}^{d}\right)$ such that $\int_{\Omega_{u}} \operatorname{div} \xi d x=1$. Let $u_{t}(x)=u(x+t \xi(x))$. Then for $|t|$ small enough $u_{t} \in \mathcal{A}\left(u, x_{0}, r\right)$ and $m-h<\left|\Omega_{u_{t}}\right|<m+h$. Moreover, for every $\mu \geq 0$ we have

$$
J\left(u_{t}\right)+\mu\left|\Omega_{u_{t}}\right|=J(u)+t \Lambda_{u}+\mu\left(\left|\Omega_{u}\right|-t\right)+o(t) .
$$

Now, if $t>0$ is small enough and $\Lambda_{u}<\mu$, then $m>\left|\Omega_{u_{t}}\right|$ and, by (5.23), $J\left(u_{t}\right)+\mu\left|\Omega_{u_{t}}\right|<$ $J(u)+\mu\left|\Omega_{u}\right|$, which proves that $\Lambda_{u} \geq \mu_{-}\left(h, x_{0}, r\right)$. Analogously, if $t<0$ and $\Lambda_{u}>\mu$, then $m<\left|\Omega_{u_{t}}\right|$ and again $J\left(u_{t}\right)+\mu\left|\Omega_{u_{t}}\right|<J(u)+\mu\left|\Omega_{u}\right|$, which gives that $\Lambda_{u} \leq \mu_{+}\left(h, x_{0}, r\right)$.

Remark 5.15 (Monotonicity of $\mu_{+}$and $\mu_{-}$). We notice that the following inclusion holds:

$$
\mathcal{A}(u, x, r) \subseteq \mathcal{A}\left(u, x_{0}, r_{0}\right) \quad \text { for every } \quad B_{r}(x) \subset B_{r_{0}}\left(x_{0}\right) .
$$

In particular, for every $0<h \leq h_{0}$ and every $B_{r}(x) \subset B_{r_{0}}\left(x_{0}\right)$, we have

$$
\mu_{-}\left(h_{0}, x_{0}, r_{0}\right) \leq \mu_{-}(h, x, r) \quad \text { and } \quad \mu_{+}(h, x, r) \leq \mu_{+}\left(h_{0}, x_{0}, r_{0}\right) .
$$

Theorem 5.16 (Convergence of the upper and the lower Lagrange multipliers). Let $u$ be $a$ solution of (5.1) in the bounded open set $D \subset \mathbb{R}^{d}$ and let $\Lambda_{u}$ be given by Proposition 5.12. Then there exists a constant $r_{0}>0$, which depends only on $\tau, \lambda_{m}$ and $d$, with the following property: for every ball $B_{r}\left(x_{0}\right) \subset \mathbb{R}^{d}$ centred at $x_{0} \in \partial \Omega_{u}$ with $r \leq r_{0}$ and such that

$$
D_{r}\left(x_{0}\right):=B_{r}\left(x_{0}\right) \cap D \text { is connected and } \quad 0<\left|\Omega_{u} \cap D_{r}\left(x_{0}\right)\right|<\left|D_{r}\left(x_{0}\right)\right| \text {, }
$$

we have

$$
\lim _{h \rightarrow 0} \mu_{+}\left(h, x_{0}, r_{0}\right)=\lim _{h \rightarrow 0} \mu_{-}\left(h, x_{0}, r_{0}\right)=\Lambda_{u} .
$$

If, moreover, $D$ is of class $C^{1,1}$, then there exists a constant $r_{1}>0$, which depends only on $\tau, \lambda_{m}, d$ and $D$, such that, for every ball $B_{r}\left(x_{0}\right)$ centred at $x_{0} \in \partial \Omega_{u} \cap \partial D$ with $r \leq r_{1}$, we have

$$
\lim _{h \rightarrow 0} \mu_{-}\left(h, x_{0}, r_{0}\right)=\Lambda_{u} .
$$

Proof of Theorem 5.16: Let $x_{0} \in \partial \Omega_{u}$ be such that (5.24) holds and let $h>0$ be small. We set for simplicity $r=r_{0}, B_{r}\left(x_{0}\right)=B_{r}, \mu_{+}(h):=\mu_{+}\left(h, x_{0}, r\right)$ and $\mu_{-}(h):=\mu_{-}\left(h, x_{0}, r\right)$. We proceed in three steps.

Step 1. We first prove that $\mu_{+}(h)$ is finite. Let, for any $n \in \mathbb{N}, v_{n} \in \mathcal{A}\left(u, x_{0}, r\right)$ be a solution of the variational problem

$$
\min \left\{J(v)+n\left(\left|\Omega_{v}\right|-m\right)_{+}: v \in \mathcal{A}\left(u, x_{0}, r\right),\left|\Omega_{v}\right| \leq m+h\right\} .
$$

If there exists $n$ such that $\left|\Omega_{v_{n}}\right| \leq m$, then $\mu_{+}(h) \leq n$ and hence $\mu_{+}(h)$ is finite. Indeed, by the minimality of $u$ and the definition of $v_{n}$, we have for every $v \in \mathcal{A}\left(u, x_{0}, r\right)$ such that $m \leq\left|\Omega_{v}\right| \leq m+h$

$$
J(u)+n\left|\Omega_{u}\right| \leq J\left(v_{n}\right)+n\left|\Omega_{u}\right| \leq J(v)+n\left|\Omega_{v}\right|,
$$


so that $\mu_{+}(h) \leq n$ and the inequality $\mu_{+}(h)<\infty$ holds.

Suppose, by contradiction, that $\left|\Omega_{v_{n}}\right|>m$ for every $n$. First notice that since $J\left(v_{n}\right)$ is bounded from below (see Remark 5.10) and $J\left(v_{n}\right)+n\left(\left|\Omega_{v_{n}}\right|-m\right) \leq J(u)$, we have that $\left|\Omega_{v_{n}}\right| \rightarrow m$ as $n \rightarrow \infty$. Since $v_{n}$ is a solution of (5.17) with $m_{n}:=\left|\Omega_{v_{n}}\right|$, there is a Lagrange multiplier $\Lambda_{v_{n}}$ such that (5.15) holds for $v_{n}$ and a vector field $\xi_{n}$ such that (5.18) holds for $v_{n}^{t}(x)=v_{n}\left(x+t \xi_{n}(x)\right)$. For $t>0$ small enough, $v_{n}^{t} \in \mathcal{A}\left(u, x_{0}, r\right)$ and $m<\left|\Omega_{v_{n}^{t}}\right|<m+h$. Then, by the minimality of $v_{n}$ we have

$$
J\left(v_{n}\right)+n\left(\left|\Omega_{v_{n}}\right|-m\right) \leq J\left(v_{n}^{t}\right)+n\left(\left|\Omega_{v_{n}^{t}}\right|-m\right)=J\left(v_{n}\right)+t \Lambda_{v_{n}}+n\left(\left|\Omega_{v_{n}}\right|-t-m\right)+o(t),
$$

which implies $n \leq \Lambda_{v_{n}}$, in contradiction with $\lim _{n \rightarrow \infty} \Lambda_{u_{n}}=\Lambda_{u}$ from Lemma 5.13.

Step 2. $\lim _{h \rightarrow 0} \mu_{+}(h)=\Lambda_{u}$. Let $\left(h_{n}\right)_{n \geq 1}$ be a decreasing sequence such that $h_{n} \rightarrow 0$. Since $\Lambda_{u} \leq \mu_{+}(h)$ and $h \mapsto \mu_{+}(h)$ is non-decreasing, it is sufficient to prove that $\lim _{n \rightarrow \infty} \mu_{+}\left(h_{n}\right)=\Lambda_{u}$. Fix $\varepsilon \in\left(0, \Lambda_{u}\right)$ and let $0<\alpha_{n}:=\mu_{+}\left(h_{n}\right)-\varepsilon<\mu_{+}\left(h_{n}\right)$. Let $u_{n}$ be the solution of the problem

$$
\min \left\{J(v)+\alpha_{n}\left(\left|\Omega_{v}\right|-m\right)^{+}: v \in \mathcal{A}\left(u, x_{0}, r\right),\left|\Omega_{v}\right| \leq m+h_{n}\right\} .
$$

Notice that $\left|\Omega_{u_{n}}\right|>m$, since otherwise we would have $J(u) \leq J\left(u_{n}\right)+\alpha_{n}\left(\left|\Omega_{u_{n}}\right|-m\right)^{+}$, which contradicts the definition of $\mu_{+}\left(h_{n}\right)$. For $n$ large enough, (5.24) holds with $u_{n}$, and since $u_{n}$ is solution of (5.17) with $m_{n}=\left|\Omega_{u_{n}}\right|$, by Proposition 5.12, there is a Lagrange multiplier $\Lambda_{u_{n}} \geq 0$ and a vector field $\xi_{n}$ such that (5.18) holds for $u_{n}^{t}(x):=u_{n}\left(x+t \xi_{n}(x)\right)$. By the minimality of $u_{n}$, for $t>0$ small enough, we have

$$
J\left(u_{n}\right)+\alpha_{n}\left(\left|\Omega_{u_{n}}\right|-m\right) \leq J\left(u_{n}^{t}\right)+\alpha_{n}\left(\left|\Omega_{u_{n}^{t}}\right|-m\right)=J\left(u_{n}\right)+t \Lambda_{u_{n}}+\alpha_{n}\left(\left|\Omega_{u_{n}}\right|-t-m\right)+o(t),
$$

which shows that $\Lambda_{u_{n}} \geq \alpha_{n}$. By Lemma 5.13 we have

$$
\lim _{n \rightarrow \infty} \mu_{+}\left(h_{n}\right)-\varepsilon=\lim _{n \rightarrow \infty} \alpha_{n} \leq \lim _{n \rightarrow \infty} \Lambda_{u_{n}}=\Lambda_{u},
$$

which proves the claim since $\varepsilon>0$ is arbitrary.

Step 3. $\lim _{h \rightarrow 0} \mu_{-}(h)=\Lambda_{u}$. We prove this result for any $x_{0} \in \partial \Omega_{u}$, which will conclude the proof of the Theorem. Note that the smoothness of $D$ implies that there exists a constant $c_{D}>0$ such that $D_{r}\left(x_{0}\right)$ is connected for every $r \leq r_{D}$ and every $x_{0} \in \partial \Omega_{u} \cap \partial D$.

Let $\varepsilon>0$ and $\left(h_{n}\right)_{n \in \mathbb{N}}$ be a decreasing infinitesimal sequence. We will show that $\Lambda_{u}-\varepsilon \leq$ $\lim _{n \rightarrow \infty} \mu_{-}\left(h_{n}\right)$. Let $u_{n}$ be a solution of the problem

$$
\min \left\{J(v)+\left(\mu_{-}\left(h_{n}\right)+\varepsilon\right)\left(\left|\Omega_{v}\right|-\left(m-h_{n}\right)\right)^{+}: v \in \mathcal{A}\left(u, x_{0}, r\right),\left|\Omega_{v}\right| \leq m\right\} .
$$

Up to replacing $u_{n}$ by $u_{n}^{+}$, we can assume that $u_{n} \geq 0$ in $B_{r}$ (the argument is similar to the proof of Lemma 5.11). We claim that

$$
m-h_{n} \leq\left|\Omega_{u_{n}}\right|<m .
$$

Suppose that $\left|\Omega_{u_{n}}\right|=m$. By the minimality of $u$ and $u_{n}$ we get

$$
J(u)+\left(\mu_{-}\left(h_{n}\right)+\varepsilon\right)\left|\Omega_{u}\right| \leq J\left(u_{n}\right)+\left(\mu_{-}\left(h_{n}\right)+\varepsilon\right)\left|\Omega_{u_{n}}\right| \leq J(v)+\left(\mu_{-}\left(h_{n}\right)+\varepsilon\right)\left|\Omega_{v}\right|,
$$

for every $v \in \mathcal{A}\left(u, x_{0}, r\right)$ such that $m-h_{n} \leq\left|\Omega_{v}\right| \leq m$, which contradicts the definition of $\mu_{-}\left(h_{n}\right)$. Now, if $\left|\Omega_{u_{n}}\right|<m-h_{n}$, we have $J\left(u_{n}\right) \leq J\left(u_{n}+t \varphi\right)$ for every $\varphi \in C_{c}^{\infty}\left(D_{r}\left(x_{0}\right)\right)$ with sufficiently small compact support. Thus $u_{n}$ solves the $\mathrm{PDE}-\operatorname{div}\left(e^{-\Phi} \nabla u_{n}\right)=\lambda_{m} e^{-\Phi} u_{n}$ in $D_{r}\left(x_{0}\right)$. Since $u_{n} \geq 0$ in $D_{r}\left(x_{0}\right)$, by the strong maximum principle, we have that either $u_{n} \equiv 0$ or $u_{n}>0$ in $D_{r}\left(x_{0}\right)$, in contradiction with (5.19). Thus, we proved (5.27).

We have that $u_{n}$ is solution of (5.17) with $m_{n}:=\left|\Omega_{u_{n}}\right|$ which converges to $m$ as $n \rightarrow \infty$. By Lemma 5.13, we have an Euler-Lagrange equation for $u_{n}$ in $B_{r}$ for some $\Lambda_{u_{n}}$. Let $\xi_{n} \in$ $C_{c}^{\infty}\left(D_{r}\left(x_{0}\right) ; \mathbb{R}^{d}\right)$ be the vector field from Lemma 5.13 (b) and let $u_{n}^{t}(x)=u_{n}\left(x+t \xi_{n}(x)\right)$. For negative $t<0$ and $|t|$ small enough, $u_{n}^{t} \in \mathcal{A}\left(u, x_{0}, r\right)$ and $\left|\Omega_{u_{n}}\right| \leq\left|\Omega_{u_{n}^{t}}\right|<m$. Thus, by the minimality of $u_{n}$, we get

$J\left(u_{n}\right)+\left(\mu_{-}\left(h_{n}\right)+\varepsilon\right)\left(\left|\Omega_{u_{n}}\right|-\left(m-h_{n}\right)\right) \leq J\left(u_{n}\right)+\Lambda_{u_{n}} t+\left(\mu_{-}\left(h_{n}\right)+\varepsilon\right)\left(\left|\Omega_{u_{n}}\right|-t-\left(m-h_{n}\right)\right)+o(t)$, 
which implies that $\Lambda_{u_{n}} \leq \mu_{-}\left(h_{n}\right)+\varepsilon$. Now, by Lemma 5.13, we get

$$
\Lambda_{u}=\lim _{n \rightarrow \infty} \Lambda_{u_{n}} \leq \lim _{n \rightarrow \infty} \mu_{-}\left(h_{n}\right)+\varepsilon,
$$

which conclude the proof.

Remark 5.17 (Quasi-minimality at small scales). Suppose that $D \subset \mathbb{R}^{d}$ is just a bounded open set. By the monotonicity of $\mu_{+}$and $\mu_{-}$with respect to the inclusion (Remark 5.15 ) and a covering argument we get that for every compact set $\mathcal{K} \subset D$ there is $r(\mathcal{K})>0$ such that: for every $\varepsilon>0$ there is $h>0$ such that

$$
\mu_{+}(h, x, r)-\varepsilon \leq \Lambda_{u} \leq \mu_{-}(h, x, r)+\varepsilon \quad \text { for every } \quad x \in \mathcal{K} \cap \partial \Omega_{u} \quad \text { and every } \quad 0<r \leq r(\mathcal{K}) .
$$

If, moreover, $D$ is of class $C^{1,1}$, then then exists $r_{D}>0$ such that, for every $\varepsilon>0$ there exists $h>0$ such that: for every $0<r \leq r_{D}$ and every $x_{0} \in \partial \Omega_{u}$ we have

$$
\begin{aligned}
& \mu_{+}(h, x, r)-\varepsilon \leq \Lambda_{u} \leq \mu_{-}(h, x, r)+\varepsilon \quad \text { if }\left|\Omega_{u} \cap D_{r}\left(x_{0}\right)\right|<\left|D_{r}\left(x_{0}\right)\right|, \\
& \Lambda_{u} \leq \mu_{-}(h, x, r)+\varepsilon \quad \text { otherwise. }
\end{aligned}
$$

5.6. Lipschitz continuity of the eigenfunctions on the optimal sets. In this subsection we prove that the solutions of (5.1) are (locally) Lipschitz continuous in $D$. For $\delta>0$ we set $D_{\delta}=\{x \in D: d(x, \partial D)>\delta\}$ and let $\mu>0$ be fixed. By Theorem 5.16] and Remark 5.17 we get that if $u$ is a solution of (5.1) and $\mu>\Lambda_{u}$, then there is $r_{0}>0$ such that, for every $x_{0} \in \partial \Omega_{u} \cap D_{\delta}$, we have

$$
J(u)+\mu\left|\Omega_{u}\right| \leq J(v)+\mu\left|\Omega_{v}\right| \quad \text { for every } \quad v \in \mathcal{A}\left(u, x_{0}, r_{0}\right) \text { such that }\left|\Omega_{v}\right| \geq\left|\Omega_{u}\right| .
$$

Note that the condition $\left|\Omega_{v}\right| \leq\left|\Omega_{u}\right|+h$ can be dropped by choosing $r_{0}$ such that $\left|B_{r_{0}}\right| \leq h$. We will prove that if $u \in H^{1}\left(B_{r_{0}}\right)$ is bounded, nonnegative and satisfies (5.5) and (5.28), then $u$ is Lipschitz in $D_{\delta}$.

Proposition 5.18 (Lipschitz continuity of the eigenfunctions on the optimal sets). Let $D \subset \mathbb{R}^{d}$ be a bounded open set. Let $\tau \geq 0, m \in(0,|D|)$ and $\Phi \in W^{1, \infty}(D)$. Then, every solution of (5.1) is locally Lipschitz continuous in D. More precisely, it is Lipschitz in $D_{\delta}$ for all $\delta>0$. Moreover, if the box $D$ is of class $C^{1,1}$, then $u$ (extended by 0 outside D) is Lipschitz in $\mathbb{R}^{d}$.

The proof is based on the following lemma, whose (more general) two-phase counterpart can be found for instance in [3], [5] and [10].

Lemma 5.19 (A bound on the measure div $\left(e^{-\Phi} \nabla u\right)$ ). Let $u$ be a solution of (5.11) and let $r_{0}>0$ be such that $u$ satisfies (5.28) for some $\mu>\Lambda_{u}$. Then, there is a constant $C>0$ such that for every $x_{0} \in \partial \Omega_{u} \cap D_{\delta}$ we have

$$
\left|\operatorname{div}\left(e^{-\Phi} \nabla u\right)\right|\left(B_{r}(x)\right) \leq C r^{d-1} \quad \text { for every ball } \quad B_{2 r}(x) \subset B_{r_{0}}\left(x_{0}\right) .
$$

Proof. Let $x=0$ and $\eta \in C_{c}^{\infty}\left(B_{2 r}\right)$ be such that

$$
0 \leq \eta \leq 1 \text { in } B_{2 r}, \quad \eta=1 \text { in } B_{r}, \quad\|\nabla \eta\|_{L^{\infty}} \leq \frac{C_{d}}{r} .
$$

Using $u+t \eta$ as a test function for $J$, and setting $\langle f, g\rangle:=\int_{D} f g d x$, we get

$$
2\left\langle\operatorname{div}\left(e^{-\Phi} \nabla u\right)+\lambda_{m} u e^{-\Phi}, \eta\right\rangle \leq t J(\eta)+\frac{\mu}{t}\left|B_{2 r}\right| \leq C\left(t\|\nabla \eta\|_{L^{2}}^{2}+\frac{r^{d}}{t}\right)
$$

where the constant $C>0$ depends on $d, \Phi$ and $\mu$. Now, minimizing over $t>0$ and using the estimate $\|\nabla \eta\|_{L^{2}} \leq C_{d} r^{\frac{d}{2}-1}$, we get

$$
\left\langle\operatorname{div}\left(e^{-\Phi} \nabla u\right)+\lambda_{m} u e^{-\Phi}, \eta\right\rangle \leq C r^{d-1} .
$$


By Lemma 5.5. we have that $\operatorname{div}\left(e^{-\Phi} \nabla u\right)+\lambda_{m} u e^{-\Phi}$ is a positive Radon measure. Thus, the inequality $\eta \geq \mathbb{1}_{B_{r}}$ and the boundedness of $u$ imply

$$
\left|\operatorname{div}\left(e^{-\Phi} \nabla u\right)\right|\left(B_{r}\right) \leq \lambda_{m} \int_{B_{r}} u e^{-\Phi} d x+\left\langle\operatorname{div}\left(e^{-\Phi} \nabla u\right)+\lambda_{m} u e^{-\Phi}, \mathbb{1}_{B_{r}}\right\rangle \leq C r^{d-1} .
$$

The main ingredients of the proof of Proposition 5.18 will be Lemma 5.19 and the classical gradient estimate that we recall in the lemma below.

Lemma 5.20 (Gradient estimate). Let $p>d$. Let $\mathcal{U}$ be an open subset of $\mathbb{R}^{d}$ and let $u \in$ $W_{\text {loc }}^{2, p}(\mathcal{U}) \cap L^{p}(\mathcal{U})$ be a (strong) solution to the equation

$$
\sum_{i, j=1}^{d} a_{i j}(x) \partial_{i j} u+\sum_{i=1}^{d} b_{i}(x) \partial_{i} u=f \quad \text { in } \quad \mathcal{U},
$$

where $f \in L^{p}(\mathcal{U})$ and we suppose that:

(a) the functions $a_{i j}: \mathcal{U} \rightarrow \mathbb{R}$ are Hölder continuous, that is, there are constants $C_{a}>0$ and $\delta_{a}>0$ such that

$$
\left|a_{i j}(x)-a_{i j}(y)\right| \leq C_{a}|x-y|^{\delta_{a}} \quad \text { for every } \quad x, y \in \mathcal{U} ;
$$

(b) there is a constant $M>0$ such that

$$
\left\|a_{i j}\right\|_{L^{\infty}(\mathcal{U})} \leq M \quad \text { and } \quad\left\|b_{i}\right\|_{L^{\infty}(\mathcal{U})} \leq M \quad \text { for every } \quad 1 \leq i, j \leq d ;
$$

(c) the matrix is $\left(a_{i j}\right)_{i j}$ is uniformly elliptic, that is, there is a constant $c_{a}>0$ such that

$$
\sum_{i, j=1}^{d} a_{i j}(x) \xi_{i} \xi_{j} \geq c_{a}|\xi|^{2} \quad \text { for every } \quad x \in \mathcal{U} \quad \text { and } \quad \xi=\left(\xi_{1}, \ldots, \xi_{d}\right) \in \mathbb{R}^{d} .
$$

Then, for any domain $\mathcal{U}^{\prime} \subset \subset \mathcal{U}$, we have

$$
\|u\|_{C^{1, \gamma}\left(\mathcal{U}^{\prime}\right)} \leq C\left(\|u\|_{L^{p}(\mathcal{U})}+\|f\|_{L^{p}(\mathcal{U})}\right) \quad \text { where } \quad \gamma=1-\frac{d}{p},
$$

and $C$ is a constant depending on $d, p, M, C_{a}, \delta_{a}, c_{a}, \mathcal{U}^{\prime}$ and $\mathcal{U}$.

Proof. First notice that by [27, Theorem 9.11], there is a constant $C^{\prime}$ such that

$$
\|u\|_{W^{2, p}\left(\mathcal{U}^{\prime}\right)} \leq C^{\prime}\left(\|u\|_{L^{p}(\mathcal{U})}+\|f\|_{L^{p}(\mathcal{U})}\right) .
$$

Now, the claim follows by the Sobolev inequality (see [24, Section 5.6, Theorem 6]).

Proof of Proposition 5.18, Let $u$ be a solution of (5.1). We proceed in four steps.

Step 1. $\Omega_{u}$ is open. Let $\bar{x} \in \partial \Omega_{u} \cap D$. We will prove that $u(\bar{x})=0$. Let $r_{1}>0$ be such that $B_{r_{1}}(\bar{x}) \subset D$ and let $x_{n} \in B_{r_{1} / 2}(\bar{x})$ be a sequence converging to $\bar{x}$ such that $u\left(x_{n}\right)=0$ (such a sequence exists by Lemma [5.7). By Lemma 5.19] and Lemma 5.5 (5.7), for every $n$ and every $r \leq r_{1} / 2$ we have

$$
f_{\partial B_{r}\left(x_{n}\right)} u d \mathcal{H}^{d-1} \leq u\left(x_{n}\right)+C r=C r,
$$

where the constant $C$ does not depend on $n$. Passing to the limit as $n \rightarrow \infty$, we get that

$$
f_{\partial B_{r}(\bar{x})} u d \mathcal{H}^{d-1} \leq C r \quad \text { for every } \quad r \leq r_{1} / 2
$$

which, passing to the limit as $r \rightarrow 0$, proves that $u(\bar{x})=0$.

Step 2. Gradient estimate in $\Omega_{u}$. We claim that, for every ball $B_{r}(\bar{x}) \subset \Omega_{u}$, there is a constant $C_{2}$, depending only on $\Phi, d$ and $\lambda_{m}$, such that

$$
\|\nabla u\|_{L^{\infty}\left(B_{r / 2}(\bar{x})\right)} \leq \frac{C_{2}}{r}\|u\|_{L^{\infty}\left(B_{r}(\bar{x})\right)} .
$$


Indeed, suppose that $\bar{x}=0$ and set $\Phi_{r}(x):=\Phi(r x)$ and $u_{r}(x)=u(r x)$. Then $u_{r}$ is a solution of

$$
\operatorname{div}\left(e^{-\Phi_{r}} \nabla u_{r}\right)+\lambda_{m} e^{-\Phi_{r}} u_{r}=0 \quad \text { in } \quad B_{1},
$$

which can be re-written as

$$
\Delta u_{r}-\nabla \Phi \cdot \nabla u_{r}=-\lambda_{m} u_{r} \quad \text { in } \quad B_{1} .
$$

Applying Lemma 5.20 with $\mathcal{U}=B_{1}, \mathcal{U}^{\prime}=B_{1 / 2}, a_{i j}=\delta_{i j}, b=\nabla \Phi, M=1+\|\nabla \Phi\|_{L^{\infty}}, f=-\lambda_{m} u$ and any $p>d$, we get

$$
\left\|\nabla u_{r}\right\|_{L^{\infty}\left(B_{1 / 2}\right)} \leq\left\|u_{r}\right\|_{C^{1, \alpha}\left(B_{1 / 2}\right)} \leq C_{2}\left\|u_{r}\right\|_{L^{\infty}\left(B_{1}\right)}
$$

which, after rescaling, is precisely (5.30).

Step 3. Proof of the local Lipschitz continuity. Let $\bar{x} \in \Omega_{u} \cap D_{\delta}$ and set $r:=\operatorname{dist}\left(\bar{x}, \partial \Omega_{u}\right)$. Let $r_{0} \in(0, \delta / 2)$ be such that $u$ satisfies (5.28) for every point $x_{0}$ on $\partial \Omega_{u} \cap D_{\delta / 2}$ (such an $r_{0}$ exists by a standard covering argument). We now consider two cases

Case 1. If $r \geq r_{0} / 6$, then the estimate (5.30) gives $|\nabla u(\bar{x})| \leq C_{r_{0}}$.

Case 2. If $r \leq r_{0} / 6$, let $\bar{y}$ be the projection of $\bar{x}$ on $\partial \Omega_{u}$, that is, $\bar{y} \in \partial \Omega_{u}$ and $r=|\bar{x}-\bar{y}|$. Notice that in this case we have that $\bar{y} \in \partial \Omega_{u} \cap D_{\delta / 2}$. Now, take any $\bar{z} \in B_{r}(\bar{x})$. By Lemma $[5.19$ and by the estimate (5.7) of Lemma 5.5, we have

$$
u(\bar{z}) \leq f_{\partial B_{s}(\bar{z})} u d \mathcal{H}^{d-1}+C s \quad \text { for every } \quad 0<s \leq r
$$

where $C$ is the constant in the right-hand side of (5.7). Now, multiplying by $s^{d-1}$ and then integrating from 0 to $r$ the above inequality, we get

$$
\begin{aligned}
u(\bar{z}) \leq f_{B_{r}(\bar{z})} u d x+C r & \leq 3^{d} f_{B_{3 r}(\bar{y})} u d x+C r \\
& =\frac{d}{r^{d}} \int_{0}^{3 r} s^{d-1} d s f_{\partial B_{s}(\bar{y})} u d \mathcal{H}^{d-1}+C r .
\end{aligned}
$$

Using again (5.7), this time for $\bar{y}$ (at which $u(\bar{y})=0$ by Step 1 of the proof), we get that

$$
u(\bar{z}) \leq 3^{d+1} C r \quad \text { for every } \quad \bar{z} \in B_{r}(\bar{x}) .
$$

Finally, using the estimate (5.30) this gives

$$
|\nabla u(\bar{x})| \leq\|\nabla \bar{u}\|_{L^{\infty}\left(B_{r / 2}(\bar{x})\right)} \leq \frac{C_{2}}{r}\|u\|_{L^{\infty}\left(B_{r}(\bar{x})\right)} \leq 3^{d+1} C_{2} C .
$$

This proves that $|\nabla u|$ is bounded in $D_{\delta}$ without assuming any regularity of $D$.

Step 4. Global Lipschitz estimate. We first notice that since $D$ is $C^{1,1}$ regular, the radius $r_{0}$ for which (5.28) holds does not depend on the point $x_{0} \in \partial \Omega_{u}$. Now, let $\bar{x} \in \Omega_{u} \backslash D_{r_{0}}$ and set $r:=\operatorname{dist}\left(\bar{x}, \partial \Omega_{u} \cap D\right)$. We consider the projection $\bar{y}$ of $\bar{x}$ on $\partial \Omega_{u}$ and we distinguish two cases. If $6 r \leq \operatorname{dist}(\bar{x}, \partial D)$, then we apply the estimate from Step 3 and we get that $|\nabla u(\bar{x})| \leq C$. If $6 r \geq \operatorname{dist}(\bar{x}, \partial D)$, we consider the solution $w$ to the problem

$$
-\operatorname{div}\left(e^{-\Phi} \nabla w\right)=1 \quad \text { in } \quad D, \quad w \in H_{0}^{1}(D),
$$

which is Lipschitz continuous in $\mathbb{R}^{d}$ since $D$ is of class $C^{1,1}$ (see for example [27, Theorem 9.13]). Moreover, by the strong maximum principle, we have that $u \leq C w$ for some constant $C$ depending on $\lambda_{m}, d$ and $\Phi$. Therefore, setting $r_{1}=\operatorname{dist}(\bar{x}, \partial D)$, we have for every $\bar{z} \in B_{r_{1}}(\bar{x})$,

$$
u(\bar{z}) \leq C w(\bar{z}) \leq C|\bar{z}-\bar{y}| \leq C r_{1},
$$

and we conclude by the gradient estimate (5.30). 
5.7. Non-degeneracy of the eigenfunctions and finiteness of the perimeter of $\Omega_{u}$. Let $u$ be a solution of (5.1) in the bounded open set $D \subset \mathbb{R}^{d}$. Let $x_{0} \in \partial \Omega_{u}$ and $r_{0}\left(x_{0}\right)$ be such that for every $0<r \leq r\left(x_{0}\right)$ the set $D_{r}\left(x_{0}\right):=B_{r}\left(x_{0}\right) \cap D$ is connected. Notice that such an $r\left(x_{0}\right)$ trivially exists if $x_{0} \in \partial \Omega_{u} \cap D$, while in the general case it is sufficient to assume some a priori regularity of the box $D$. Then, by Remark 5.17 , for every $\mu<\Lambda_{u}$ there is some $r_{0}>0$ such that, for every $x_{0} \in \partial \Omega_{u}$, we have

$$
J(u)+\mu\left|\Omega_{u}\right| \leq J(v)+\mu\left|\Omega_{v}\right| \quad \text { for every } \quad v \in \mathcal{A}\left(u, x_{0}, r_{0}\right) \quad \text { such that } \quad\left|\Omega_{v}\right| \leq\left|\Omega_{u}\right| .
$$

This property was first exploited by Alt and Caffarelli to prove the non-degeneracy of the solutions. More recently, it was exploited by Bucur who introduced the notion of a shape subsolution which found application to several shape optimization problems (see for example [8] and [11]).

Lemma 5.22 below is a fundamental step in the proof of the regularity of the free boundary since it allows to prove that the blow-up limits (see Subsection 5.8) are non trivial. It is the analogue of the non-degeneracy estimate from [2] and the proof is based on the same idea. Before we state it, we recall the following boundary estimate for solutions to elliptic PDEs.

Lemma 5.21 (Boundary gradient estimate). Let $p>d$. Let $\mathcal{U}$ be a bounded connected open subset of $\mathbb{R}^{d}$ with $C^{1,1}$ boundary. Let $T_{1}, \ldots, T_{k}$ be the connected components of the boundary $\partial \mathcal{U}$ and let $c_{1}, \ldots, c_{k}$ are given constants. Let $u \in W_{\text {loc }}^{2, p}(\mathcal{U}) \cap L^{p}(\mathcal{U})$ be a (strong) solution to the problem

$$
\sum_{i, j=1}^{d} a_{i j}(x) \partial_{i j} u+\sum_{i=1}^{d} b_{i}(x) \partial_{i} u=f \quad \text { in } \quad \mathcal{U}, \quad u=c_{i} \quad \text { in } \quad T_{i}, \quad i=1, \ldots, k,
$$

where $f \in L^{p}(\mathcal{U})$ and we suppose that $A=\left(a_{i j}\right)_{i j}$ and $b=\left(b_{1}, \ldots, b_{d}\right)$ satisfy the conditions (a), (b) and (c) of Lemma 5.21. Then, we have

$$
\|u\|_{C^{1, \gamma}(\mathcal{U})} \leq C\left(\|u\|_{L^{p}(\mathcal{U})}+\|f\|_{L^{p}(\mathcal{U})}\right) \quad \text { where } \quad \gamma=1-\frac{d}{p},
$$

and $C$ is a constant depending on $d, p, M, C_{a}, \delta_{a}, c_{a}$ (defined in Lemma 5.20), and $\mathcal{U}$.

Proof. By [27, Theorem 9.13], there is a constant $C^{\prime}$ such that

$$
\|u\|_{W^{2, p}(\mathcal{U})} \leq C^{\prime}\left(\|u\|_{L^{p}(\mathcal{U})}+\|f\|_{L^{p}(\mathcal{U})}\right) .
$$

The claim follows by the Sobolev inequality (see for instance [24, Section 5.6, Theorem 6]).

Lemma 5.22 (Non-degeneracy of the eigenfunctions on the optimal sets). Let $u$ be a solution of (5.1) in the bounded open set $D \subset \mathbb{R}^{d}$. Suppose that $x_{0} \in \partial \Omega_{u}, 0<\mu<\Lambda_{u}$ and $r_{0}>0$ are such that (5.32) holds. Then there are constants $c>0$ and $r_{1}>0$ which depend only on $\tau, \lambda_{m}, \mu$ and $d$, such that for every ball $B_{2 r}(x) \subset B_{r_{0}}\left(x_{0}\right)$ with $r \leq r_{1}$, we have that:

$$
\text { If }\|u\|_{L^{\infty}\left(B_{2 r}(x)\right)} \leq c r, \quad \text { then } u=0 \quad \text { in } B_{r}(x) .
$$

Proof. Let $r, x$ be such that $B_{2 r}(x) \subset B_{r_{0}}\left(x_{0}\right)$ and $\|u\|_{L^{\infty}\left(B_{2 r}(x)\right)}<c r$. Assume for simplicity that $x=0$. Let $\eta \in H^{1}\left(B_{2 r}\right)$ be the solution of the problem

$$
-\operatorname{div}\left(e^{-\Phi} \nabla \eta\right)=\beta e^{-\Phi} \quad \text { in } \quad B_{2 r} \backslash B_{r}, \quad \eta=0 \quad \text { in } \quad B_{r}, \quad \eta=c r \quad \text { in } \quad D \backslash B_{2 r},
$$

where $\beta>0$ will be chosen later. Consider the test function $\tilde{u} \in H_{0}^{1}(D)$ defined as

$$
\tilde{u}=u \wedge \eta \quad \text { in } \quad B_{2 r}, \quad \tilde{u}=u \quad \text { in } \quad D \backslash B_{2 r} .
$$

By (5.32), we get

$$
\int_{D}|\nabla u|^{2} e^{-\Phi} d x-\lambda_{m} \int_{D} u^{2} e^{-\Phi} d x+\mu\left|\Omega_{u}\right| \leq \int_{D}|\nabla \tilde{u}|^{2} e^{-\Phi} d x-\lambda_{m} \int_{D} \tilde{u}^{2} e^{-\Phi} d x+\mu\left|\Omega_{\tilde{u}}\right| .
$$


Let $E(u, r):=\int_{B_{r}}|\nabla u|^{2} e^{-\Phi} d x+\mu\left|\Omega_{u} \cap B_{r}\right|$. Since $\tilde{u} \equiv 0$ in $B_{r}$ we have $\left|\Omega_{u}\right|-\left|\Omega_{\tilde{u}}\right|=\left|\Omega_{u} \cap B_{r}\right|$ and $\int_{B_{r}} \tilde{u}^{2} d x=\int_{B_{r}}|\nabla \tilde{u}|^{2} d x=0$. Thus, we can rewrite (5.33) in the form

$$
E(u, r) \leq \int_{B_{2 r} \backslash B_{r}}\left(|\nabla \tilde{u}|^{2}-|\nabla u|^{2}\right) e^{-\Phi} d x+4 c r \lambda_{m} \int_{B_{2 r} \backslash B_{r}}(u-\tilde{u}) e^{-\Phi} d x+\lambda_{m} \int_{B_{r}} u^{2} e^{-\Phi} d x,
$$

where in the estimate of the second term we used that in $B_{r}$

$$
u^{2}-\tilde{u}^{2}=(u+\tilde{u})(u-\tilde{u}) \leq 2 u(u-\tilde{u}) \leq 2 c r(u-\tilde{u}) .
$$

Next, we estimate the first term of the right-hand side of (5.34). We have

$$
|\nabla \tilde{u}|^{2}-|\nabla u|^{2}=-|\nabla(\tilde{u}-u)|^{2}+2 \nabla \tilde{u} \cdot \nabla(\tilde{u}-u) \leq 2 \nabla \tilde{u} \cdot \nabla(\tilde{u}-u) .
$$

Integrating by parts and using that $(u-\eta)_{+}=0$ on $\partial B_{2 r}$, we get

$$
\begin{aligned}
\int_{B_{2 r} \backslash B_{r}} \nabla \tilde{u} \cdot \nabla(\tilde{u}-u) e^{-\Phi} d x & =-\int_{B_{2 r} \backslash B_{r}} \nabla \eta \cdot \nabla\left[(u-\eta)_{+}\right] e^{-\Phi} d x \\
& \leq-\beta \int_{B_{2 r} \backslash B_{r}}(u-\eta)_{+} e^{-\Phi} d x+\|\nabla \eta\|_{L^{\infty}\left(\partial B_{r}\right)} \int_{\partial B_{r}} u e^{-\Phi} d \mathcal{H}^{d-1} .
\end{aligned}
$$

We now set $\beta=2 c r \lambda_{m}$ so that, combining (5.34), (5.35) and (5.36) we have

$$
E(u, r) \leq 2\|\nabla \eta\|_{L^{\infty}\left(\partial B_{r}\right)} \int_{\partial B_{r}} u e^{-\Phi} d \mathcal{H}^{d-1}+\lambda_{m} \int_{B_{r}} u^{2} e^{-\Phi} d x .
$$

Now, for every $s \in(0, r]$, we have by the $W^{1,1}$ trace inequality in $B_{s}$

$$
\begin{aligned}
\int_{\partial B_{s}} u e^{-\Phi} d \mathcal{H}^{d-1} & \leq e^{-\min \Phi} C_{d}\left(\int_{B_{s}}|\nabla u| d x+\frac{1}{s} \int_{B_{s}} u d x\right) \\
& \leq e^{-\min \Phi} C_{d}\left(\frac{1}{2} \int_{B_{s}}|\nabla u|^{2} d x+\frac{1}{2}\left|\Omega_{u} \cap B_{s}\right|+c\left|\Omega_{u} \cap B_{s}\right|\right) \\
& \leq C\left(\int_{B_{s}}|\nabla u|^{2} e^{-\Phi} d x+\mu\left|\Omega_{u} \cap B_{s}\right|\right) \leq C E(u, s) \leq C E(u, r),
\end{aligned}
$$

where we have set $C=e^{-\min \Phi} C_{d} \max \left\{e^{\max \Phi}, \frac{1}{\mu}(1+2 c)\right\}$. Moreover, since the above inequality holds for every $s \in(0, r]$, we have

$$
\int_{B_{r}} u e^{-\Phi} d x=\int_{0}^{r} d s \int_{\partial B_{s}} u e^{-\Phi} d \mathcal{H}^{d-1} \leq r C E(u, r) .
$$

Finally, using the bound (5.37), we get

$$
E(u, r) \leq\left(2\|\nabla \eta\|_{L^{\infty}\left(\partial B_{r}\right)}+r^{2} c \lambda_{m}\right) C E(u, r) .
$$

Thus, the claim will follow, if we can choose $c$ such that

$$
\left(2\|\nabla \eta\|_{L^{\infty}\left(\partial B_{r}\right)}+r^{2} c \lambda_{m}\right) C<1 .
$$

We now estimate $\|\nabla \eta\|_{L^{\infty}\left(\partial B_{r}\right)}$. Notice that in $B_{2 r} \backslash B_{r}$

$$
\eta(x)=r^{2} w(x / r)+\operatorname{crh}(x / r),
$$

where $w: B_{2} \backslash B_{1} \rightarrow \mathbb{R}$ and $h: B_{2} \backslash B_{1} \rightarrow \mathbb{R}$ are the solutions to

$$
\begin{gathered}
-\Delta h+\nabla \Phi_{r} \cdot \nabla h=0 \quad \text { in } \quad B_{2} \backslash B_{1}, \quad h=0 \quad \text { on } \quad \partial B_{1}, \quad h=1 \quad \text { on } \quad \partial B_{2}, \\
-\Delta w+\nabla \Phi_{r} \cdot \nabla w=\beta \quad \text { in } \quad B_{2} \backslash B_{1}, \quad w=0 \quad \text { on } \quad \partial B_{1} \cup \partial B_{2},
\end{gathered}
$$

where $\Phi_{r}(x)=\Phi(r x)$. Thus, we have

$$
\|\nabla \eta\|_{L^{\infty}} \leq r\|\nabla w\|_{L^{\infty}}+c\|\nabla h\|_{L^{\infty}},
$$


and so, it is sufficient to estimate $\nabla w$ and $\nabla h$. First, applying Lemma 5.21 to $h$, we have

$$
\|\nabla h\|_{L^{\infty}\left(B_{2} \backslash B_{1}\right)} \leq C\|h\|_{L^{\infty}\left(B_{2} \backslash B_{1}\right)} \leq C,
$$

where the last inequality follows by the maximum principle $\left(0 \leq h \leq 1\right.$ in $\left.B_{2} \backslash B_{1}\right)$. Next, applying Lemma 5.21 to $w$, we get

$$
\|\nabla w\|_{L^{\infty}\left(B_{2} \backslash B_{1}\right)} \leq C\left(\|w\|_{L^{\infty}\left(B_{2} \backslash B_{1}\right)}+\beta\right) \leq C \beta,
$$

where the last inequality follows by Lemma 5.3. Combining the above estimates, we get

$$
\|\nabla \eta\|_{L^{\infty}\left(B_{r}\right)} \leq C(r \beta+c),
$$

which, for $c$ and $r$ small enough, implies that $E(u, r)=0$ and concludes the proof.

Another consequence of property (5.32) is that the optimal sets have finite perimeter. This fact is of independent interest but it can also be used to estimate the dimension of the singular set of the free boundary (see Subsection [5.9). The local finiteness of the perimeter was also obtained in [2] in the case of the Laplacian by a different argument. Here we use the more direct approach from [35], which is also the local version of an estimate that was used in [8] to prove that some optimal shapes have finite perimeter.

Lemma 5.23 (Local finiteness of the perimeter). Let $D \subset \mathbb{R}^{d}$ be a bounded open set and $u$ a solution of (5.1). Then $\Omega_{u}$ is a set of locally finite perimeter in D. Moreover, if $D$ is of class $C^{1,1}$, then $\Omega_{u}$ is a set of finite perimeter.

Proof. Let $x_{0} \in \partial \Omega_{u}$ and $0<\mu<\Lambda_{u}$ be fixed. Let $r>0$ be such that (5.32) holds in $D_{r}\left(x_{0}\right):=$ $B_{r}\left(x_{0}\right) \cap D$. Assume $x_{0}=0$ and $r_{0}=r$. In the sequel we denote by $C>0$ any constant, which does not depend on $t$ or $x_{0}$. Let $t \in(0,1)$ and $\eta \in C_{c}^{\infty}\left(B_{r}\right)$ be such that

$$
0 \leq \eta \leq 1, \quad \eta=1 \quad \text { in } \quad B_{r / 2}, \quad \eta=0 \quad \text { in } \quad \mathbb{R}^{d} \backslash B_{r}, \quad|\nabla \eta| \leq \frac{C}{r} .
$$

We set

$$
u_{t}:=\eta(u-t)_{+}+(1-\eta) u=\left\{\begin{aligned}
(1-\eta) u, & \text { if } u<t \\
u-t \eta, & \text { if } u \geq t
\end{aligned}\right.
$$

We can now compute on $\{u \geq t\}$

$$
\begin{aligned}
|\nabla u|^{2}-\left|\nabla u_{t}\right|^{2} & =|\nabla u|^{2}-|\nabla(u-t \eta)|^{2}=t\left(2 \nabla u \cdot \nabla \eta-t|\nabla \eta|^{2}\right) ; \\
u^{2}-u_{t}^{2} & =u^{2}-(u-t \eta)^{2}=t\left(2 u \eta+t \eta^{2}\right) .
\end{aligned}
$$

Next, on the set $\{u<t\}$, we compute

$$
\begin{aligned}
u^{2}-u_{t}^{2} & =\left(2 \eta-\eta^{2}\right) u^{2} \leq t^{2}\left(2 \eta-\eta^{2}\right) ; \\
|\nabla u|^{2}-\left|\nabla u_{t}\right|^{2} & =|\nabla u|^{2}-|\nabla(u-u \eta)|^{2}=2 \nabla u \cdot \nabla(u \eta)-|\nabla(u \eta)|^{2} \\
& =\left(2 \eta-\eta^{2}\right)|\nabla u|^{2}+2 u(1-\eta) \nabla u \cdot \nabla \eta-|\nabla \eta|^{2} u^{2} \\
& \geq \mathbb{1}_{\{\eta=1\}}|\nabla u|^{2}-2 t(1-\eta)|\nabla u \cdot \nabla \eta| .
\end{aligned}
$$

Notice that $u_{t} \in \mathcal{A}\left(u, x_{0}, r\right)$. Thus, by the optimality of $u$, we have

$$
\int_{B_{r}}\left(|\nabla u|^{2}-\lambda_{m} u^{2}\right) e^{-\Phi} d x+\mu\left|\Omega_{u} \cap B_{r}\right| \leq \int_{B_{r}}\left(\left|\nabla u_{t}\right|^{2}-\lambda_{m} u_{t}^{2}\right) e^{-\Phi} d x+\mu\left|\Omega_{u_{t}} \cap B_{r}\right| .
$$


By the above estimates, there is a constant $C$, depending only on $\mu, r, \lambda_{m}=\int_{D}|\nabla u|^{2} e^{-\Phi} d x$ and $\|\Phi\|_{L^{\infty}(D)}$ such that, for every $t \leq 1$, we have

$$
\begin{aligned}
\int_{\{0<u<t\} \cap B_{r / 2}}|\nabla u| d x & \leq \int_{\{0<u<t\} \cap B_{r / 2}}\left(|\nabla u|^{2}+1\right) d x \leq \max \{1,1 / \mu\} \int_{\{0<u<t\} \cap B_{r / 2}}\left(|\nabla u|^{2}+\mu\right) d x \\
& =\max \{1,1 / \mu\}\left(\int_{B_{r / 2}}\left(|\nabla u|^{2}-\left|\nabla u_{t}\right|^{2}\right) d x+\mu\left(\left|\Omega_{u} \cap B_{r}\right|-\left|\Omega_{u_{t}} \cap B_{r}\right|\right)\right) \leq C t .
\end{aligned}
$$

We now use the co-area formula to rewrite the above inequality as

$$
\frac{1}{t} \int_{0}^{t} \operatorname{Per}\left(\{u>s\} ; B_{r / 2}\right) d s \leq C .
$$

Hence, there is a sequence $t_{n} \rightarrow 0$ such that $\operatorname{Per}\left(\left\{u>t_{n}\right\} ; B_{r / 2}\right) d s \leq C$, which implies that $\operatorname{Per}\left(\Omega_{u} ; B_{r / 2}\right) d s \leq C$. The last claim of the lemma follows by a standard covering argument.

5.8. Blow-up sequences and blow-up limits. Let $u$ be a solution of (5.1) in the bounded open set $D \subset \mathbb{R}^{d}$. For $r>0$ and $x_{0} \in \partial \Omega_{u}$, we define the rescaled function

$$
u_{x_{0}, r}(x):=\frac{1}{r} u\left(x_{0}+r x\right) .
$$

Now since $u$ is Lipschitz continuous in some ball $B_{r_{0}}\left(x_{0}\right)$ (assume some regularity of the box if $x_{0} \in \partial D$ ) we get that every sequence $\left(u_{x_{0}, r_{n}}\right)_{n \geq 1}$ such that $r_{n} \rightarrow 0$ admits a subsequence (still denoted by $r_{n}$ ) that converges to a function $u_{0}: \mathbb{R}^{d} \rightarrow \mathbb{R}$ uniformly on every compact set $K \subset \mathbb{R}^{d}$. We say that $u_{0}$ is a blow-up limit of $u$ at $x_{0}$ and we use the notation $\mathcal{B U}_{u}\left(x_{0}\right)$ for the family of all blow-up limits of $u$ at $x_{0}$. We notice that, due to the non-degeneracy of $u$, the blow-up limits are non-trivial. Precisely, $u_{0} \neq 0$ and there is a constant $c>0$ such that $\left\|u_{0}\right\|_{L^{\infty}\left(B_{r}\right)} \geq c r$.

The following proposition is standard. For a detailed proof we refer for example to [34, Proposition 4.5].

Proposition $\mathbf{5 . 2 4}$ (Convergence of the blow-up sequences). Let $u$ be a solution of (5.1) and let $x_{0} \in \partial \Omega_{u}$. Assume moreover that $D$ is of class $C^{1,1}$ if $x_{0} \in \partial D$. Let $u_{0} \in \mathcal{B U}_{u}\left(x_{0}\right)$ and $u_{n}:=u_{x_{0}, r_{n}}$ be a blow-up sequence such that $u_{n} \rightarrow u_{0}$ locally uniformly in $\mathbb{R}^{d}$ as $n \rightarrow \infty$. Then

(1) The sequence $\left(u_{n}\right)_{n \geq 1}$ converges to $u_{0}$ strongly in $H_{l o c}^{1}\left(\mathbb{R}^{d}\right)$.

(2) The sequence of characteristic functions $\left(\mathbb{1}_{\Omega_{u_{n}}}\right)_{n>1}$ converges to $\mathbb{1}_{\Omega_{u_{0}}}$ in $L_{\text {loc }}^{1}\left(\mathbb{R}^{d}\right)$.

(3) The sequences of closed sets $\left(\bar{\Omega}_{n}\right)_{n \geq 1}$ and $\left(\Omega_{n}^{c}\right)_{n \geq 1}$ Hausdorff converge locally in $\mathbb{R}^{d}$ to $\bar{\Omega}_{0}$ and $\Omega_{0}^{c}$, respectively.

(4) If $x_{0} \in \partial \Omega_{u} \cap D$, then $u_{0}$ is a non-trivial global minimizer of the one-phase Alt-Caffarelli functional with $\Lambda=\Lambda_{u} e^{\Phi\left(x_{0}\right)}$ (see Definition 5.25 below).

If $x_{0} \in \partial \Omega_{u} \cap \partial D$, then, up to a rotation, $u_{0}$ is a non-trivial global minimizer of the one-phase constrained Alt-Caffarelli functional with $\Lambda=\Lambda_{u} e^{\Phi\left(x_{0}\right)}$.

Definition 5.25 (Global minimizers of the one-phase problem). Let $\Lambda>0$ and $u \in H_{l o c}^{1}\left(\mathbb{R}^{d}\right)$ be a non-negative function.

- We say that $u$ is a global minimizer of the one-phase Alt-Caffarelli functional with $\Lambda$, if

$$
\int_{B}|\nabla u|^{2} d x+\Lambda|\{u>0\} \cap B| \leq \int_{B}|\nabla v|^{2} d x+\Lambda|\{v>0\} \cap B|,
$$

for every ball $B \subset \mathbb{R}^{d}$ and every function $v \in H^{1}(B)$ such that $u-v \in H_{0}^{1}(B)$.

- We say that $u$ is a global minimizer of the one-phase constrained Alt-Caffarelli functional with $\Lambda$, if $\Omega_{u} \subset\left\{x_{d}>0\right\}$ and (5.38) holds for every ball $B \subset \mathbb{R}^{d}$ and every function $v \in H^{1}(B)$ such that $u-v \in H_{0}^{1}(B)$ and $\Omega_{v} \subset\left\{x_{d}>0\right\}$. 
The optimality of the blow-up limit at points $x_{0} \in D$ (Proposition 5.24 claim (4)) follows by a standard argument based on our analysis in Subsection 5.5. Below, we give the proof in the case when $x_{0}$ lies on the boundary of $D$. The idea is to straighten out the boundary of the box and to show that the function $u$ in the new coordinates satisfies an almost-minimality condition. We only give the proof of Proposition 5.24 (4) in order to show how to deal with the fact that on different scales $r$ the inclusion constraint on the set $\Omega_{u_{r}}$ changes and that at the limit the box $D$ becomes the half-space $\left\{x_{d}>0\right\}$.

Let $x_{0}=0 \in \partial \Omega_{u} \cap \partial D$. Since $D$ is $C^{1,1}$ regular, there exist $\delta>0$ and a function $g$ : $(-\delta, \delta)^{d-1} \rightarrow \mathbb{R}$ such that

$$
D \cap S Q_{\delta}=\left\{\left(x^{\prime}, x_{d}\right) \in S Q_{\delta}: g\left(x^{\prime}\right)<x_{d}\right\},
$$

where $S Q_{\delta}=(-\delta, \delta)^{d} \subset B_{r_{0}}$. Moreover, up to a rotation, we can assume that the differential $D g_{0}$ of $g$ at 0 is zero. Let $\psi: S Q_{\delta} \subset \mathbb{R}^{d} \rightarrow \mathbb{R}^{d}$ be the function that straightens out the boundary of $D$ and let $\phi:=\psi^{-1}: \psi\left(S Q_{\delta}\right) \subset \mathbb{R}^{d} \rightarrow \mathbb{R}^{d}$ be its inverse:

$$
\psi\left(x^{\prime}, x_{d}\right)=\left(x^{\prime}, x_{d}-g\left(x^{\prime}\right)\right), \quad \phi\left(x^{\prime}, x_{d}\right)=\left(x^{\prime}, x_{d}+g\left(x^{\prime}\right)\right) .
$$

We define the matrix-valued function $A=\left(a_{i j}\right)_{i j}: S Q_{\delta} \rightarrow \operatorname{Sym}_{d}^{+}(\mathbb{R})$ by

$$
A_{x}=\left(D \phi_{x}\right)^{-1}\left({ }^{t} D \phi_{x}\right)^{-1}, \quad \text { for every } \quad x \in S Q_{\delta},
$$

where ${ }^{t} D \phi_{x}$ stands for the transpose of the Jacobian matrix of $\phi$ at $x$. Note that the coefficients $a_{i j}$ are Lipschitz continuous functions and that $A_{x}$ are symmetric positive definite matrices since they are small variations of $A_{0}=I d$. For $v \in H^{1}\left(\mathbb{R}^{d}\right)$ and $r>0$ we define the functional

$$
\tilde{J}(v, r)=\int_{B_{r}}\left(a_{i j}(x) \frac{\partial v}{\partial x_{i}} \frac{\partial v}{\partial x_{j}}-\lambda_{m} v^{2}\right) e^{-\tilde{\Phi}} d x,
$$

where we have set $\tilde{\Phi}=\Phi \circ \phi$. Moreover, we set $H=\left\{\left(x^{\prime}, x_{d}\right) \in \mathbb{R}^{d}: x_{d}>0\right\}$. With an elementary change of variables we get the following result.

Lemma 5.26 (Minimality of $u$ in the straightened coordinates). Let $u$ be a solution of (5.1) and $x_{0}=0 \in \partial \Omega_{u} \cap \partial D$. Let $h>0$. There exist $c>0$ and $r_{0}>0$ such that $B_{2 r_{0}} \subset \psi\left(S Q_{\delta}\right)$ and the function $\tilde{u}=u \circ \Phi$ satisfies the minimality condition: for every $r \in\left(0, r_{0}\right)$ we have

$$
\tilde{J}(\tilde{u}, r)+\mu\left|\Omega_{\tilde{u}} \cap B_{r}\right| \leq \tilde{J}(\tilde{v}, r)+\mu\left|\Omega_{\tilde{v}} \cap B_{r}\right|
$$

for every $\tilde{v} \in H^{1}\left(B_{2 r_{0}}\right)$ such that $\tilde{u}=\tilde{v}$ on $B_{2 r_{0}} \backslash B_{r}, \Omega_{\tilde{v}} \subset H$ and where

$$
\mu=\left\{\begin{array}{l}
\mu_{+}(h, 0, c r) \quad \text { if } \quad\left|\Omega_{\tilde{u}} \cap B_{r}\right| \leq\left|\Omega_{\tilde{v}} \cap B_{r}\right| \leq\left|\Omega_{\tilde{u}} \cap B_{r}\right|+h, \\
\mu_{-}(h, 0, c r) \quad \text { if } \quad\left|\Omega_{\tilde{u}} \cap B_{r}\right|-h \leq\left|\Omega_{\tilde{v}} \cap B_{r}\right| \leq\left|\Omega_{\tilde{u}} \cap B_{r}\right| .
\end{array}\right.
$$

Proof. Let $r_{0}>0$ be such that $B_{2 r_{0}} \subset \psi\left(S Q_{\delta}\right), r \in\left(0, r_{0}\right)$ and $\tilde{v}$ such that $\tilde{u}=\tilde{v}$ on $B_{2 r_{0}} \backslash B_{r}$, $\Omega_{\tilde{v}} \subset H$. Assume that $\left|\Omega_{\tilde{u}} \cap B_{r}\right| \leq\left|\Omega_{\tilde{v}} \cap B_{r}\right| \leq\left|\Omega_{\tilde{u}} \cap B_{r}\right|+h$. We define $v \in H_{0}^{1}(D)$ by $v=\tilde{v} \circ \psi$ in $\phi\left(B_{2 r_{0}}\right)$ and $v=u$ otherwise. Let $c$ be a positive constant depending only on $\phi$ such that $\phi\left(B_{r}\right) \subset B_{c r}$. Then, it follows that $u=v$ on $D \backslash B_{c r}$. Moreover, since $\operatorname{det}\left(D \phi_{x}\right)=1$ we have $\left|\Omega_{u}\right| \leq\left|\Omega_{v}\right| \leq\left|\Omega_{u}\right|+h$. Therefore, up to chosing $r_{0}>0$ smaller (depending on $c$ ), we get

$$
J(u)+\mu_{+}(h, 0, c r)\left|\Omega_{u}\right| \leq J(v)+\mu_{+}(h, 0, c r)\left|\Omega_{v}\right| .
$$

Since we have $u=v$ on $\phi\left(B_{r}\left(x_{0}\right)\right)$, this can rewrite as

$$
\int_{\phi\left(B_{r}\right)}\left(|\nabla u|^{2}-\lambda_{m} u^{2}\right) e^{-\Phi} d x+\mu\left|\Omega_{u} \cap \phi\left(B_{r}\right)\right| \leq \int_{\phi\left(B_{r}\right)}\left(|\nabla v|^{2}-\lambda_{m} v^{2}\right) e^{-\Phi} d x+\mu\left|\Omega_{v} \cap \phi\left(B_{r}\right)\right|,
$$

where we have set $\mu=\mu_{+}(h, 0, c r)$. Now, a change of variables gives

$$
\begin{aligned}
\tilde{J}(\tilde{u}, r)+\mu\left|\Omega_{\tilde{u}} \cap B_{r}\right| & =\int_{\phi\left(B_{r}\right)}\left(|\nabla u|^{2}-\lambda_{m} u^{2}\right) e^{-\Phi} d x+\mu\left|\Omega_{u} \cap \phi\left(B_{r}\right)\right| \\
& \leq \int_{\phi\left(B_{r}\right)}\left(|\nabla v|^{2}-\lambda_{m} v^{2}\right) e^{-\Phi} d x+\mu\left|\Omega_{v} \cap \phi\left(B_{r}\right)\right|=\tilde{J}(\tilde{v}, r)+\mu\left|\Omega_{\tilde{v}} \cap B_{r}\right| .
\end{aligned}
$$


This concludes the proof.

The next Lemma states that $\tilde{u}$ is an almost-minimizer also of the functional $J$.

Lemma 5.27. Let $u$ be a solution of (5.1) and $x_{0}=0 \in \partial \Omega_{u} \cap \partial D$. Let $h>0$ be a given constant and let $c>0$ and $r_{0}>0$ be as in Lemma 5.26. Then there exists a constant $C>0$ such that $\tilde{u}=u \circ \phi$ satisfies the following almost-minimality condition: for every $r \in\left(0, r_{0}\right)$ we have

$$
J(\tilde{u}, r)+\mu\left|\Omega_{\tilde{u}} \cap B_{r}\right| \leq(1+C r) J(\tilde{v}, r)+\mu\left|\Omega_{\tilde{v}} \cap B_{r}\right|+C r \int_{B_{r}} \tilde{v}^{2} d x+C r^{d+1}
$$

for every $\tilde{v} \in H^{1}\left(B_{2 r_{0}}\right)$ such that $\tilde{u}=\tilde{v}$ on $B_{2 r_{0}} \backslash B_{r}, \Omega_{\tilde{v}} \subset H$ and where $\mu$ is as in (5.39).

Proof. Using the Lipschitz continuity of $A$ and $\Phi$, we estimate

$$
\begin{aligned}
J(\tilde{u}, r)=\tilde{J}(\tilde{u}, r)+\int_{B_{r}}\left(a_{i j}(\right. & \left.\left.x_{0}\right)-a_{i j}(x)\right) \frac{\partial \tilde{u}}{\partial x_{i}} \frac{\partial \tilde{u}}{\partial x_{j}} e^{-\Phi} d x \\
& +\int_{B_{r}}\left(a_{i j}(x) \frac{\partial \tilde{u}}{\partial x_{i}} \frac{\partial \tilde{u}}{\partial x_{j}}-\lambda_{m} \tilde{u}^{2}\right)\left(e^{-\Phi}-e^{-\tilde{\Phi}}\right) d x \leq \tilde{J}(\tilde{u}, r)+C r^{d+1},
\end{aligned}
$$

for some positive constant $C$ that does not depend on $r$. Analogously, we get the following estimate from below

$$
\begin{aligned}
J(\tilde{v}, r) & =\tilde{J}(\tilde{v}, r)+\int_{B_{r}}\left(a_{i j}\left(x_{0}\right)-a_{i j}(x)\right) \frac{\partial \tilde{v}}{\partial x_{i}} \frac{\partial \tilde{v}}{\partial x_{j}} e^{-\Phi} d x \\
& \quad+\int_{B_{r}}\left(a_{i j}(x) \frac{\partial \tilde{v}}{\partial x_{i}} \frac{\partial \tilde{v}}{\partial x_{j}}-\lambda_{m} \tilde{v}^{2}\right)\left(e^{-\Phi}-e^{-\tilde{\Phi}}\right) d x \\
\geq & \tilde{J}(\tilde{v}, r)-C r\left(\int_{B_{r}} a_{i j}(x) \frac{\partial \tilde{v}}{\partial x_{i}} \frac{\partial \tilde{v}}{\partial x_{j}} e^{-\Phi} d x+\int_{B_{r}} \tilde{v}^{2} d x\right) \\
\geq & (1-C r) \tilde{J}(\tilde{v}, r)-C r \int_{B_{r}} \tilde{v}^{2} d x .
\end{aligned}
$$

Now, using Lemma 5.26 and then combining the above estimates we get

$$
\begin{aligned}
J(\tilde{u}, r)+\mu\left|\Omega_{\tilde{u}} \cap B_{r}\right| & \leq \tilde{J}(\tilde{u}, r)+\mu\left|\Omega_{\tilde{u}} \cap B_{r}\right|+C r^{d+1} \leq \tilde{J}(\tilde{v}, r)+\mu\left|\Omega_{\tilde{v}} \cap B_{r}\right|+C r^{d+1} \\
& \leq \frac{1}{1-C r}\left(J(\tilde{v}, r)+C r \int_{B_{r}} \tilde{v}^{2} d x\right)+\mu\left|\Omega_{\tilde{v}} \cap B_{r}\right|+C r^{d+1},
\end{aligned}
$$

which concludes the proof.

We are now in position to prove the claim (4) of Proposition 5.24 in the constrained case.

Proof of Proposition 5.24 (4). Let $x_{0}=0 \in \partial \Omega_{u} \cap \partial D$ and let $u_{0} \in \mathcal{B U}_{u}\left(x_{0}\right)$ be the blow-up limit of the sequence $u_{n}(x)=r_{n}^{-1} u\left(r_{n} x\right)$, where $\left(r_{n}\right)_{n}$ is some fixed sequence decreasing to 0 . Let $v \in H^{1}\left(B_{r}\right)$ be such that $u_{0}-v \in H_{0}^{1}\left(B_{r}\right)$ and $\Omega_{v} \subset H$. We define $v_{n}=v+\tilde{u}_{n}-u_{0} \in H^{1}\left(\mathbb{R}^{d}\right)$, where we have set $\tilde{u}_{n}(x)=r_{n}^{-1} \tilde{u}\left(r_{n} x\right)$. Note that the sequence $\left(u_{n}\right)_{n}$ and $\left(\tilde{u}_{n}\right)_{n}$ converge to the same limit $u_{0}$ since the function $\phi$ is $C^{1,1}$ regular. Moreover, since $u_{0}-v \in H_{0}^{1}\left(B_{r}\right)$ we have $\tilde{u}_{n}-v_{n} \in H_{0}^{1}\left(B_{r}\right)$ and hence $\tilde{u}-v_{n}^{r_{n}} \in H_{0}^{1}\left(B_{r r_{n}}\right)$, where we write $v_{n}^{r_{n}}(x)=r_{n} v_{n}\left(x / r_{n}\right)$. Note that we have $\Omega_{v_{n}^{r_{n}}} \subset H$. We set $h_{n}=\left|B_{r r_{n}}\right|$ and assume that $\left|\Omega_{\tilde{u}} \cap B_{r r_{n}}\right| \leq\left|\Omega_{v_{n}^{r_{n}}} \cap B_{r r_{n}}\right| \leq$ $\left|\Omega_{\tilde{u}} \cap B_{r r_{n}}\right|+h_{n}$. Now we set $\mu=\mu_{+}\left(h_{n}, 0, r\right)$ and $\Phi_{n}(x)=\Phi\left(r_{n} x\right)$ and we apply Lemma 5.27 to the test function $v_{n}^{r_{n}}$ to estimate

$$
\begin{aligned}
\int_{B_{r}} & \left(\left|\nabla \tilde{u}_{n}\right|^{2}-r_{n}^{2} \lambda_{m} \tilde{u}_{n}^{2}\right) e^{-\Phi_{n}(x)} d x+\mu\left|\Omega_{\tilde{u}_{n}} \cap B_{r}\right|=\frac{1}{r_{n}^{d}}\left(J\left(\tilde{u}, r r_{n}\right)+\mu\left|\Omega_{\tilde{u}} \cap B_{r r_{n}}\right|\right) \\
& \leq \frac{1}{r_{n}^{d}}\left(\left(1+C r r_{n}\right) J\left(v_{n}^{r_{n}}, r r_{n}\right)+\mu\left|\Omega_{v_{n}^{r_{n}}} \cap B_{r r_{n}}\right|+C r r_{n} \int_{B_{r r_{n}}}\left(v_{n}^{r_{n}}\right)^{2} d x+C\left(r r_{n}\right)^{d+1}\right) \\
& =\left(1+C r r_{n}\right) \int_{B_{r}}\left(\left|\nabla v_{n}\right|^{2}-r_{n}^{2} \lambda_{m} v_{n}^{2}\right) e^{-\Phi_{n}(x)} d x+\mu\left|\Omega_{v_{n}} \cap B_{r}\right|+C r r_{n}^{3} \int_{B_{r}} v_{n}^{2} d x+C r^{d+1} r_{n} .
\end{aligned}
$$


By Proposition 5.24 (1) the sequence $\tilde{u}_{n}$ (resp. $v_{n}$ ) strongly converges in $H^{1}\left(B_{r}\right)$ to $u_{0}$ (resp. $v)$ and the sequence of characteristic functions $\left(\mathbb{1}_{\Omega_{\tilde{u}_{n}}}\right)_{n \geq 1}$ (resp. $\left.\left(\mathbb{1}_{\Omega_{v_{n}}}\right)_{n \geq 1}\right)$ converges to $\mathbb{1}_{\Omega_{u_{0}}}$ (resp. $\left.\mathbb{1}_{\Omega_{v}}\right)$. Moreover, $\mu=\mu_{+}\left(h_{n}, 0, r\right)$ tends to $\Lambda_{u}$ as $r_{n} \rightarrow 0$ by Theorem 5.16. Therefore, passing at the limit in the above inequality and then multiplying by $e^{\Phi\left(x_{0}\right)}$ gives the claim.

Remark 5.28 (Lebesgue density on the free boundary). For every $\gamma \in[0,1]$ we define

$$
\Omega_{u}^{(\gamma)}:=\left\{x \in \mathbb{R}^{d}: \lim _{r \rightarrow 0} \frac{\left|\Omega_{u} \cap B_{r}(x)\right|}{\left|B_{r}\right|}=\gamma\right\}
$$

We notice that, as a consequence of Proposition [5.24, we get that

$$
\partial \Omega_{u} \cap D \cap \Omega_{u}^{(0)}=\emptyset \quad \text { and } \quad \partial \Omega_{u} \cap D \cap \Omega_{u}^{(1)}=\emptyset .
$$

The first equality follows by the non-degeneracy of $u$, while the second one follows from the fact that all the blow-up limits vanish in zero and are global solutions of the Alt-Caffarelli problem.

5.9. Regularity of the free boundary. In this section we prove Theorem 1.5 (4) and (6), and Theorem 1.2 (4) and (6). We first show that the optimality condition $|\nabla u|^{2}=\Lambda_{u} e^{\Phi}$ on the free boundary $\partial \Omega_{u} \cap D$ and $|\nabla u|^{2} \geq \Lambda_{u} e^{\Phi}$ on $\partial \Omega_{u} \cap \partial D$ holds in the viscosity sense (Lemma 5.30). We will then decompose the free boundary into regular and singular parts (Definition 5.34) and we will show that the regular part is $C^{1, \alpha}$ regular (Proposition 5.35).

Definition 5.29 (Optimality condition in viscosity sense). Let $D$ be an open set and $u: D \rightarrow \mathbb{R}$ be continuous, that is, $u \in C(D)$.

- We say that $\varphi \in C(D)$ touches $u$ by below (resp. by above) at $x_{0} \in D$ if $\varphi\left(x_{0}\right)=u\left(x_{0}\right)$ and $\varphi \leq u($ resp. $\varphi \geq u)$ in a neighborhood of $x_{0}$.

- Let $\Lambda$ be a non-negative function on $\bar{D}$ and assume that $u$ is non-negative. We say that $u$ satisfies the boundary condition

$$
|\nabla u|=\sqrt{\Lambda} \quad \text { on } \quad \partial \Omega_{u} \cap D
$$

in viscosity sense if, for every $\varphi \in C^{2}(D)$ such that $\varphi^{+}$touches $u$ by below (resp. by above) at some $x_{0} \in \partial \Omega_{u} \cap D$, we have $|\nabla \varphi|\left(x_{0}\right) \leq \sqrt{\Lambda}\left(\right.$ resp. $\left.|\nabla \varphi|\left(x_{0}\right) \geq \sqrt{\Lambda}\right)$ ).

Analogously, we say that $u$ satisfies the boundary condition

$$
|\nabla u| \geq \sqrt{\Lambda} \text { on } \partial \Omega_{u} \cap \partial D
$$

in viscosity sense if, for every $\varphi \in C^{2}(D)$ such that $\varphi^{+}$touches $u$ by above at some $x_{0} \in \partial \Omega_{u} \cap \partial D$, we have $|\nabla \varphi|\left(x_{0}\right) \geq \sqrt{\Lambda}$.

Lemma 5.30 (Optimality condition on the free boundary). Let $D \subset \mathbb{R}^{d}$ be a bounded open set of class $C^{1,1}$ and let $u$ be a solution of (5.1). Then $u$ is a solution of the problem

$$
\left\{\begin{array}{l}
-\operatorname{div}\left(e^{-\Phi} u\right)=\lambda_{m} u e^{-\Phi} \quad \text { in } \Omega_{u}, \\
|\nabla u|=\sqrt{\Lambda_{u} e^{\Phi}} \text { on } \partial \Omega_{u} \cap D, \\
|\nabla u| \geq \sqrt{\Lambda_{u} e^{\Phi}} \quad \text { on } \partial \Omega_{u} \cap \partial D,
\end{array}\right.
$$

where the boundary conditions hold in viscosity sense.

To prove the optimality condition we will need the following result.

Lemma 5.31. Let $u \in H^{1}\left(\mathbb{R}^{d}\right)$ be a non-trivial, continuous and one-homogeneous function (in the sense that $u(t x)=t u(x)$ for every $t>0)$ such that $u(0)=0$. Assume moreover that $u$ is harmonic in the set $\Omega_{u}$. If $\Omega_{u} \subset\left\{x_{d}>0\right\}$ then $\Omega_{u}=\left\{x_{d}>0\right\}$, while if $\Omega_{u} \supset\left\{x_{d}>0\right\}$ then either $\Omega_{u}=\left\{x_{d}>0\right\}$ or $\Omega_{u}=\left\{x_{d} \neq 0\right\}$.

Proof. Set $S=\Omega_{u} \cap \partial B_{1}$ and denote by $C_{S}=\{r \theta: \theta \in S, r>0\}$ the cone generated by $S$. Since $u$ is a one-homogeneous function and is solution of

$$
\Delta u=0 \quad \text { in } C_{S}, \quad u=0 \text { on } \partial C_{S},
$$


it follows that the trace $\varphi=u_{\left.\right|_{\partial B_{1}}}$ is a solution of

$$
-\Delta_{\mathbb{S}^{d-1}} \varphi=(d-1) \varphi \quad \text { in } \quad S, \quad \varphi=0 \quad \text { on } \partial S .
$$

Therefore $\varphi$ is a first eigenfunction of $-\Delta_{\mathbb{S}^{d-1}}$ in $S$ (because $\varphi>0$ in $S$ ) and hence $\lambda_{1}(S)=d-1$. Note also that $\varphi_{1}=\left(x_{d}\right)_{+}$is the first eigenfunction on the set $S_{+}=\left\{x_{d}>0\right\} \cap \partial B_{1}$ with eigenvalue $\lambda_{1}\left(S_{+}\right)=d-1$.

Firstly, assume that $S \subset S_{+}$. Then $u \in H_{0}^{1}\left(S_{+}\right)$and by the variational characterization of $\lambda_{1}\left(S_{+}\right)$we have

$$
\frac{\int_{\partial B_{1}}|\nabla \varphi|^{2} d \mathcal{H}^{d-1}}{\int_{\partial B_{1}} \varphi^{2} d \mathcal{H}^{d-1}}=\lambda_{1}(S)=\lambda_{1}\left(S_{+}\right) \leq \frac{\int_{\partial B_{1}}|\nabla(\varphi+t \psi)|^{2} d \mathcal{H}^{d-1}}{\int_{\partial B_{1}}(\varphi+t \psi)^{2} d \mathcal{H}^{d-1}}
$$

for every $\psi \in H_{0}^{1}\left(S_{+}\right)$and $t \in \mathbb{R}$. This gives that $\varphi$ is solution of

$$
-\Delta_{\mathbb{S}^{d-1}} \varphi=\lambda_{1}\left(S_{+}\right) \varphi \quad \text { in } \quad S_{+}, \quad \varphi=0 \quad \text { on } \quad \partial S_{+},
$$

that is, $\varphi$ is the first eigenfunction on $S_{+}$. Since $\lambda_{1}\left(S_{+}\right)$is simple (because $S_{+}$is connected) is follows that $\varphi=c\left(x_{d}\right)_{+}$for some $c>0$. In particular, $\{\varphi>0\}=S_{+}$and hence $\Omega_{u}=\left\{x_{d}>0\right\}$ by one-homogeneity of $u$.

Assume now that $S \supset S_{+}$and write $S=S_{0} \sqcup S_{1}$, where $S_{0}$ is the connected component of $S$ which contains $S_{+}$. If $S_{1} \neq \emptyset$, then it follows by the preceding step that $S_{1}=S_{-}:=\left\{x_{d}<\right.$ $0\} \cap \partial B_{1}$; hence $S=S_{+} \cup S_{-}$and $\Omega_{u}=\left\{x_{d} \neq 0\right\}$. Now, if $S_{1}=\emptyset$, then $S=S_{0}$ is connected. Moreover, $\varphi_{1} \in H_{0}^{1}(S)$ and using the variational characterization of $\lambda_{1}(S)$ it follows that $\varphi_{1}$ is the first eigenfunction in $S$. Then $\varphi_{1}>0$ in $S$ (since $S$ is connected) which proves that $S=S_{+}$.

Proof of Lemma 5.30. From Proposition 5.18 it follows that $u$ is continuous in $D$. We only have to prove that $u$ satisfies the two boundary conditions in the viscosity sense. We first show that $|\nabla u|=\sqrt{\Lambda_{u} e^{\Phi}}$ holds on $\partial \Omega_{u} \cap D$. Let $\varphi \in C^{2}(D)$ a function such that $\varphi^{+}$touches $u$ by below at $x_{0} \in \partial \Omega_{u} \cap D$. Let $r_{n}$ be an infinitesimal sequence and

$$
u_{n}(x)=\frac{1}{r_{n}} u\left(x_{0}+r_{n} x\right) \text { and } \varphi_{n}(x)=\frac{1}{r_{n}} \varphi\left(x_{0}+r_{n} x\right) .
$$

Up to a subsequence, $u_{n}$ converges locally uniformly to some $u_{0} \in \mathcal{B} \mathcal{U}_{u}\left(x_{0}\right)$, while $\varphi_{n}$ converges to $\varphi_{0}(x):=x \cdot \nabla \varphi\left(x_{0}\right)$. Up to a change of coordinates, we may suppose that $\nabla \varphi\left(x_{0}\right)=\left|\nabla \varphi\left(x_{0}\right)\right| e_{d}$. If $\left|\nabla \varphi\left(x_{0}\right)\right|=0$, then $\left|\nabla \varphi\left(x_{0}\right)\right| \leq \sqrt{\Lambda}$, where we have set $\Lambda=\Lambda_{u} e^{\Phi\left(x_{0}\right)}$, and we are done. Otherwise, we have $u_{0}>0$ in the half-space $\left\{x_{d}>0\right\}$ since $u_{0} \geq \varphi_{0}$. Moreover, $u_{0}$ is a one-homogeneous function by Lemma 5.38 and it follows that $\Omega_{u_{0}}=\left\{x_{d}>0\right\}$ by Lemma 5.31, because the case $\Omega_{u_{0}}=\left\{x_{d} \neq 0\right\}$ is ruled out (by (4) in Proposition 5.24 or Remark 5.28). Moreover, $u_{0}$ is a local minimizer of the Alt-Caffarelli functional for $\Lambda$ by Proposition 5.24 and hence satisfies (in the classical sense) the optimality condition

$$
\left|\nabla u_{0}\right|=\sqrt{\Lambda} \quad \text { on } \quad\left\{x_{d}=0\right\}
$$

(see [2, Theorem 2.5]). This implies that $u_{0}=\sqrt{\Lambda} x_{d}^{+}$. To see this, note that the boundary condition implies that $v$ defined by $v=u_{0}$ in $\left\{x_{d}>0\right\}$ and $v=\sqrt{\Lambda} x_{d}$ in $\left\{x_{d} \leq 0\right\}$ is harmonic in $\mathbb{R}^{d}$; hence $v=\sqrt{\Lambda} x_{d}$ by uniqueness of the solution to Cauchy problem for the Laplacian. Finally, since $u_{0} \geq \varphi_{0}$ we have $\sqrt{\Lambda} \geq\left|\nabla \varphi_{0}\right|(0)=|\nabla \varphi|\left(x_{0}\right)$. The proof if now $\varphi^{+}$touches $u$ from above is similar. In this case we have $\Omega_{u_{0}} \subset\left\{x_{d}>0\right\}$ since $u_{0} \leq \varphi_{0}^{+}$, and hence $\Omega_{u}=\left\{x_{d}>0\right\}$ by Lemma 5.31. Notice that $u_{0}$ is a non trivial function by the non-degeneracy property in Lemma 5.22.

Suppose now that $\varphi^{+}$touches $u$ from above at $x_{0} \in \partial \Omega_{u} \cap \partial D$ and consider $u_{n}$ and $\varphi_{n}$ defined in (5.41). By Proposition 5.24, $u_{n}$ converges to a local minimizer $u_{0}$ of the Alt-Caffarelli functional with $\Lambda=\Lambda_{u} e^{\Phi\left(x_{0}\right)}$ in the half-space $\left\{x_{d}>0\right\}$ (up to a change of coordinates). Therefore, the sequence $u_{0 n}:=r_{n}^{-1} u_{0}\left(r_{n} x\right)$ converges to a limit $u_{00}$ which is a solution of the constrained AltCaffarelli problem in $\left\{x_{d}>0\right\}$ and is one-homogenous (see Remark 5.32). Therefore, $\Omega_{u_{00}}=$ $\left\{x_{d}>0\right\}$ by Lemma 5.31 and $u_{00}$ satisfies the optimality condition

$$
\left|\nabla u_{00}\right| \geq \sqrt{\Lambda} \quad \text { on } \quad\left\{x_{d}=0\right\} .
$$


This implies that $u_{00}(x)=\alpha x_{d}^{+}$, for some $\alpha \geq \sqrt{\Lambda}$, and thus that $\sqrt{\Lambda} \leq \alpha \leq\left|\nabla \varphi_{0}\right|(0)=|\nabla \varphi|\left(x_{0}\right)$ (since $u_{00} \leq \varphi_{0}^{+}$).

Remark 5.32. The homogeneity of the blow-up limits of the (local) minimizers of the AltCaffarelli functional was first obtained by Weiss in [40]. In the case of the constrained problem, when the solution $u$ is optimal only among the functions with support in $\left\{x_{d}>0\right\}$, the Weiss formula can still be applied because the one-homogeneous extensions of $u$ are admissible competitors. Thus, the blow-up limits in this case are still one-homogeneous. We refer for instance to [37, Proposition 4.3] and Lemma 5.37 below.

Remark 5.33 (On the Alt-Caffarelli optimality condition). Using an argument based on an internal variation of the boundary as in [2, Theorem 2.5] we can get in a weak sense the optimality boundary condition given in Lemma [5.30, namely: for every $x_{0} \in \partial \Omega_{u} \cap D, r>0$ such that $B_{r}\left(x_{0}\right) \subset D$ and $\xi \in C_{0}^{\infty}\left(B_{r}\left(x_{0}\right), \mathbb{R}^{d}\right)$ we have

$$
\lim _{\varepsilon \downarrow 0} \int_{\partial\{u>\varepsilon\}}\left(|\nabla u|^{2}-\Lambda_{u} e^{\Phi}\right) e^{-\Phi} \xi \cdot \nu d \mathcal{H}^{d-1}=0,
$$

while for every $x_{0} \in \partial \Omega_{u} \cap \partial D, r>0$ such that $D_{r}\left(x_{0}\right)$ is connected and every $\xi \in C_{0}^{\infty}\left(B_{r}\left(x_{0}\right), \mathbb{R}^{d}\right)$ such that $(I d+\xi)^{-1}\left(D_{r}\left(x_{0}\right)\right) \subset D_{r}\left(x_{0}\right)$ we have

$$
\lim _{\varepsilon \downarrow 0} \int_{\partial\{u>\varepsilon\}}\left(|\nabla u|^{2}-\Lambda_{u} e^{\Phi}\right) e^{-\Phi} \xi \cdot \nu d \mathcal{H}^{d-1} \geq 0 .
$$

Definition 5.34 (Regular and singular parts of the free boundary). We say that $x_{0} \in \partial \Omega_{u}$ is a regular point if there exists a blow-up $u_{0} \in \mathcal{B U}_{u}\left(x_{0}\right)$ of the form

$$
\begin{array}{ll}
u_{0}(x)=\sqrt{\Lambda_{u} e^{\Phi\left(x_{0}\right)}}(x \cdot \nu)_{+} & \text {if } \quad x_{0} \in \partial \Omega_{u} \cap D, \\
u_{0}(x)=q(x \cdot \nu)_{+} & \text {if } \quad x_{0} \in \partial \Omega_{u} \cap \partial D,
\end{array}
$$

where $\nu \in \partial B_{1}$ is some unit vector and $q$ is a constant such that $q \geq \sqrt{\Lambda_{u} e^{\Phi\left(x_{0}\right)}}$.

We denote by $\operatorname{Reg}\left(\partial \Omega_{u} \cap D\right)$ the set of regular points (the regular part of the free boundary) in $D$, and by $\operatorname{Reg}\left(\partial \Omega_{u}\right)$ the set of all regular points of $\partial \Omega_{u}$. We define the singular part of the boundary as $\operatorname{Sing}\left(\partial \Omega_{u}\right):=\partial \Omega_{u} \backslash \operatorname{Reg}\left(\partial \Omega_{u}\right)$ and $\operatorname{Sing}\left(\partial \Omega_{u} \cap D\right)=\partial \Omega_{u} \backslash \operatorname{Reg}\left(\partial \Omega_{u} \cap D\right)$.

Proposition 5.35 (Regularity of the free boundary). Suppose that $u$ is a solution of (5.1) in the bounded open set $D \subset \mathbb{R}^{d}$. Then, we have:

(1) $\operatorname{Reg}\left(\partial \Omega_{u} \cap D\right)$ is locally the graph of a $C^{1, \alpha}$ function for any $\alpha<1$;

(2) the reduced boundary $\partial^{*} \Omega_{u} \cap D$ is contained in $\operatorname{Reg}\left(\partial \Omega_{u} \cap D\right)$;

(3) $\mathcal{H}^{d-1}\left(\operatorname{Sing}\left(\partial \Omega_{u} \cap D\right)\right)=0$; moreover, if $d \leq 4$, then $\operatorname{Reg}\left(\partial \Omega_{u} \cap D\right)=\emptyset$.

If $D$ is a $C^{1,1}$ regular domain, then:

(4) $\operatorname{Reg}\left(\partial \Omega_{u}\right)$ is locally the graph of a $C^{1,1 / 2}$ regular function;

(5) $\partial \Omega_{u} \cap \partial D \subset \operatorname{Reg}\left(\partial \Omega_{u}\right)$;

(6) $\operatorname{Reg}\left(\partial \Omega_{u} \cap D\right) \subset \operatorname{Reg}\left(\partial \Omega_{u}\right)$ and $\operatorname{Sing}\left(\partial \Omega_{u}\right)=\operatorname{Sing}\left(\partial \Omega_{u} \cap D\right)$.

Proof. By Lemma 5.30, $u$ is a viscosity solution of (5.40). Let $x_{0} \in \partial \Omega_{u}$ be a regular point. Then, for some $r>0$ small enough the function $u_{r, x_{0}}=\frac{1}{r} u\left(x_{0}+r x\right)$ is also a viscosity solution and is $\varepsilon$-flat in the sense of [21]. Applying the results of De Silva [21] (in the case when $x_{0} \in D$ ) and Chang-Lara-Savin [18] (if $x_{0} \in \partial D$ ), we get the claims (1) and (4).

We next prove (2) and (3). Let $x_{0} \in \partial \Omega_{u} \cap D$ and $u_{n}:=u_{x_{0}, r_{n}}$ be a blow-up sequence at $x_{0}$ converging to some $v \in \mathcal{B U}_{u}\left(x_{0}\right)$ such that $\mathbb{1}_{\Omega_{u_{n}}}$ converges in $L_{l o c}^{1}\left(\mathbb{R}^{d}\right)$ to $\mathbb{1}_{\Omega_{v}}$. If $x_{0} \in \partial^{*} \Omega_{u} \cap D$, then $\Omega_{v}$ is a half-plane of the form $H=\left\{x \in \mathbb{R}^{d}: x \cdot \nu>0\right\}$ for some $\nu \in \partial B_{1}$. Without loss of generality, we assume $\nu=e_{d}$. On the other, hand $v$ is a solution to the Alt-Caffarelli problem with $\Lambda=\Lambda_{u} e^{\Phi\left(x_{0}\right)}$. Thus $v$ is harmonic in $H$ and zero on $\partial H$, so it is smooth up to the boundary of $H$. Now, the optimality condition $|\nabla v|=\sqrt{\Lambda}$ on $\partial H$ and the unique continuation 
of harmonic functions in the half-plane imply that $v(x)=\sqrt{\Lambda} x_{d}^{+}$, which proves (2). Now, since $\Omega_{u}$ has (locally) finite perimeter in $D$, the Federer Theorem and Remark 5.28 give that

$$
\mathcal{H}^{d-1}\left(\partial \Omega_{u} \cap D \backslash\left(\partial^{*} \Omega_{u}\right)\right)=\mathcal{H}^{d-1}\left(\partial \Omega_{u} \cap D \backslash\left(\partial^{*} \Omega_{u} \cup \Omega_{u}^{(0)} \cup \Omega_{u}^{(1)}\right)\right)=0,
$$

which proves that $\mathcal{H}^{d-1}\left(\operatorname{Sing}\left(\partial \Omega_{u} \cap D\right)\right)=0$. We now prove the second claim of (3). As above, let $x_{0} \in \partial \Omega_{u} \cap D$ and $v=\lim _{n \rightarrow \infty} u_{r_{n}, x_{0}}$ be a blow-up limit of $u$ at $x_{0}$. Then $v$ is a solution of the Alt-Caffarelli problem problem. Let $\rho_{n} \rightarrow 0$ and $v_{\rho_{n}}(x)=\frac{1}{\rho_{n}} v\left(x \rho_{n}\right)$ be a sequence that converges locally uniformly to a function $v_{0}$. Since $d \leq 4$, we have that the free boundary $\partial \Omega_{v}$ is $C^{1, \alpha}$ and $v_{0}$ is of the form $v_{0}(x)=\sqrt{\Lambda}(x \cdot \nu)_{+}$for some $\nu \in \partial B_{1}$ (see 2 for $d=2$, 16 for $d=3$ and 31] for $d=4)$. Now since, for fixed $n>0$, we have that $v_{\rho_{n}}=\lim _{m \rightarrow \infty} u_{\rho_{n} r_{m}, x_{0}}$, we can choose a diagonal sequence $u_{R_{n}, x_{0}}$, where $R_{n}=\rho_{n} r_{m(n)}$, such that $v_{0}=\lim _{n \rightarrow \infty} u_{R_{n}, x_{0}}$. This proves that $x_{0}$ is a regular point.

The claim (5) follows by the same diagonal sequence argument. This time $x_{0} \in \partial D$ and the blow-up $v$ is a solution of the constrained Alt-Caffarelli problem in $\left\{x_{d}>0\right\}$. Thus, the blow-up $v_{0}$ of $v$ is one-homogeneous solution of the constrained problem for $\Lambda=\Lambda_{u} e^{\Phi\left(x_{0}\right)}$. This implies (in any dimension) that $v_{0}(x)=q x_{d}^{+}$for some $q \geq \sqrt{\Lambda}$ (see [37, Proposition 4.3]).

Finally, (6) follows by the definition of the regular part and claim (5).

Remark 5.36 (On the higher regularity of the free boundary). The smoothness of the free boundary can be improved under an additional regularity assumption on $\Phi$. Indeed, if $\nabla \Phi \in$ $C^{k+1, \alpha}\left(D ; \mathbb{R}^{d}\right)$ for some $k \geq 1$ and $\alpha \in(0,1)$, then by [33, Theorem 1], $\operatorname{Reg}\left(\partial \Omega^{*}\right) \cap D$ is locally a graph of a $C^{k+1, \alpha}$ function.

5.10. Monotonicity formula and some further estimates on the dimension of the singular set. This section is dedicated to the estimates on the dimension of the singular set (Theorem 1.5 (5) and Theorem $1.2(5)$ ). The main ingredient is a monotonicity formula that implies the homogeneity of the blow-up limits at any free boundary point $x_{0} \in \partial \Omega_{u} \cap D$.

Let $u$ be a solution (5.1) and $\Lambda_{u}$ be the constant given by Theorem 5.12. We define the Weiss-type boundary adjusted energy as

$$
W\left(u, \Phi, x_{0}, r\right)=\frac{1}{r^{d}} \int_{B_{r}\left(x_{0}\right)}|\nabla u|^{2} e^{-\Phi} d x-\frac{1}{r^{d+1}} \int_{\partial B_{r}\left(x_{0}\right)} u^{2} e^{-\Phi} d \mathcal{H}^{d-1}+\frac{\Lambda_{u}}{r^{d}}\left|\Omega_{u} \cap B_{r}\left(x_{0}\right)\right| .
$$

Lemma 5.37 (Weiss monotonicity formula). Let $u$ be a solution (5.1) in the bounded open set $D$. Then, for every $x_{0} \in \partial \Omega_{u} \cap D$ and every $0<r<\operatorname{dist}\left(x_{0}, \partial D\right)$, the function $W$ satisfies the differential inequality

$$
\frac{d}{d r} W\left(u, \Phi, x_{0}, r\right) \geq \frac{2 e^{-\max \Phi}}{r^{d+2}} \int_{\partial B_{r}\left(x_{0}\right)}|\nabla u \cdot x-u|^{2} d \mathcal{H}^{d-1}-C,
$$

where $C>0$ is a constant depending only on $\lambda_{m}, \Phi, L:=\|\nabla u\|_{L^{\infty}}$ and the dimension $d$.

Proof. We first prove the claim when $x_{0} \in \partial \Omega_{u} \cap D$. Assume $x_{0}=0$. We set

$$
\begin{gathered}
H(r):=\int_{\partial B_{r}} u^{2} e^{-\Phi} d \mathcal{H}^{d-1} \quad \text { and } \quad D(r):=\int_{B_{r}}|\nabla u|^{2} e^{-\Phi} d x, \\
H_{\Phi}(r):=\int_{\partial B_{r}}(\nu \cdot \nabla \Phi) u^{2} e^{-\Phi} d \mathcal{H}^{d-1} \quad \text { and } \quad D_{\Phi}(r):=\int_{B_{r}}\left(|\nabla u|^{2}-\lambda_{m} u^{2}\right)(x \cdot \nabla \Phi) e^{-\Phi} d x,
\end{gathered}
$$

where $\nu(x)=x / r$ is the exterior normal to the sphere $\partial B_{r}$ at $x$. As in Proposition A.1 (notice that in Proposition $\$ .1 D_{\Phi}$ is defined differently) we have

$$
D^{\prime}(r)=\int_{\partial B_{r}}|\nabla u|^{2} e^{-\Phi} d \mathcal{H}^{d-1} \quad \text { and } \quad H^{\prime}(r)=\frac{d-1}{r} H(r)+2 D(r)-2 \lambda_{m} \int_{B_{r}} u^{2} e^{-\Phi} d x-H_{\Phi}(r) .
$$

Let $\phi_{\varepsilon}$ be a radially decreasing function such that

$$
0 \leq \phi_{\varepsilon} \leq 1 \text { in } B_{r}, \quad \phi_{\varepsilon}=1 \text { in } B_{r(1-\varepsilon)}, \quad \phi_{\varepsilon}=0 \text { on } \partial B_{r} \quad \text { and } \quad\left|\nabla \phi_{\varepsilon}\right| \leq C(r \varepsilon)^{-1} \text {. }
$$


As in Step 2 of the proof of Proposition A.1, the optimality condition $\delta J(u)[\xi]=\Lambda_{u} \int_{\Omega_{u}} \operatorname{div} \xi d x$, applied to the vector field $\xi(x)=x \phi_{\varepsilon}(x)$, gives that

$$
\begin{aligned}
\Lambda_{u}\left(d\left|\Omega_{u} \cap B_{r}\right|-r \mathcal{H}^{d-1}\left(\Omega_{u} \cap \partial B_{r}\right)\right)= & -(d-2) D(r)+r D^{\prime}(r)-2 r \int_{\partial B_{r}}\left(\partial_{\nu} u\right)^{2} e^{-\Phi} d \mathcal{H}^{d-1} \\
& +\lambda_{m}\left(d \int_{B_{r}} u^{2} e^{-\Phi} d x-r \int_{\partial B_{r}} u^{2} e^{-\Phi} d \mathcal{H}^{d-1}\right)+r D_{\Phi}(r),
\end{aligned}
$$

where $\partial_{\nu} u:=\nu \cdot \nabla u$. We now calculate

$$
\begin{aligned}
\frac{d}{d r} W\left(u, \Phi, x_{0}, r\right)= & \frac{1}{r^{d}} D^{\prime}(r)-\frac{d}{r^{d+1}} D(r)-\frac{1}{r^{d+1}} H^{\prime}(r)+\frac{d+1}{r^{d+2}} H(r) \\
& +\frac{\Lambda_{u}}{r^{d+1}}\left(r \mathcal{H}^{d-1}\left(\Omega_{u} \cap \partial B_{r}\right)-d\left|\Omega_{u} \cap B_{r}\right|\right) \\
= & \frac{2}{r^{d+2}} \int_{\partial B_{r}}|\nabla u \cdot x-u|^{2} e^{-\Phi} d \mathcal{H}^{d-1}+\frac{1}{r^{d+1}} H_{\Phi}(r)-\frac{1}{r^{d}} D_{\Phi}(r) \\
& \quad-\frac{\lambda_{m}}{r^{d+1}}\left((d+2) \int_{B_{r}} u^{2} e^{-\Phi} d x-r \int_{\partial B_{r}} u^{2} e^{-\Phi} d \mathcal{H}^{d-1}\right) \\
\geq & \frac{2 e^{-\max \Phi}}{r^{d+2}} \int_{\partial B_{r}}|\nabla u \cdot x-u|^{2} d \mathcal{H}^{d-1}-C,
\end{aligned}
$$

which gives the claim if $x_{0} \in \partial \Omega_{u} \cap D$ and $r<\operatorname{dist}\left(x_{0}, \partial D\right)$.

Lemma 5.38 (Homogeneity of the blow-up limits). Let $u$ be a solution (5.1) in the bounded open set $D$ and let $x_{0} \in \partial \Omega_{u}$. Then every blow-up limit $u_{0} \in \mathcal{B U}_{u}\left(x_{0}\right)$ is one-homogeneous.

Proof. Let $x_{0}=0$ and $W(u, \Phi, r):=W\left(u, \Phi, x_{0}, r\right)$. Recall that $u_{r}(x)=\frac{1}{r} u(r x)$ and $\Phi_{r}(x)=$ $\Phi(r x)$. We first notice that for every $r>0$ and $s>0$ such that $r s \leq \operatorname{dist}\left(x_{0}, \partial D\right)$ we have

$$
W\left(u_{r}, \Phi_{r}, s\right)=W(u, \Phi, r s) .
$$

Moreover, since the function $r \mapsto W(u, \Phi, t)+C r$ is monotone, the limit

$$
W(u, \Phi, 0):=\lim _{r \rightarrow 0^{+}} W(u, \Phi, r)
$$

exists (and is finite due to the Lipschtz continuity of $u$ ). On the other hand, for every blow-up sequence $u_{r_{n}}$ with blow-up limit $u_{0}$, we have

$$
W\left(u_{0}, \Phi(0), s\right)=\lim _{n \rightarrow \infty} W\left(u_{r_{n}}, \Phi_{r_{n}}(0), s\right)=\lim _{n \rightarrow \infty} W\left(u, \Phi(0), r_{n} s\right)=W(u, \Phi(0), 0) .
$$

Thus, the function

$$
s \mapsto \frac{1}{s^{d}} \int_{B_{s}}\left|\nabla u_{0}\right|^{2} d x-\frac{1}{s^{d+1}} \int_{\partial B_{s}} u_{0}^{2} d \mathcal{H}^{d-1}+\frac{\Lambda_{u} e^{\Phi(0)}}{s^{d}}\left|\Omega_{u_{0}} \cap B_{s}\right|,
$$

is constant. Now, by [40] (or, simply by applying (5.44) to $u=u_{0}, \lambda_{m}=0$ and $\Phi=0$ ), we have that $u_{0}$ is one-homogeneous.

Definition 5.39. We define $d^{*}$ as the smallest dimension which admits one-homogeneous global minimizers of the one-phase Alt-Caffarelli problem with (isolated) singularity in zero.

By [31] and [22] we know that $d^{*} \in\{5,6,7\}$. Weiss was first to prove that the monotonicity formula implies the dimension estimate

$$
\operatorname{dim}_{\mathcal{H}} \operatorname{Sing}\left(\partial \Omega_{u}\right):=\inf \left\{\alpha \geq 0: \mathcal{H}^{\alpha}\left(\operatorname{Sing}\left(\partial \Omega_{u}\right)\right)=0\right\} \leq d-d^{*},
$$

for every $d>d^{*}$ (see also [34 for an argument using only the monotonicity of $W$ ). Thus, as a consequence of Lemma 5.37, Lemma 5.38 and the results from [40] and [34, we get 
Proposition 5.40 (On the dimension of the singular set). Let $u$ be a solution of (5.1) in the bounded open set $D \subset \mathbb{R}^{d}$. Then

- $\operatorname{Sing}\left(\partial \Omega_{u}\right)=\emptyset$ if $d<d^{*}$;

- $\operatorname{Sing}\left(\partial \Omega_{u}\right)$ is a discrete (locally finite) set if $d=d^{*}$;

- $\operatorname{dim}_{\mathcal{H}} \operatorname{Sing}\left(\partial \Omega_{u}\right)<d-d^{*}$ if $d>d^{*}$.

Proof. The proof of this proposition is standard, once we have the monotonicity of $W$ (Lemma 5.37) and the consequent homogeneity of the blow-up limits (Lemma 5.38). We refer to [40, Section 4] and [34, Section 5.5].

Remark 5.41. Recently, using the innovative approach of Naber and Valtorta [36], Edelen and Engelstein 23. showed the monotonicity formula of Weiss can be used to obtain the (local) estimate $\mathcal{H}^{d-d^{*}}\left(\operatorname{Sing}\left(\partial \Omega_{u}\right)\right)<\infty$, which in particular implies that $\operatorname{dim}_{\mathcal{H}} \operatorname{Sing}\left(\partial \Omega_{u}\right)<d-d^{*}$.

\section{Appendix A. Extremality Conditions and LeBesgue Density}

In this section we prove Proposition A.1, which we use in Proposition 5.12 to show that the Lagrange multiplier $\Lambda_{u}$ is strictly positive, but the result is of independent interest. For instance, it applies to optimal partition problems (see, for example, [20] and [17]). We first show that a function which is critical for the functional

$$
J(u):=\int_{D}|\nabla u|^{2} e^{-\Phi} d x-\lambda \int_{D} u^{2} e^{-\Phi} d x,
$$

with respect to internal variations that is

$$
\delta J(u)[\xi]:=\lim _{t \rightarrow 0} J(u(x+t \xi(x)))=0 \quad \text { for every vector field } \quad \xi \in C_{c}^{\infty}\left(D ; \mathbb{R}^{d}\right),
$$

satisfies a monotonicity formula for the associated Almgren frequency function $N(r)$. Now, by the argument of Garofalo and Lin (see [26]) the monotonicity of the frequency function implies that $u$ cannot decay too fast around the free boundary points. If, in addition, $u$ is a solution of $-\operatorname{div}\left(e^{-\Phi} \nabla u\right)=\lambda u e^{-\Phi}$ on the positivity set $\Omega_{u}=\{u>0\}$, we can use a Caccioppoli inequality to show that if the Lebesgue density of $\Omega_{u}$ is too small, then the decay of $u$ on the balls of radius $r$ should be very fast. This, in combination with the monotonicity of the Almgren's frequency function, shows that the Lebesgue density of $\Omega_{u}$ should be bounded from below everywhere (and not only on the boundary of $\Omega_{u}$ ). In particular, there cannot be points of zero Lebesgue density for $\Omega_{u}$ in $D$.

Proposition A.1. Let $D \subset \mathbb{R}^{d}$ be a bounded open set and $\Phi \in W^{1, \infty}(D)$. Suppose that $\lambda \geq 0$ and $u \in H^{1}(D)$ is a nonnegative (non-identically-zero) function such that

(a) $u$ is a solution of the equation

$$
-\operatorname{div}\left(e^{-\Phi} \nabla u\right)=\lambda e^{-\Phi} u \quad \text { in } \quad \Omega_{u}=\{u>0\} ;
$$

(b) $u$ satisfies the extremality condition

$$
\delta J(u)[\xi]=0 \quad \text { for every } \quad \xi \in C_{c}^{\infty}\left(D ; \mathbb{R}^{d}\right),
$$

where $J$ is given by (A.1) and its first variation in the direction $\xi$ is given by

$$
\delta J(u)[\xi]:=\int_{D}\left[2 D \xi(\nabla u) \cdot \nabla u+\left(|\nabla u|^{2}-\lambda u^{2}\right)(\nabla \Phi \cdot \xi-\operatorname{div} \xi)\right] e^{-\Phi} d x .
$$

Then, $\left|D \backslash \Omega_{u}\right|=0$. 
A.1. Reduction to the case $\lambda=0$. In this section we will show that it is sufficient to prove Proposition A.1 for $\lambda=0$. The general case will then follow by an elementary substitution argument. In the next lemma we deal with the first variation of the functional $J$.

Lemma A.2. Suppose that $D \subset \mathbb{R}^{d}$ is a bounded open set, $a: D \rightarrow \mathbb{R}$ is a given Lipschitz function such that $0<\varepsilon \leq a \leq \varepsilon^{-1}$ on $D$. Let $\lambda>0$ and let $\varphi \in H^{2}(D)$ be such that

$$
-\operatorname{div}(a \nabla \varphi)=\lambda a \varphi \quad \text { in } \quad D, \quad \varphi \geq \varepsilon>0 \quad \text { on } \quad D .
$$

For any $u \in H^{1}(D)$, we set $\tilde{a}(x):=\varphi^{2}(x) a(x), \tilde{u}:=u / \varphi$,

$$
\begin{gathered}
J(u):=\int_{D}\left(|\nabla u|^{2}-\lambda u^{2}\right) a(x) d x \quad \text { and } \quad \tilde{J}(u):=\int_{D}|\nabla u|^{2} \tilde{a}(x) d x, \\
\delta J(u)[\xi]:=\int_{D}\left[2 a D \xi(\nabla u) \cdot \nabla u-\left(|\nabla u|^{2}-\lambda u^{2}\right) \operatorname{div}(a \xi)\right] d x, \\
\delta \tilde{J}(u)[\xi]:=\int_{D}\left[2 \tilde{a} D \xi(\nabla u) \cdot \nabla u-|\nabla u|^{2} \operatorname{div}(\tilde{a} \xi)\right] d x \quad \text { for any } \xi \in C_{c}^{\infty}\left(D ; \mathbb{R}^{d}\right) .
\end{gathered}
$$

Then, for every $u \in H^{1}(D)$ and every $\xi \in C_{c}^{\infty}\left(D ; \mathbb{R}^{d}\right)$, we have

$$
\delta \tilde{J}(\tilde{u})[\xi]=\delta J(u)[\xi]-2 \int_{D} \nabla(u \xi \cdot \nabla(\ln \varphi)) \cdot \nabla u a d x+2 \int_{D}(u \xi \cdot \nabla(\ln \varphi)) \lambda a u d x .
$$

Proof. Notice that we may assume $u \in C^{\infty}(D)$. First we notice that an integration by parts gives

$$
\begin{aligned}
\delta \tilde{J}(\tilde{u})[\xi] & =\int_{D} 2 \partial_{i} \xi_{j} \partial_{i} \tilde{u} \partial_{j} \tilde{u} \tilde{a} d x-\int_{D}|\nabla \tilde{u}|^{2} \operatorname{div}(\tilde{a} \xi) d x \\
& =-\int_{D} 2 \xi_{j} \partial_{i}\left(\tilde{a} \partial_{i} \tilde{u}\right) \partial_{j} \tilde{u} d x-\int_{D} 2 \xi_{j} \partial_{i} \tilde{u} \partial_{i j} \tilde{u} \tilde{a} d x-\int_{D}|\nabla \tilde{u}|^{2} \operatorname{div}(\tilde{a} \xi) d x \\
& =-\int_{D} 2 \xi_{j} \partial_{i}\left(\tilde{a} \partial_{i} \tilde{u}\right) \partial_{j} \tilde{u} d x-\int_{D} \operatorname{div}\left(\tilde{a}|\nabla \tilde{u}|^{2} \xi\right) d x=-\int_{D} 2 \xi_{j} \partial_{i}\left(\tilde{a} \partial_{i} \tilde{u}\right) \partial_{j} \tilde{u} d x \\
& =-\int_{D} 2(\xi \cdot \nabla \tilde{u}) \operatorname{div}(\tilde{a} \nabla \tilde{u}) d x .
\end{aligned}
$$

and, analogously,

$$
\delta J(u)[\xi]=-\int_{D} 2(\xi \cdot \nabla u) \operatorname{div}(a \nabla u) d x+\lambda \int_{D} u^{2} \operatorname{div}(a \xi) d x .
$$

Now, since

$$
\operatorname{div}(\tilde{a} \nabla \tilde{u})=\operatorname{div}(a(\varphi \nabla u-u \nabla \varphi))=\varphi \operatorname{div}(a \nabla u)-u \operatorname{div}(a \nabla \varphi)=\varphi(\operatorname{div}(a \nabla u)+\lambda a u),
$$

we get

$$
\begin{aligned}
\delta \tilde{J}(\tilde{u})[\xi] & =-2 \int_{D} \xi \cdot\left(\nabla u-\frac{u}{\varphi} \nabla \varphi\right)(\operatorname{div}(a \nabla u)+\lambda a u) d x \\
& =2 \int_{D} \xi \cdot \nabla \varphi \frac{u}{\varphi}(\operatorname{div}(a \nabla u)+\lambda a u) d x-2 \int_{D}(\xi \cdot \nabla u)(\operatorname{div}(a \nabla u)+\lambda a u) d x \\
& =-2 \int_{D} \nabla\left(\frac{\xi \cdot \nabla \varphi}{\varphi} u\right) \cdot \nabla u a d x+2 \int_{D}\left(\frac{\xi \cdot \nabla \varphi}{\varphi} u\right) \lambda a u d x+\delta J(u)[\xi],
\end{aligned}
$$

which is precisely A.4.

Let now $D \subset \mathbb{R}^{d}$ and $u \in H^{1}(D)$ be as in Proposition A.1 for some $\lambda>0$. In order to prove that $\left|D \backslash \Omega_{u}\right|=0$, it is sufficient to prove that $\left|(D \cap B) \backslash \Omega_{u}\right|=0$ for any (small) ball $B \subset D$. Let now $x_{0} \in D$ and let $R>0$ be such that $\lambda_{1}\left(B_{R}\left(x_{0}\right), \nabla \Phi\right)=\lambda$. Such a radius exists, since the map $f(r):=\lambda_{1}\left(B_{r}\left(x_{0}\right), \nabla \Phi\right)$ is continuous, $f(0)=+\infty$ and $f(+\infty)=0$. Notice also that we may assume $\Phi$ to be defined on the entire space $\mathbb{R}^{d}$. Let $\varphi$ be the first eigenfunction on $B_{R}\left(x_{0}\right)$ 
and let $r=R / 2$. Then, we can apply Lemma A.2 in the set $D \cap B_{r}\left(x_{0}\right)$ with $a=e^{-\Phi}$. Moreover, since $u$ satisfies (A.2), we get that

$$
\delta \tilde{J}(\tilde{u})[\xi]=\delta J(u)[\xi]=0, \quad \text { for every } \xi \in C_{c}^{\infty}\left(D \cap B_{r}\left(x_{0}\right) ; \mathbb{R}^{d}\right),
$$

which proves that $\tilde{u}=u / \varphi$ satisfies hypothesis (b) for $\lambda=0$. Finally, in order to prove that $\tilde{u}$ satisfies hypothesis (a), we notice that on $\Omega_{u}=\Omega_{\tilde{u}}$ we have (in a weak sense)

$$
\operatorname{div}(\tilde{a} \nabla \tilde{u})=\varphi \operatorname{div}(a \nabla u)-u \operatorname{div}(a \nabla \varphi)=\varphi(\operatorname{div}(a \nabla u)+\lambda a u)=0 .
$$

A.2. Proof of Proposition A.1 in the case $\lambda=0$. Let $\lambda=0$. Then we have

$$
\begin{gathered}
J(u):=\int_{D}|\nabla u|^{2} e^{-\Phi} d x \\
\delta J(u)[\xi]:=\int_{D}\left[2 D \xi(\nabla u) \cdot \nabla u+|\nabla u|^{2}(\nabla \Phi \cdot \xi-\operatorname{div} \xi)\right] e^{-\Phi} d x .
\end{gathered}
$$

Let $x_{0}=0 \in D$ and $\tau=\|\nabla \Phi\|_{L^{\infty}(D)}$. We set

$$
H(r):=\int_{\partial B_{r}} u^{2} e^{-\Phi} d \mathcal{H}^{d-1}, \quad D(r):=\int_{B_{r}}|\nabla u|^{2} e^{-\Phi} d x \quad \text { and } \quad N(r):=\frac{r D(r)}{H(r)} .
$$

Step 1. Derivative of $H$. We calculate

$$
\begin{aligned}
H^{\prime}(r) & =\frac{d-1}{r} H(r)+r^{d-1} \frac{d}{d r} \int_{\partial B_{1}} u^{2}(r x) e^{-\Phi(r x)} d \mathcal{H}^{d-1}(x) \\
& =\frac{d-1}{r} H(r)+2 \int_{\partial B_{r}} u \frac{\partial u}{\partial n} e^{-\Phi} d \mathcal{H}^{d-1}-\int_{\partial B_{r}} u^{2}(n \cdot \nabla \Phi) e^{-\Phi} d \mathcal{H}^{d-1} \\
& =\frac{d-1}{r} H(r)+2 \int_{B_{r}}|\nabla u|^{2} e^{-\Phi} d x-\int_{\partial B_{r}} u^{2}(n \cdot \nabla \Phi) e^{-\Phi} d \mathcal{H}^{d-1}
\end{aligned}
$$

which we rewrite as

$$
H^{\prime}(r)=\frac{d-1}{r} H(r)+2 D(r)-H_{\Phi}(r) .
$$

where we have set

$$
H_{\Phi}(r):=\int_{\partial B_{r}} u^{2}(n \cdot \nabla \Phi) e^{-\Phi} d \mathcal{H}^{d-1} \quad \text { and } \quad\left|H_{\Phi}(r)\right| \leq \tau H(r) .
$$

Step 2. Equidistribution of the energy. Let $\phi_{\varepsilon}$ be a radially decreasing function such that $0 \leq \phi_{\varepsilon} \leq$ 1 on $B_{r}, \phi_{\varepsilon}=1$ on $B_{r(1-\varepsilon)}, \phi_{\varepsilon}=0$ on $\partial B_{r}$ and $\left|\nabla \phi_{\varepsilon}\right| \leq C(r \varepsilon)^{-1}$. The vector field $\xi(x):=x \phi_{\varepsilon}(x)$ satisfies $\operatorname{div} \xi(x)=d \phi_{\varepsilon}(x)+x \cdot \nabla \phi_{\varepsilon}$ and $\partial_{i} \xi_{j}=\delta_{i j} \phi_{\varepsilon}(x)+x_{j} \partial_{i} \phi_{\varepsilon}(x)$. Since $\lambda=0$ we have

$$
\begin{aligned}
\delta J(u)[\xi]= & \int_{D}\left[2 D \xi(\nabla u) \cdot \nabla u+|\nabla u|^{2}(\nabla \Phi \cdot \xi-\operatorname{div} \xi)\right] e^{-\Phi} d x \\
= & \int_{D}\left[2|\nabla u|^{2} \phi_{\varepsilon}+2(x \cdot \nabla u)\left(\nabla \phi_{\varepsilon} \cdot \nabla u\right)-|\nabla u|^{2}\left(d \phi_{\varepsilon}(x)+x \cdot \nabla \phi_{\varepsilon}\right)\right] e^{-\Phi} d x \\
& +\int_{D}|\nabla u|^{2}(\nabla \Phi \cdot x) \phi_{\varepsilon} e^{-\Phi} d x,
\end{aligned}
$$

and passing to the limit as $\varepsilon \rightarrow 0$, rearraging the terms and using the property (b), we get

$$
\begin{aligned}
0=- & (d-2) \int_{B_{r}}|\nabla u|^{2} e^{-\Phi} d x+r \int_{\partial B_{r}}|\nabla u|^{2} e^{-\Phi} d \mathcal{H}^{d-1} \\
& -2 r \int_{\partial B_{r}}\left(\frac{\partial u}{\partial n}\right)^{2} e^{-\Phi} d \mathcal{H}^{d-1}+\int_{B_{r}}|\nabla u|^{2}(\nabla \Phi \cdot x) e^{-\Phi} d x,
\end{aligned}
$$

which we rewrite as

$$
-(d-2) D(r)+r D^{\prime}(r)=2 r \int_{\partial B_{r}}\left(\frac{\partial u}{\partial n}\right)^{2} e^{-\Phi} d \mathcal{H}^{d-1}-r D_{\Phi}(r),
$$


where

$$
D_{\Phi}(r):=\frac{1}{r} \int_{B_{r}}|\nabla u|^{2}(\nabla \Phi \cdot x) e^{-\Phi} d x \quad \text { and } \quad\left|D_{\Phi}(r)\right| \leq \tau D(r) .
$$

Step 3. The derivative of $N$. We notice that $N(r)$ is only defined for $r$ such that $H(r)>0$. In what follows we fix $r_{0}>0$ such that $B_{r_{0}}\left(x_{0}\right) \subset D$ and $H\left(r_{0}\right)>0$. Since $u \in H^{1}(D)$, there is an interval $(a, b) \ni r_{0}$, on which $H>0$.

$$
\begin{aligned}
N^{\prime}(r) & =\frac{D(r) H(r)+r D^{\prime}(r) H(r)-r D(r) H^{\prime}(r)}{H^{2}(r)} \\
& =\frac{D(r) H(r)+r D^{\prime}(r) H(r)-r D(r)\left(\frac{d-1}{r} H(r)+2 D(r)-H_{\Phi}(r)\right)}{H^{2}(r)} \\
& =\frac{-(d-2) D(r) H(r)+r D^{\prime}(r) H(r)-2 r D^{2}(r)+r D(r) H_{\Phi}(r)}{H^{2}(r)} \\
& =\frac{2 r}{H^{2}(r)}\left(H(r) \int_{\partial B_{r}}\left(\frac{\partial u}{\partial n}\right)^{2} e^{-\Phi} d \mathcal{H}^{d-1}-D^{2}(r)\right)+\frac{r\left(D(r) H_{\Phi}(r)-D_{\Phi}(r) H(r)\right)}{H^{2}(r)}
\end{aligned}
$$

Now we notice that, since $u$ solves (A.2) on $\Omega_{u}$, we have

$$
D(r)=\int_{B_{r}}|\nabla u|^{2} e^{-\Phi} d x=\int_{\partial B_{r}} u \frac{\partial u}{\partial n} e^{-\Phi} d \mathcal{H}^{d-1},
$$

and so, by the Cauchy-Schwarz inequality and (A.8) we obtain

$$
N^{\prime}(r) \geq \frac{r\left(D(r) H_{\Phi}(r)-D_{\Phi}(r) H(r)\right)}{H^{2}(r)} \geq-2 \tau N(r) .
$$

Step 4. A bound on $N(r)$. Using the estimate A.9 from the previous step we get that the function $r \mapsto e^{2 \tau r} N(r)$ is non-decreasing in $r$ and so

$$
N(r) \leq e^{2 \tau\left(r_{0}-r\right)} N\left(r_{0}\right) \leq e^{2 \tau r_{0}} N\left(r_{0}\right) \text { for every } a<r \leq r_{0} .
$$

Step 5. Strict positivity and doubling inequality for $H(r)$. By the step 4 we have

$$
\frac{d}{d r}\left[\log \left(\frac{H(r)}{r^{d-1}}\right)\right]=2 \frac{N(r)}{r}-\frac{H_{\Phi}(r)}{H(r)} \leq \frac{2 e^{2 \tau r_{0}} N\left(r_{0}\right)}{r}+\tau,
$$

and integrating we get

$$
\log \left(\frac{H\left(r_{0}\right)}{r_{0}^{d-1}}\right)-\log \left(\frac{H(r)}{r^{d-1}}\right) \leq \log \left(\frac{r_{0}}{r}\right) 2 e^{2 \tau r_{0}} N\left(r_{0}\right)+\tau r_{0}, \quad \text { for every } \quad a<r \leq r_{0} .
$$

In particular, $H>0$ on every interval $\left[\varepsilon r_{0}, r_{0}\right]$ and so, $H>0$ on $\left(0, r_{0}\right]$ and we might take $a=0$. Moreover, integrating once again the inequality (A.10) from $r<r_{0} / 2$ to $2 r$, we get

$$
\log \left(\frac{H(2 r)}{H(r)}\right) \leq\left((d-1) \log 2+\tau r_{0}\right)+2 \log 2 e^{2 \tau r_{0}} N\left(r_{0}\right) \quad \text { for every } \quad 0<r \leq \frac{r_{0}}{2} .
$$

Taking $r_{0} \leq 1$, there is a constant $C$, depending only on $d$ and $\tau$, such that

$$
H(2 r) \leq C \exp \left(C N\left(r_{0}\right)\right) H(r) \quad \text { for every } \quad 0<r \leq \frac{r_{0}}{2} .
$$

Integrating once more in $r$ we get

$$
\int_{B_{2 r}} u^{2} e^{-\Phi} d x \leq C \exp \left(C N\left(r_{0}\right)\right) \int_{B_{r}} u^{2} e^{-\Phi} d x \quad \text { for every } \quad 0<r \leq \frac{r_{0}}{2} .
$$

Step 6. Caccioppoli inequality and conclusion. Let $r \in\left(0, r_{0} / 2\right]$ and let $\phi \in C_{0}^{\infty}\left(B_{2 r}\right)$ be such that $\phi=1$ in $B_{r}, \phi=0$ on $\partial B_{2 r}, 0 \leq \phi \leq 1$ and $|\nabla \phi| \leq 2 / r$ on $B_{2 r} \backslash B_{r}$. Using the fact that $u$ is a 
solution of $-\operatorname{div}\left(e^{-\Phi} \nabla u\right)=0$ in $\Omega_{u}$, we get the following Caccioppoli inequality:

$$
\begin{aligned}
\int_{B_{r}}|\nabla u|^{2} e^{-\Phi} d x & \leq \int_{B_{2 r}}|\nabla(u \phi)|^{2} e^{-\Phi} d x=\int_{B_{2 r}}\left(u^{2}|\nabla \phi|^{2}+\nabla u \cdot \nabla\left(u \phi^{2}\right)\right) e^{-\Phi} d x \\
& \left.=\int_{B_{2 r}} u^{2}|\nabla \phi|^{2} e^{-\Phi} d x-\int_{B_{2 r}} u \phi^{2} \operatorname{div}\left(e^{-\Phi} \nabla u\right)\right) d x=\int_{B_{2 r}} u^{2}|\nabla \phi|^{2} e^{-\Phi} d x \\
& \leq \frac{4}{r^{2}} \int_{B_{2 r}} u^{2} e^{-\Phi} d x
\end{aligned}
$$

On the other hand, there are dimensional constants $C_{d}$ and $\varepsilon_{d}>0$ such that, if $\left|\Omega_{u} \cap B_{r}\right| \leq \varepsilon_{d}\left|B_{r}\right|$, then the following inequality does hold (see [15, Lemma 4.4])

$$
\int_{B_{r}} u^{2} d x \leq C_{d} r^{2}\left(\frac{\left|\Omega_{u} \cap B_{r}\right|}{\left|B_{r}\right|}\right)^{2 / d} \int_{B_{r}}|\nabla u|^{2} d x,
$$

which, taking $C:=C_{d} \exp (\max \Phi-\min \Phi)$, implies

$$
\int_{B_{r}} u^{2} e^{-\Phi} d x \leq C r^{2}\left(\frac{\left|\Omega_{u} \cap B_{r}\right|}{\left|B_{r}\right|}\right)^{2 / d} \int_{B_{r}}|\nabla u|^{2} e^{-\Phi} d x .
$$

This, together with (A.13) and the doubling inequality (A.12), gives that there are constants $C_{1}$ and $C_{2}$, depending only on $d$ and $\tau$ such that

$$
\min \left\{\varepsilon_{d}, C_{1} \exp \left(-C_{2} N\left(r_{0}\right)\right)\right\} \leq \frac{\left|\Omega_{u} \cap B_{r}\right|}{\left|B_{r}\right|} \quad \text { for every } \quad 0<r \leq \frac{r_{0}}{2},
$$

where to be precise we recall that we assumed $r_{0} \leq 1$. In particular, we have a lower density bound for $\Omega_{u}$ at every point of $D$, which implies that $\left|D \backslash \Omega_{u}\right|=0$ and concludes the proof.

Acknowledgments. The authors have been partially supported by Agence Nationale de la Recherche (ANR) by the projects GeoSpec (LabEx PERSYVAL-Lab, ANR-11-LABX-0025-01). The third author was also partially supported by the project ANR CoMeDiC (ANR-15-CE400006).

\section{REFERENCES}

[1] N. Aguilera, H. W. Alt, and L. A. Caffarelli. An optimization problem with volume constraint. SIAM J. Control Optim., 24:191-198, 1986.

[2] H. W. Alt and L. A. Caffarelli. Existence and regularity for a minimum problem with free boundary. J. Reine Angew. Math., 325:105-144, 1981.

[3] H. W. Alt, L. A. Caffarelli, and A. Friedman. Variational problems with two phases and their free boundary. Trans. Am. Math. Soc., 282:431-461, 1984.

[4] H. Berestycki, L. Nirenberg, and S. R. S. Varadhan. The principal eigenvalue and maximum principle for second-order elliptic operators in general domains. Commun. Pure Appl. Math., 47(1):47-92, 1994.

[5] T. Briançon, M. Hayouni, and M. Pierre. Lipschitz continuity of state functions in some optimal shaping. Calc. Var. Partial Differ. Equ., 23(1):13-32, 2005.

[6] T. Briançon and J. Lamboley. Regularity of the optimal shape for the first eigenvalue of the Laplacian with volume and inclusion constraints. Ann. Inst. Henri Poincaré, Anal. Non Linéaire, 26(4):1149-1163, 2009.

[7] T. Briancon. Regularity of optimal shapes for the Dirichlet's energy with volume constraint. ESAIM, Control Optim. Calc. Var., 10:99-122, 2004.

[8] D. Bucur. Minimization of the $k$-th eigenvalue of the Dirichlet Laplacian. Arch. Ration. Mech. Anal., 206(3):1073-1083, 2012.

[9] D. Bucur and G. Buttazzo. Variational methods in shape optimization problems., volume 65. Basel: Birkhäuser, 2005.

[10] D. Bucur, D. Mazzoleni, A. Pratelli, and B. Velichkov. Lipschitz regularity of the eigenfunctions on optimal domains. Arch. Ration. Mech. Anal., 216(1):117-151, 2015.

[11] D. Bucur and B. Velichkov. Multiphase shape optimization problems. SIAM J. Control Optim., 52(6):35563591, 2015.

[12] G. Buttazzo. Spectral optimization problems. Rev. Mat. Complut., 24(2):277-322, 2011.

[13] G. Buttazzo and G. Dal Maso. An existence result for a class of shape optimization problems. Arch. Ration. Mech. Anal., 122(2):183-195, 1993. 
[14] G. Buttazzo and B. Velichkov. The spectral drop problem. In Recent advances in partial differential equations and applications. International conference in honor of Hugo Beirão de Veiga's 70th birthday, Levico Terme, Italy, February 17-21, 2014. Proceedings, pages 111-135. Providence, RI: American Mathematical Society (AMS), 2016.

[15] G. Buttazzo and B. Velichkov. A shape optimal control problem with changing sign data. SIAM J. Math. Anal., 50(3):2608-2627, 2018.

[16] L. A. Caffarelli, D. Jerison, and C. E. Kenig. Global energy minimizers for free boundary problems and full regularity in three dimensions. In Noncompact problems at the intersection of geometry, analysis, and topology. Proceedings of the conference on noncompact variational problems and general relativity held in honor of Haim Brezis and Felix Browder at Rutgers University, New Brunswick, NJ, USA, October 14-18, 2001, pages 83-97. Providence, RI: American Mathematical Society (AMS), 2004.

[17] L. A. Caffarelli and F.-H. Lin. Singularly perturbed elliptic systems and multi-valued harmonic functions with free boundaries. J. Am. Math. Soc., 21(3):847-862, 2008.

[18] H. Chang-Lara and O. Savin. Boundary regularity for the free boundary in the one-phase problem. arXiv preprint arXiv:1709.03371, 2017.

[19] D. Cioranescu and F. Murat. Un terme etrange venu d'ailleurs. Nonlinear partial differential equations and their applications, Coll. de France Semin., Vol. II, Res. Notes Math. 60, 98-138 (1982)., 1982.

[20] M. Conti, S. Terracini, and G. Verzini. An optimal partition problem related to nonlinear eigenvalues. J. Funct. Anal., 198(1):160-196, 2003.

[21] D. De Silva. Free boundary regularity for a problem with right hand side. Interfaces Free Bound., 13(2):223238, 2011.

[22] D. De Silva and D. Jerison. A singular energy minimizing free boundary. J. Reine Angew. Math., 635:1-21, 2009.

[23] N. Edelen and M. Engelstein. Quantitative stratification for some free-boundary problems. arXiv preprint arXiv:1702.04325, 2017.

[24] L. C. Evans. Partial differential equations., volume 19. Providence, RI: American Mathematical Society, 1998.

[25] L. C. Evans and R. F. Gariepy. Measure theory and fine properties of functions. 2nd revised ed. Boca Raton, FL: CRC Press, 2nd revised ed. edition, 2015.

[26] N. Garofalo and F.-H. Lin. Monotonicity properties of variational integrals, $A_{p}$ weights and unique continuation. Indiana Univ. Math. J., 35:245-268, 1986.

[27] D. Gilbarg and N. S. Trudinger. Elliptic partial differential equations of second order. Reprint of the 1998 ed. Berlin: Springer, reprint of the 1998 ed. edition, 2001.

[28] F. Hamel, N. Nadirashvili, and E. Russ. A faber-krahn inequality with drift. arXiv preprint math/0607585, 2006.

[29] F. Hamel, N. Nadirashvili, and E. Russ. Rearrangement inequalities and applications to isoperimetric problems for eigenvalues. Ann. Math. (2), 174(2):647-755, 2011.

[30] A. Henrot and M. Pierre. Variation et optimisation de formes. Une analyse géométrique., volume 48. Berlin: Springer, 2005.

[31] D. Jerison and O. Savin. Some remarks on stability of cones for the one-phase free boundary problem. Geom. Funct. Anal., 25(4):1240-1257, 2015.

[32] T. Kato. Perturbation theory for linear operators. Reprint of the corr. print. of the 2nd ed. 1980. Berlin: Springer-Verlag, reprint of the corr. print. of the 2nd ed. 1980 edition, 1995.

[33] D. Kinderlehrer and L. Nirenberg. Regularity in free boundary problems. Ann. Sc. Norm. Super. Pisa, Cl. Sci., IV. Ser., 4:373-391, 1977.

[34] D. Mazzoleni, S. Terracini, and B. Velichkov. Regularity of the optimal sets for some spectral functionals. Geom. Funct. Anal., 27(2):373-426, 2017.

[35] D. Mazzoleni, S. Terracini, and B. Velichkov. Regularity of the free boundary for the vectorial bernoulli problem. arXiv preprint arXiv:1804.09243, 2018.

[36] A. Naber and D. Valtorta. Rectifiable-Reifenberg and the regularity of stationary and minimizing harmonic maps. Ann. Math. (2), 185(1):131-227, 2017.

[37] L. Spolaor, B. Trey, and B. Velichkov. Free boundary regularity for a multiphase shape optimization problem. arXiv preprint arXiv:1810.06963, 2018.

[38] L. Spolaor and B. Velichkov. An epiperimetric inequality for the regularity of some free boundary problems: the 2-dimensional case. arXiv preprint arXiv:1612.01623, 2016.

[39] B. Velichkov. Existence and regularity results for some shape optimization problems. Pisa: Edizioni della Normale; Pisa: Scuola Normale Superiore (Diss. 2013), 2015.

[40] G. S. Weiss. Partial regularity for a minimum problem with free boundary. J. Geom. Anal., 9(2):317-326, 1999. 
EMmanuel Russ:

Université Grenoble Alpes, CNRS UMR 5582, Institut Fourier

100 Rue des Mathématiques, F-38610 Gières, France

E-mail address: emmanuel.russ@univ-grenoble-alpes.fr

BAPtiste TREY:

Université Grenoble Alpes, CNRS UMr 5582, Institut Fourier

100 rue des Mathématiques, F-38610 Gières, France

E-mail address: baptiste.trey@etu.univ-grenoble-alpes.fr

BOZHIDAR VELICHKOV:

Université Grenoble Alpes, CNRS UMR 5224

700 avenue Centrale, F-38401 Domaine Universitaire de Saint-Martin-D'Hères, France

E-mail address: bozhidar.velichkov@univ-grenoble-alpes.fr 